Contact information:

Jord Prangsma

Jordprangsma at gmail.com 
LOCAL AND DYNAMIC PROPERTIES OF LIGHT INTERACTING WITH SUBWAVELENGTH HOLES 
Samenstelling van de promotiecommissie:

prof. dr. L. Kuipers (promotor) Universiteit Twente prof. dr. K. J. Boller Universiteit Twente prof. dr. F. J. Garcia-Vidal Universidad Autónoma de Madrid prof. dr. J. L. Herek Universiteit Twente prof. dr. ir. H. Hilgenkamp Universiteit Twente prof. dr. P. C. M. Planken Technische Universiteit Delft

This research was supported by NanoNed, a national nanotechnology program coordinated by the Dutch Ministry of Economic Affairs (projectnumber 6643) and is part of the research program of the "Stichting Fundamenteel Onderzoek der Materie" (FOM), which is financially supported by the

"Nederlandse Organisatie voor Wetenschappelijk Onderzoek" (NWO).

This work was carried out at:

NanoOptics Group, FOM-Institute for Atomic and Molecular Physics (AMOLF) Science Park 113, 1098 XG Amsterdam, The Netherlands, where a limited number of copies of this thesis is available.

ISBN: 978-90-365-2809-2

Printed by Ipskamp, Enschede, The Netherlands. 


\section{LOCAL AND DYNAMIC PROPERTIES OF LIGHT INTERACTING WITH SUBWAVELENGTH HOLES}

\section{PROEFSCHRIFT}

ter verkrijging van

de graad van doctor aan de Universiteit Twente,

op gezag van de rector magnificus,

prof. dr. H. Brinksma,

volgens besluit van het College voor Promoties

in het openbaar te verdedigen

op vrijdag 8 mei 2009 om 13:15 uur

door

Jord Cornelis Prangsma

geboren op 20 mei 1979

te Apeldoorn 
Dit proefschrift is goedgekeurd door: prof. dr. L. (Kobus) Kuipers 


\section{Contents}

1 Introduction $\mathbf{5}$

1.1 Outline of this thesis ............... 5

2 Nanoplasmonics $\quad 7$

2.1 Introduction . . . . . . . . . . . . . . 7

2.2 The optical properties of gold . . . . . . . . . . . 8

2.3 Surface plasmon polaritons . . . . . . . . . . . . 9

2.4 Localized surface plasmons . . . . . . . . . . . . . . 12

2.5 Surface waves . . . . . . . . . . . . . . . . . . . . . 13

2.6 Extraordinary transmission . . . . . . . . . . . . 15

2.6.1 Single subwavelength holes . . . . . . . . . . 15

2.6.2 Arrays of subwavelength holes . . . . . . . . . . . 19

3 Group delay through subwavelength hole arrays $\quad 21$

3.1 Introduction . . . . . . . . . . . . . . . . 21

3.2 Subwavelength hole array structures . . . . . . . . . 22

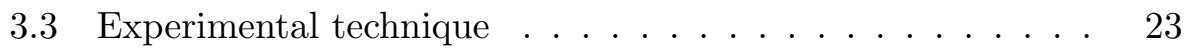

3.4 Experimental results . . . . . . . . . . . . . . 25

3.5 Finite difference time domain calculations . . . . . . . 26

3.6 Second harmonic generation . . . . . . . . . . . . 28

3.7 The role of attenuation . . . . . . . . . . . . . . 30

3.8 Conclusion . . . . . . . . . . . . . . . . 30

4 Transition radiation $\quad 33$

4.1 The field of a moving point charge . . . . . . . . . . 34

4.2 The field of the transition radiation . . . . . . . . . . 38

4.2.1 Dispersion diagrams for the transition radiation . . . 40 
4.2.2 The expression for transition radiation . . . . . . . 42

4.3 Transition radiation in real space . . . . . . . . . . . . 43

4.3.1 Numerical solution of the Fourier integral . . . . . . 44

4.3.2 Analytical solution of the Fourier integral . . . . . . 45

4.3.3 Surface waves generated by transition radiation . . . 46

4.4 Comparison of the transition radiation with the field of a dipole . . . . . . . . . . . . . . 47

4.5 Transition radiation for nano-optics . . . . . . . . . . 50

5 Transition radiation microscopy 53

5.1 Introduction . . . . . . . . . . . . . . 53

5.2 Principles of operation . . . . . . . . . . . . . . . 54

5.3 Transition radiation microscopy . . . . . . . . . . . . . . 54

5.4 Instrumental information . . . . . . . . . . . . 57

6 Scattering of surface plasmon polaritons on single holes $\quad 59$

6.1 Introduction . . . . . . . . . . . . . . 59

6.2 Transition radiation near a single hole . . . . . . . . . 60

6.3 The effective dipole scattering cross section . . . . . . . . 63

6.4 Discussion . . . . . . . . . . . . . . . 65

7 Local investigation of rectangular holes $\quad 67$

7.1 Rectangular holes . . . . . . . . . . . . . . 68

7.2 Transition radiation microscopy on a single rectangular hole 68

7.3 Systematic investigation of the effect of hole shape . . . . 72

7.4 Interpretation . . . . . . . . . . . . . . 76

8 Polarization-resolved scattering from subwavelength holes $\mathbf{7 9}$

8.1 Introduction . . . . . . . . . . . . . . . 79

8.2 Induced dipole moments in a rectangular hole . . . . . . . . 80

8.3 Polarization selection . . . . . . . . . . . . . 80

8.4 Results . . . . . . . . . . . . . . . . . . . . . . 83

8.5 Conclusion and discussion . . . . . . . . . . . . . 83

9 Scattering from multiple holes $\quad 87$

9.1 Introduction . . . . . . . . . . . . . . . 87

9.2 Results. . . . . . . . . . . . . . . . . . . . . . 88

9.3 Conclusions . . . . . . . . . . . . . . . . . . . . . . . . . 91 
A Group delay through attenuating media 93

A.1 Group velocity in absorbing media . . . . . . . . . . . 94

A.1.1 The Fourier-limited pulse . . . . . . . . . . . 95

A.1.2 The chirped pulse . . . . . . . . . . . 95

A.2 Measurement method for the group delay . . . . . . . . 96

A.2.1 Transfer function . . . . . . . . . . . . 96

A.2.2 Interferometric detection . . . . . . . . . . 97

A.3 Conclusion . . . . . . . . . . . . . . . 100

$\begin{array}{ll}\text { Bibliography } & 101\end{array}$

$\begin{array}{ll}\text { Samenvatting } & 109\end{array}$

$\begin{array}{ll}\text { Dankwoord } & 113\end{array}$ 


\section{Chapter 1}

\section{Introduction}

\subsection{Outline of this thesis}

The field of nano-optics is a flourishing field of research that has grown rapidly during the past 20 years. The goal within the field is to control the flow of light at a scale smaller than its wavelength. Nano-plasmonics is currently the most promising way to realize this miniaturization of optics, as the electromagnetic fields in plasmonic structures are strongly confined to the surface.

This thesis studies one of the most popular structures in nano-plasmonics: A subwavelength hole in a metal film. This geometry has been the subject of many studies since the discovery of extraordinary transmission by Ebbesen and co-workers [1]. This intriguing effect, leading to high transmission through arrays of subwavelength apertures, has greatly contributed to the increasing interest in the field of plasmonics over the past 10 years. Two topics are investigated in this thesis, both with the objective to gain understanding of light interaction with subwavelength holes. One topic concentrates on studying the dynamic properties, while the other focusses on the local optical properties of subwavelength holes. Central to both studies is the role of hole shape. The dynamic properties will be investigated in an experimental study of the propagation of light pulses through subwavelength holes (Chapter 3). We will see how the shape of holes in a subwavelength hole array influences the group delay of light pulses through these structures. The results are linked with nonlinear experiments to investigate the potential of structures of this type to increase light-matter 
interactions. The aim of the second study is to experimentally investigate the local optical properties of single and multiple subwavelength holes in the visible regime. To obtain a high spatial resolution, an electron beam was used to generate light on the sample via a mechanism that is called transition radiation $[2,3,4,5]$. In Chapter 3 the theory necessary to understand this technique is discussed. The experimental setup is described in Chapter 6. The results of transition radiation microscopy measurements on subwavelength holes are discussed in Chapters 6 to 9, focussing respectively on the scattering of surface plasmons on a hole, measurements in the nearfield regime, the effects of polarization, and the interaction of multiple holes. This thesis starts of with an introductory chapter on nano-plasmonics. 


\section{Chapter 2}

\section{Nanoplasmonics}

In this chapter some basic nanoplasmonic concepts, that are used within this thesis, are introduced. We discuss the optical properties of gold and the basic properties of surface plasmon polaritons. Localized plasmons and lossy surface waves are introduced. To sketch the context in which this research has been performed, the extraordinary transmission effect and the transmission through subwavelength holes is reviewed.

\section{$2.1 \quad$ Introduction}

Nano-plasmonics is the field of research that studies the interaction of light with conductors at the nanoscale. The coupling between the electromagnetic field and collective charge oscillations on the interface between a conductor and a dielectric gives rise to a wealth of phenomena that are of interest to both fundamental and applied research. Amongst these phenomena is the beaming of light from a subwavelength aperture; a careful design of concentric surface corrugations around an aperture in a metal layer allows the formation of a light beam with a width that is comparable to the wavelength of the light [6]. Localized plasmon resonances on small metal particles lead to high field enhancements on their surface. Using the resonant localized plasmon field of a bow tie antenna, coherent extremeultraviolet light was created [7]. Nano-plasmonics is at the heart of the current rise of research into metamaterials, enabling exciting physics and applications such as the 'perfect' lens: a lens capable of imaging the complete electromagnetic field of an object [8]. Perhaps the most spectacular 
result within this field of research will be optical cloaking [9]. This science fiction like effect might one day become possible due to nanoplasmonic engineering. It is beyond the scope of this thesis to discuss the wide range of topics that are studied in this field. The aim of this Chapter is to introduce the basic concepts of nano-plasmonics to outline the context within which the research described in this thesis was done. For this reason some basic properties of the dominant material used in the experiments in this thesis - gold - are described. Subsequently, surface plasmon polaritons, localized plasmons and lossy surface waves are introduced. Finally, the extraordinary transmission phenomenon and the optical properties of a hole in a metal layer are discussed.

\subsection{The optical properties of gold}

Throughout this thesis the structures under investigation are fabricated in gold. A practical reason for this is that gold samples retain their good quality for a reasonable amount of time. This is due to the chemical inertness of this noble metal. Additionally, gold is an easily obtainable material that can be evaporated on a surface in a thin smooth layer. A more fundamental reason to use gold for the experiments in this thesis are its optical properties. These properties are best described with the dielectric constant $\varepsilon$, which is a function of the vacuum wavelength. The real and imaginary part of the dielectric constant of gold are plotted in Figure 2.1 as a function of wavelength [10]. These graphs show that the real part of $\varepsilon$ is negative and has much larger values than the imaginary part. This is a characteristic that gold has in common with many metals, and it arises from the contribution of the conduction electrons of the metal to the dielectric constant. The contribution can be neatly described by a Drude model [11, 12], in which the response of the metal to external electromagnetic waves is described as a driven Lorentz oscillator in the limit of no restoring force. This leads to a dielectric constant of the form $\varepsilon=1-\omega_{p}^{2} / \omega^{2}$ with $\omega$ the frequency of the light and $\omega_{p}$ the plasma frequency. In bulk gold the frequency $\omega_{p}$ corresponds to light with a vacuum wavelength of $140 \mathrm{~nm}$. A Drude model was fitted to the measured value of dielectric constant, with the plasma frequency and a damping term as fit parameters (see the dashed curves in Figure 2.1). The model proves to be a good description of the dielectric index above $600 \mathrm{~nm}$. however, at wavelengths below $600 \mathrm{~nm}$ the Drude 

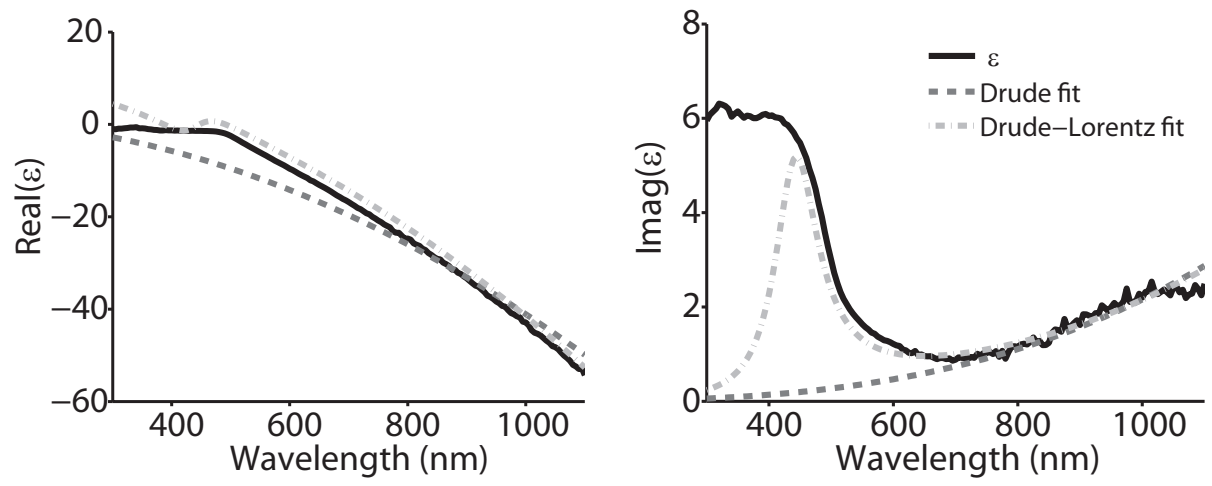

Figure 2.1: Real and imaginary part of dielectric constant of gold with fits of Drude (dashed $\hbar \omega_{p}=8.03 \mathrm{eV} \hbar \Gamma=63.7 \mathrm{meV}$ ) and Drude-Lorentz model (dash-dot $\lambda_{0}=442 \mathrm{~nm}$ ). The value of the dielectric constant used is a measured value, obtained in an ellipsometry measurement.

model is not a suitable model to describe the optical properties of gold. At these high frequencies, the metal starts to behave less and less as a Drude metal because apart from the conduction electrons, interband transitions of the bound valance electrons come into play as well. The contribution of the valance electrons can be modeled with a Lorentz oscillator with a restoring force leading to a real resonance frequency. A model consisting of a combined Drude and Lorentz contribution can be fitted to the measured dielectric constant. The result of this fit is shown as a dash-dotted curve in Figure 2.1.

Summarizing, two regimes can be distinguished in the optical properties of gold within the region 400-1200 nm. Above $600 \mathrm{~nm}$, the free electron contribution dominates the behavior of $\varepsilon$. Here, gold can be considered an ideal metal. Below $600 \mathrm{~nm}$, the bound electrons play the most important role and the optical properties differ distinctively from an ideal meal.

\subsection{Surface plasmon polaritons}

A surface plasmon polariton (SPP) is a surface bound wave that results from the resonant coupling of charge density oscillations of the conduction electrons in a metal with the electromagnetic field. It arises naturally as a 
homogeneous solution of the Maxwell equations on a flat metal-dielectric interface. The electromagnetic field of a SPP propagates over a metal surface as longitudinal surface charge density oscillation and decays in both directions perpendicular to the surface. The dispersion relation of these waves can be derived from boundary conditions for the fields on the surface and has the form [13]

$$
k_{s p}=\frac{\omega}{c} \frac{\varepsilon_{1} \varepsilon_{2}}{\varepsilon_{1}+\varepsilon_{2}},
$$

where $k_{s p}$ is the surface plasmon wavevector, $c$ is the velocity of light in

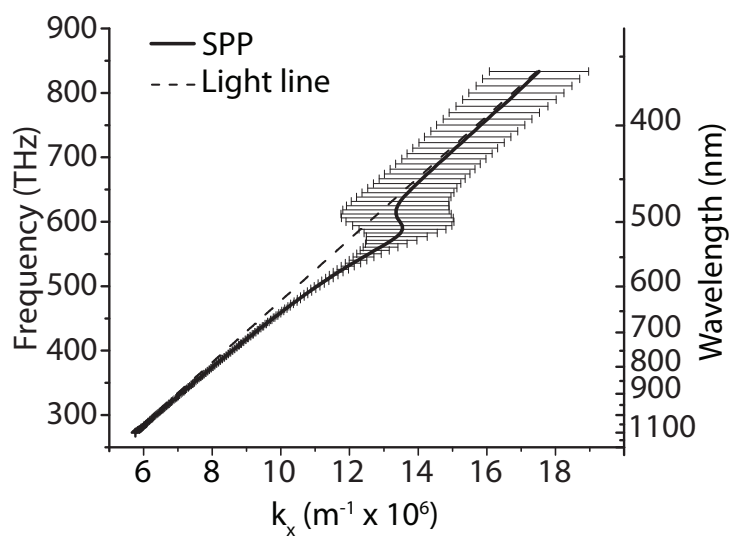

Figure 2.2: The SPP dispersion curve for a gold-air interface. The horizontal bars indicate the imaginary part of the in-plane wavevector. Values in the figure are calculated based on a measured value of the dielectric constant.

vacuum, and $\varepsilon_{1}$ and $\varepsilon_{2}$ are the dielectric constants of the two media. To form a genuine surface bound mode, the dielectric constants of the two materials must satisfy the conditions $\varepsilon_{1} \varepsilon_{2}<0$ and $\varepsilon_{1}+\varepsilon_{2}<0$. These conditions ensure that the wave vector is mainly real in the direction along the surface while $k_{z}$, the wavevector perpendicular to the interface, is mainly imaginary. In Figure 2.2 the dispersion of a SPP on a gold-air interface as calculated with Equation 2.2 is shown. The horizontal bars in the graph are the magnitude of the imaginary part of $k_{s p}$ and indicate the width of the $\mathrm{SPP}$ resonance in wavevector space. Below $600 \mathrm{THz}$ (above $500 \mathrm{~nm}$ ), the dispersion curve lies entirely under the light-line. Since the SPP wavevec- 
tor is too long to allow wavevector matching at the boundary, it cannot couple to light propagating in air. Above $600 \mathrm{THz}$, the imaginary part of the in-plane component of the SPP wavevector becomes so large that the width of the dispersion curve crosses the light line. At these frequencies the SPP is not only damped because of Ohmic damping in the metal, but also because of radiation losses. As the dielectric constant in this region of frequencies is dominated by interband transitions of bound electrons instead of by the response of a free electron gas, the surface wave does not show the typical surface plasmon polariton characteristics. The frequency at which the denominator in Equation 2.1 becomes zero is called the surface plasmon resonance frequency. Theoretically, frequencies approaching this frequency will have very large wavevectors, leading to very small wavelengths. In principle this shortening of the wavelength enables very high confinement. For an interface between a pure Drude metal and air, this condition occurs at the frequency $\omega=\frac{\omega_{p}}{\sqrt{2}}$. In the case of a gold-air interface, this resonance lies in the UV and plays no role in the work described in this thesis. For a good understanding of the optical effects occurring in gold
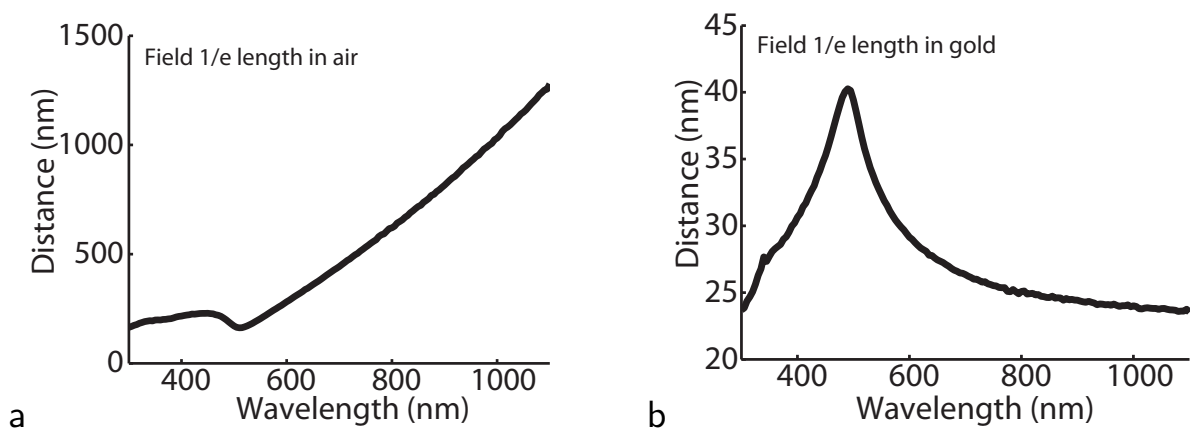

Figure 2.3: The SPP field extension in air (a) and gold (b). Values in the figures are calculated based on a measured value of the dielectric constant.

in the region from 400 to $1100 \mathrm{~nm}$, it is useful to be aware of a few other properties. In Figures 2.3 and 2.3, calculated values of some characteristic distances of a SPP on gold are plotted. Graphs 2.3a and 2.3b show the $1 /$ e extension of the field of surface plasmons into the air and into the gold respectively. Note that the extension in air is comparable to the wavelength while the extension in gold is limited to a few nanometer. Figure 2.4 shows 


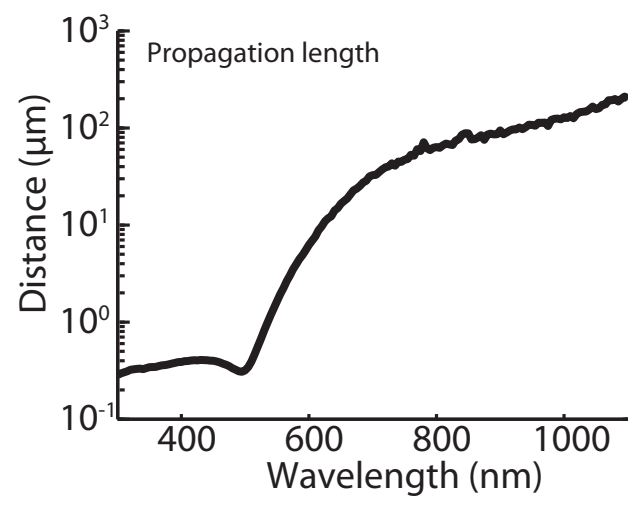

Figure 2.4: The SPP propagation length on a flat gold surface. Values in the figure are calculated based on a measured value of the dielectric constant.

the propagation distance of surface plasmons. In the region below $550 \mathrm{~nm}$ this distance is very short due to the high losses discussed in the previous paragraph.

\subsection{Localized surface plasmons}

In the interpretation of light interaction with nanoplasmonic structures, localized surface plasmons (LSPs) play a prominent role [14]. Localized surface plasmons are, as their name suggests, surface charge density oscillations that are localized in space. The clearest manifestation of LSPs are the resonances of small metallic particles [15] that are famous for their beautiful coloring of some glass stained windows. Assigning a resonance to a localized surface plasmon is useful, as the response expressed in the scattering or absorption of these structures has spectral features that often depend strongly on their geometry. However, one should keep in mind that these resonances are not of a high quality factor as in some other fields in photonics. This is due to the Ohmic damping and the intrinsic radiation losses of the LSP's. Whereas light cannot couple to surface plasmon polaritons, it can couple efficiently to LSP's due to the finite extent of these structures. A key property of localized plasmons is that they do not show dispersion, i.e., their response is insensitive to the magnitude of the 
wavevector of the field that excites them. This automatically limits them to sizes smaller than the wavelength. Other examples of systems in which localized plasmons play a role are bow tie antennas and sharp metal tips, both of which have been exploited in nonlinear optics $[7,16,17]$.

\subsection{Surface waves}

The past 10 years have shown a growing consciousness in the nano-plasmonics community that apart from surface plasmon polaritons other surface waves can also play a role in many of the observed phenomena. The nature of these surface waves is heavily debated in the community. This 'other' contribution is a wave that, for optical frequencies and on metals, is always present in combination with the surface plasmon polariton. At distances close to the source of both waves this wave dominates over the surface plasmon polariton. Its amplitude decreases however initially more quickly than the amplitude of the surface plasmon polariton. Throughout this thesis these waves will be referred to as "lossy surface waves".

During the past century, surface waves have been noticed several times by many different authors discussing for instance antenna theory. As early as 1909 Sommerfeld in his research on the propagation of radiowaves, found that surface waves were excited by dipoles above a surface [18]. His solutions and the existence of surface waves were however subject to debate for many decades $[19,20]$. In the 1980s Ford and Weber in their work on dipoles radiating above metal surfaces recognized that apart from a surface plasmon polariton other surface waves play a role as well and named these waves "lossy surface waves" [21]. Their interpretation is that these surface waves are associated with the near-field of the dipole. Lezec and Thio were the first to propose an additional type of surface wave in their explication of the extraordinary transmission effect and named them "composite diffracted evanescent waves" [22]. Their proposal lead to a lot of criticism [23], mainly because their underlying model was based on a scalar wave approximation. Generally, but especially in diffraction problems where metals are involved it is incorrect to use a scalar wave approximation and a full vectorial method should be used instead. Their claim of an additional surface wave was however well supported by experiments. Recent papers by Lalanne and co-workers propose yet another wave named "creeping wave" or "quasi-cylindrical wave" $[24,25]$. In these papers the scattering of waves 
at a slit is assumed to be well described by the radiation of a line dipole on the surface. From this treatment the nature of the waves in the mathematical sense became more clear, but still included a numerical solution of a rather complex integral. This was resolved by Gravel and co-workers [26] who presented an analytical description of these waves. Based on their findings the waves appear to have a character that is related to the surface plasmon and the authors interpret the waves as "transient surface plasmon polaritons".

It is important to realize that these waves are in full agreement with rigorous techniques to solve Maxwell's equations. All the numerical methods used in the community such as finite difference time domain (FDTD) calculations, boundary element method (BEM) or fourier model methods (FMM) fully incorporate these waves. For a more fundamental understanding of many effects in plasmonics and beyond, a physical interpretation of lossy surface waves is however highly desirable.

The physical interpretation of these waves is surely not as clear as it is for surface plasmon polaritons. The latter are homogeneous solutions or eigenmodes of a system consisting of two half spaces with a different dielectric index. In the formalisms used by Lalanne, Gravel and Ford and Weber, the Maxwell equations are solved for a source close to or on the interface between a half-space metal and a half-space dielectric. For this they use a wave expansion in the directions parallel to the surface. As a last step in finding the electromagnetic fields on the surface, they have to perform the Fourier-integral needed to go back from reciprocal to real space. Lalanne and Gravel use a contour integration in the complex plane of the integration variable $\left(k_{\|}\right)$to find the solution. In this integral SPPs emerge quite naturally from a pole in the reflection coefficient. The rest of the solution originates mathematically from a different type of singularity, a so called branch point [27]. This contribution to the field in real space contains the normal propagating light and the debated surface waves. Our current understanding is that these surface waves contain at least a contribution that arises from the large wavevectors a (for instance dipolar) source has close to its origin. These waves correspond to the response of the surface to the near-field of the source. This leads to a wave that at least initially decays faster than the surface plasmon polariton. Additionally there seems to be a contribution to the field on the surface that propagates much further. The interpretation of the waves and their role in many plasmonic systems will 
undoubtedly be subject of many discussions within the field for some time. However, the existence of lossy surface waves seems undeniable by now. As we will show in Chapter 4 lossy surface waves are excited in transition radiation microscopy as well. The naming of these waves in literature by different authors shows considerable dispersion. All the proposed names have their pro's and con's, in this thesis we will use the term lossy surface wave as was proposed by Ford and Weber. We will use the term surface wave, if we want to include all electromagnetic fields on the surface, i.e., lossy surface waves and surface plasmon polaritons.

\subsection{Extraordinary transmission}

The important role that subwavelength holes play in the nano-plasmonics field started with the discovery of the extraordinary transmission (EOT) effect by Ebbesen and co-workers [1]. The authors studied the transmission of light through a $200 \mathrm{~nm}$ thick silver film perforated by subwavelength holes arranged in a periodic array (see Figure 2.5). They observed sharp peaks in the transmission spectra at wavelengths many times larger than the size of the apertures. The overall transmission, normalized to the open surface of the structure, exceeded unity for specific wavelengths. This remarkable observation was in complete contradiction to the theoretical understanding of light transmission through subwavelength apertures at that time.

Soon after experiments showed the remarkable influence of hole shape on the transmission of these structures $[2,28,29]$. When one of the sides of rectangular holes was decreased, the peak in the transmission spectrum showed a large $(100 \mathrm{~nm})$ red shift. This was yet another surprise of extraordinary transmission, as one naively might expect the cutoff of a single hole to shift to lower wavelengths as the size of the hole is decreased. In this section on extraordinary transmission we will focus on rectangular holes. Firstly we will discuss the properties of these holes and secondly the transmission through hole arrays.

\subsubsection{Single subwavelength holes}

Already in the 1940s the diffraction of of light by subwavelength holes was studied theoretically, most notably in the works by Bethe and Bouwkamp $[30,31]$. They described the transmission of light through a subwavelength 


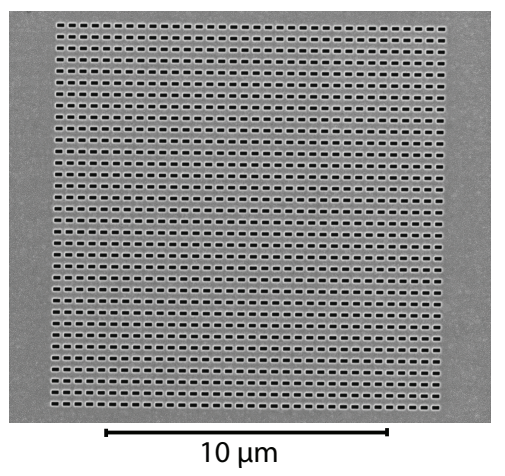

Figure 2.5: Scanning electron microscopy image of a subwavelength hole array in gold. The period of the array is $410 \mathrm{~nm}$ in both directions.

hole in an infinitely thin perfect conductor. Their works showed that the transmission through the holes scales as $(r / \lambda)^{4}$ with $r$ the radius of the hole and $\lambda$ the wavelength of the light. For holes of the size Ebbesen and co-workers experimented with, this led to a prediction for the transmission that was orders of magnitude smaller than what was observed. The remarkable experiment sparked a wealth of theoretical investigations that have severely changed the understanding of the transmission through an aperture. Notwithstanding the excellent work done by Bethe and Bouwkamp, their results were simply not applicable to the case of light transmission through a real metal layer of finite thickness.

The current view on transmission through a subwavelength hole in a film with a thickness larger than the penetration depth of light, is that the holes can be considered as waveguides. The modes of these waveguides can be described completely analytically for perfect conductors [32]. In this case, the lowest order mode of a square waveguide has a cutoff wavelength $\lambda=2 d$ where $\mathrm{d}$ is the width of of the waveguide. For wavelengths above this cutoff condition, transmission is exponentially damped. Due to the finite conductivity of a real metal, the penetration depth of the field in a real metal is larger than in a perfect conductor. This increases the cutoff wavelength of apertures in films made of for instance gold or silver, but it does not explain the high transmission observed for single apertures in the optical regime. 
A breakthrough came with the realization that the propagation through rectangular holes could be modeled accurately with two coupled surface plasmon polaritons on each side of the hole [33]. This type of mode was already known for some time as the mode of a metal-insulator-metal waveguide [34]. This mode has no cutoff in an infinite long slit. In a hole of finite width this is obviously not true and the mode has a cutoff. To get a better idea of this effect we show the influence of the hole shape on the cutoff frequency of the hole. A model for the cutoff wavelength as a function of the dimensions of the hole was proposed by Collin and co-workers [35] in which the cutoff wavelength is given by

$$
\lambda_{c}=2\left(w_{x}+2 \delta\right) \sqrt{\varepsilon_{a i r}\left(1+\frac{2 \delta}{w_{y}}\right)},
$$

with $\delta$ the skin depth of gold given by $\delta=\lambda /\left(4 \pi \sqrt{\varepsilon_{\text {gold }}}\right), w_{x}$ and $w_{y}$ the width and height of the hole. $\varepsilon_{\text {gold }}$ and $\varepsilon_{\text {air }}$ are the dielectric constant of the gold and the air respectively. This approximate model is derived by approximating the effective index of the waveguide mode and was shown to have good correspondence with finite element calculations. In Figure 2.6

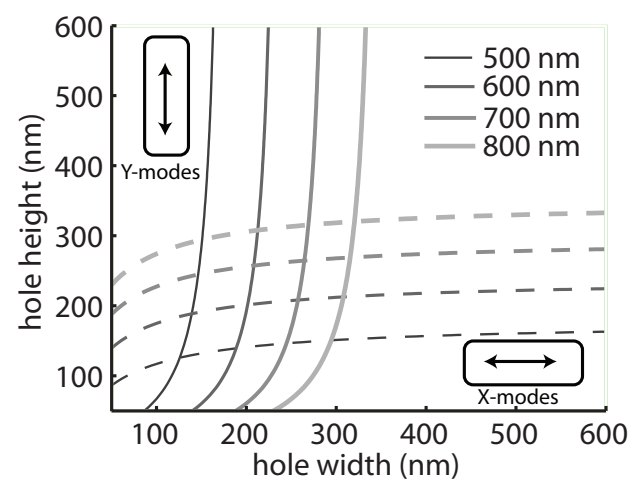

Figure 2.6: The cutoff for the lowest order mode for rectangular holes based on a model by Collin and co-workers [35]. The lines indicate the dimensions of holes with a cutoff wavelength of 500, 600, 700 and $800 \mathrm{~nm}$. The horizontal lines correspond to the $X$-mode, while the vertical lines correspond to the $Y$-mode.

the cutoff of the two orthogonal modes that can propagate in a rectangular 
hole are plotted as a function of the height for different wavelengths. The curves indicate the cutoff for different wavelengths. As we will be mainly interested in wavelengths between $400-900 \mathrm{~nm}$ in this thesis, we show the cutoff dimensions for wavelengths of 500, 600, 700 and $800 \mathrm{~nm}$. The Xmode oscillating along the width of the hole correspond to the horizontal lines for the cutoff dimensions of the hole (see Figure 2.6). Notice especially that the cut of for the X-mode of a hole of fixed height shifts to the red as the hole width decreases. This explains why a decreasing width of a rectangular hole leads to a red-shift of the observed transmission peak in extraordinary transmission $[2,28,29,36]$.

Additionally a resonance phenomenon was found to exist at the cutoff condition $[37,38]$. Here the propagation constant vanishes, thus the wavelength of the light propagating through the aperture becomes very large. The reflections at the two end facets of the hole lead to the formation of a zero- $\lambda$ Fabry-Perot resonance that further enhances the transmission. These resonances are often called localized resonances as they are localized in the hole and therefore show no dispersion. Different types of localized resonances were associated with the holes by several authors, where most consider resonances in the holes $[39,37,38]$ and some a localized surface plasmon on the edge of the hole [2].

The ability to launch SPPs with the apertures plays a vital role in the explanation of the extraordinary transmission phenomenon, therefore this has been separately investigated by several authors, both experimentally $[40,41]$ and theoretically $[42,43,44]$. The common result of these publication is that a high efficiency of surface plasmon polariton excitation is possible with a subwavelength hole [41]. Additionally it was shown that the emission of a circular hole has a dipolar origin due to the opposite charge accumulation at both sides of the hole [42]. A thorough investigation of the surface plasmon polariton launching properties of single holes was done by Baudrion and co-workers [41]. They measured and calculated the coupling efficiency of incident light to SPPs on a gold-air interface with square holes of different size. They found that for a wavelength of $800 \mathrm{~nm}$, the coupling to surface plasmons peaks at holes with a width of $200 \mathrm{~nm}$, where they found a normalized efficiency of $25 \%$. For smaller holes they observe a steep decrease of the coupling efficiency to $5 \%$ at holes with a width of 100 nm. For large holes the decrease is less steep and for holes with a width of $500 \mathrm{~nm}$ the measured efficiency is $15 \%$. They associate the peak in 
efficiency with the cutoff of a hole. Beyond cutoff the efficiency is very low, at cutoff it is maximum and above cutoff light can couple to the hole and the scattering efficiency decreases slowly with wavelength. The coupling to the far-field of the surface wave occurs thus to have a relation to the modes of the hole.

\subsubsection{Arrays of subwavelength holes}

The development in understanding of the transmission through single subwavelength holes went parallel with the developments in the extraordinary transmission (EOT) phenomenon, in which a periodic array of holes plays a role $[45,46,47,48]$. Currently the view that is shared amongst most researchers is that the EOT phenomenon involves surface waves propagating on both interfaces, propagating or tunneling modes through the holes and free space light modes on both sides of the sample. The coupling between the surface waves on both interfaces via the holes and to the continuum of states available in the media on both sides of the structure gives rise to transmission spectra with a typical Fano shape [49, 50]. The exact outcome of the coupling between the different modes is critically dependent on the conditions of which the periodicity is the most prominent. The surface waves on both sides of the hole in the original paper were surface plasmon polaritons but this is not necessary. It is widely recognized that surface plasmon polaritons are responsible for EOT in most experiments in the optical regime $[51,52]$. Yet, in principle any other surface wave can lead to the same phenomenon $[53,25]$.

The dynamics of the resonances present in the structure can be studied by characterizing the temporal shape of the pulses propagating through hole arrays. Several studies have been performed both experimentally and theoretically, that have confirmed the resonance properties that have been ascribed to EOT $[54,55,56]$. The delay observed in these measurements is in the range -40 to $10 \mathrm{fs}$. Noteworthy to the studies in this thesis, the delay introduced by the propagation through the hole was found to be negligible with respect to the total delay [54].

The high fields present in hole arrays due to the plasmonic modes on the surface and in the holes, lead to nonlinear effects. Bistability was observed in hole arrays coated with a nonlinear polymer [57]. Second harmonic generation (SHG) was observed by illuminating the holes with high intensity femtosecond laser pulses [58]. Nieuwstadt and coworkers investigated the 
role of hole shape on the second harmonic generation efficiency. Varying the aspect ratio of rectangular holes in an array, a specific 'hot' hole shape was found to exist at which the SHG efficiency increased by an order of magnitude. It was suggested that this was related with a lower group velocity for that specific hole shape. One curious detail in these experiments is that in the idealized case the samples under investigation have inversion symmetry. SHG is not expected in bulk materials with this symmetry [59]. Note that at the surface of a inversion symmetric structure however, SHG is allowed [60] due to amongst other things quadrupole contributions. It is unknown whether the SHG observed in these experiments is related to small roughnesses of the gold or to a quadrupole contribution to the SHG. 


\section{Chapter 3}

\section{Group delay through subwavelength hole arrays}

The role of hole shape in the dynamic properties of light interacting with arrays of rectangular subwavelength holes is investigated and related with nonlinear effects occurring in the structures. It is shown that the group delay of a femtosecond pulse propagating through a hole array depends on the shape of the hole. The observed delay has a maximum near the cutoff frequency of the holes. Additionally it is shown that the amount of nonlinear second harmonic generated in the sample increases with group delay.

\subsection{Introduction}

After the discovery of the extraordinary transmission effect [1], many researchers started to investigate the physics of subwavelength hole arrays. Soon, experiments showed the remarkable large influence of hole shape [28]. When one of the sides of a rectangular hole was shortened, the transmission showed a large red shift. This was completely contrary to expectations at that time, as one would naively expect the cutoff of a single hole to shift to lower wavelengths as the size of the hole is decreased. Theoretical work in the years thereafter, showed that the transmission through a rectangular hole is significantly influenced by surface plasmon polaritons in the hole $[37,38,33]$. Their role in the transmission properties of rectangular holes, leads to a counter intuitive red shift of the cutoff wavelength when the height of a hole is decreased (see Section 2.6.1). Additionally a special 
condition was shown to exist at cutoff, where a Fabry-Perot like resonance is expected. Although the dynamics of light propagation through subwavelength holes has been thoroughly studied (see Section 2.6.2), the role of hole shape and the dynamic properties of the resonances of the rectangular hole, has however been experimentally untouched until now.

Since surface plasmon polaritons are bound to the surface, high electromagnetic fields are present directly on the surface of the metal. This has led to an interest in hole arrays for enhancing nonlinear effects [58]. In the work by van Nieuwstadt and co-workers [61] the role of hole shape on the second harmonic generation (SHG) efficiency of hole arrays was investigated. It was found that there exists a 'hot' hole shape for which the SHG efficiency is an order of magnitude higher then for other hole shapes. It was suggested that this striking effect is related to the group velocity in these structures. In a Fabry-Perot type resonator, the intensity in the medium rises linearly with the delay. Since the amount of second harmonic generated in a medium scales with the square of the intensity, the amount of second harmonic generated is expected to rise quadratic with the delay through the medium. A direct link between the nonlinear effect and the group delay has however not been shown in these structures yet.

In this chapter we investigate the role of hole shape on the dynamic properties of subwavelength hole arrays and the nonlinear second harmonic generated. Using an interferometric technique we will measure the group delay of femtosecond pulses through arrays of subwavelength holes. In a separate experiment we will investigate the role of hole shape on the SHG effect.

\subsection{Subwavelength hole array structures}

The samples under investigation are arrays of $34 \times 34$ holes milled with a focused ion beam in a $200 \mathrm{~nm}$ thick gold layer on a glass substrate (Figure 3.1). The period of the square lattice array is fixed at $410 \mathrm{~nm}$. The dimensions of the holes varies from $205 \times 141$ to $328 \times 88 \mathrm{~nm}$, keeping the open surface of the arrays constant at $33 \mu \mathrm{m}^{2}$. Thus, the range of aspect ratios of the holes runs from 1.46 to 3.73 and is chosen such that the range crosses the condition for cutoff for wavelengths around $800 \mathrm{~nm}$, as can be checked in Figure 2.6. The total The accuracy with which the dimensions of these holes can be determined is limited, due to the difficulty in defining 
the position of the edge in scanning electron microscopy or atomic force microscopy data. For this reason the dimensions used in the presentation of the results are the original design parameters. These deviate from the real dimensions mostly by a small $(10-20 \mathrm{~nm})$ systematic error that arises from he spot size and magnification factor used during focused ion beam milling. For calibration purposes every hole array is accompanied by a large reference hole with the same outer dimensions as the whole array, i.e., 14 x $14 \mu \mathrm{m}$. The structures and reference holes are placed $70 \mu \mathrm{m}$ apart.

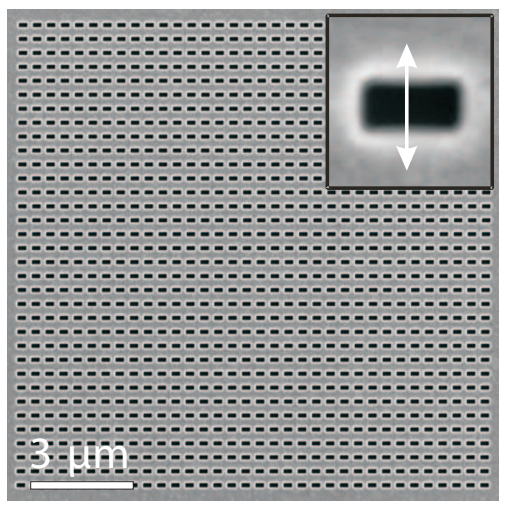

Figure 3.1: Scanning electron microscopy image of a hole array with sides: $W=246 \mathrm{~nm}$ and $H=117 \mathrm{~nm}$, the aspect ratio $W / H$ is 2.1. The inset shows a zoom in of an individual hole. The polarization is oriented along the short axis of the holes as indicated by the white arrow.

\subsection{Experimental technique}

To determine the group delay we use an interferometric technique. The sample is mounted in one of the branches of a Mach-Zehnder interferometer (see Figure 3.2). A femtosecond laser pulse is sent into the interferometer and an interferometric signal is detected. Changing the path length of the reference branch, allows us to measure the interferogram of the femtosecond pulse (Figure 3.3). The position of the maximum in this interferogram changes as the delay in the sample branch changes. The relative pulse delay can be determined by comparing the position of the interferogram 
when the pulse propagates through a sample with the position of the interferogram when the pulse propagates through a reference structure. To obtain amplitude and phase of the interference signal simultaneously we use a heterodyning lock-in technique [62] in which the light in the reference branch is shifted $9 \mathrm{MHz}$ using two acousto-optic modulators. From the detected signal we construct the complex interferogram. The delay line used to measure the interferogram is a pair of mirrors mounted on a linear motor (Newport XMS50) with an encoder (Heidenhain LIF 481). The positioning of this stage is specified to have a minimum incremental motion of $10 \mathrm{~nm}$ and a resolution that is tenfold smaller than this. To compensate for the dispersion introduced by the two acousto-optic modulators, a compensating crystal is mounted in the other branch. The light source used is a Ti:sapphire mode-locked laser (Spectra Physics Tsunami) that generates $100 \mathrm{fs}$ pulses at a $80 \mathrm{MHz}$ repetition rate, tunable in the range 760-830 $\mathrm{nm}$. The polarization of the light in the experiment is rotated with a $\lambda / 2$ waveplate to orient it parallel to the short axis of the holes. The light is focused and collected using two lenses with a numerical aperture of 0.4. The focus is determined to be smaller than $2 \mu \mathrm{m}$ by imaging the sample and the focus on a CCD camera. The sample is mounted on a X-Y piezo arm that moves the sample perpendicular to the impinging laser beam. This allows us to rapidly alternate the measurements between the hole array and the reference hole. A single interferogram is acquired within half a second. Alternately, the hole array and the reference hole are measured and this cycle is repeated 100 times. This method allows us to monitor and possibly remove a drift in the path lengths of the setup. To accurately determine the delays, the obtained interferograms are further processed by filtering in the frequency domain. The signal is Fourier transformed and low frequency noise sources of electronic origin are removed. To determine the delay, a Gaussian is fitted to the amplitude of each filtered interferogram. Of each structure more than 100 interferograms are processes as described. From the obtained delays an average delay and a spread in delay is determined.

The second harmonic generated on a sample is measured in a separate experiment. The light is focused on the sample with a NA of 0.17 and collected with a higher NA. The sample is tilted under a small angle of 2.5 degree to prevent back reflections from reaching the mode-locked pulsed laser. We verified in a separate experiment that the results depend only very weakly on this angle. The transmitted fundamental wavelength is 
attenuated by a combination of colored glass and interferometric filters. The generated light is measured with a spectrometer (Acton SpectraPro 2300i) equipped with a cooled CCD camera (Princeton Instruments Spec10B/XTE). Spectra are typically collected in 200 seconds.

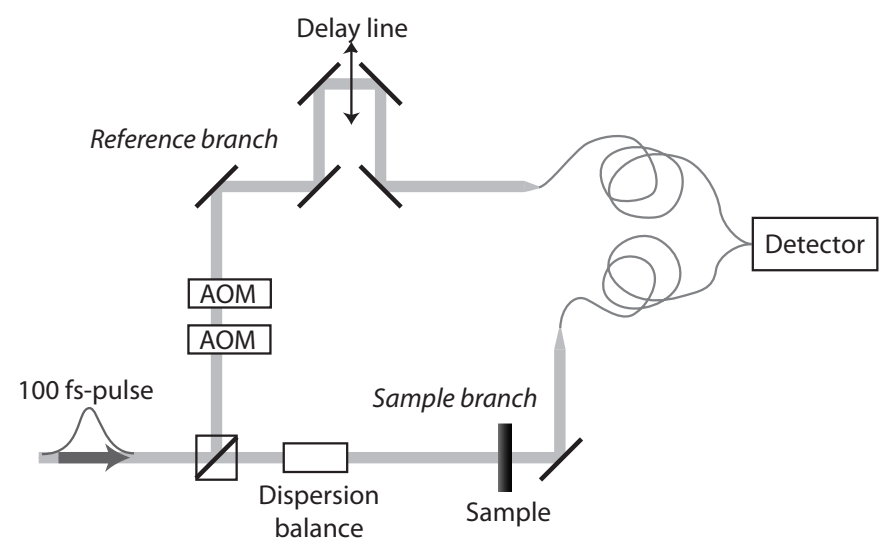

Figure 3.2: The interferometric setup used to determine the group delay. Femtosecond pulses from a Ti:sapphire laser propagate through a MachZehnder interferometer. In the reference branch two acousto-optic modulators (AOM) are placed to shift the frequency of the light by $9 \mathrm{MHz}$. A delay line is used to measure the interferogram. In the signal branch a sample is placed plus a dispersion compensating crystal to balance the dispersion introduced by the two acousto-optic modulators in the reference branch. Both signals are coupled into a 2 by 1 fiber coupler.

\subsection{Experimental results}

In Figure 3.4 the group delay is plotted as a function of aspect ratio for 4 different wavelengths. The origin of the time axis is given by the delay observed for pulses propagating through the reference holes. For all wavelengths and aspect ratios a positive delay is found with respect to a transmission through a reference structure. The delays observed range from 0.5 to 5.5 fs. Figure $3.4 \mathrm{a}$ and $3.4 \mathrm{~b}$ for 760 and $780 \mathrm{~nm}$ illumination both show a decreasing delay as the aspect ratio increases. The delay for pulses of $810 \mathrm{~nm}$ (Figure 3.4c) shows a faint peak near aspect ratio 2. Figure $3.4 \mathrm{~d}$ 


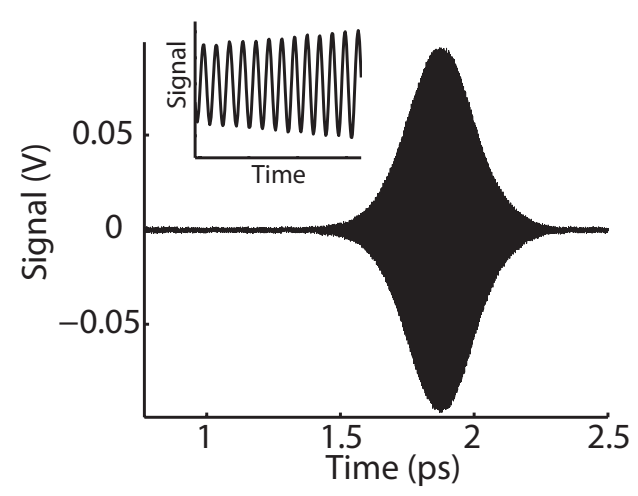

Figure 3.3: Typical interferogram, inset shows a zoom in of the interferogram.

shows the results for pulses with a center wavelength of $830 \mathrm{~nm}$. Here a clear peak can be observed at aspect ratio 2 .

We define the maximum enhancement of the group delay as the maximal ratio between the observed transit times of the pulse through the structures:

$$
f=\frac{\Delta T_{\max }}{\Delta T_{\min }}=\frac{\tau_{\max }+\tau_{\text {offset }}}{\tau_{\min }+\tau_{\text {offset }}}
$$

The minimum group delay $\tau_{\min }$ we find in our measurements is $1 \mathrm{fs}$. The maximum delay $\tau_{\max }$ is $4.5 \mathrm{fs}$. Since these delays are relative to a reference pulse propagating through air, for the real transit time of the pulse we need to add $\tau_{\text {offset }}=0.66 \mathrm{fs}$, the transit time of a pulse in air over a distance of 200 $\mathrm{nm}$ corresponding to the metal thickness. The enhancement of the pulse delay is therefore a factor 3 .

\subsection{Finite difference time domain calculations}

We used finite difference time domain (FDTD) calculations to complement our experimental findings of the group delay. With a commercial finite difference time domain package (CST Microwave Studio [63]). The transmission of an ultra short femtosecond pulse through an infinite array of subwavelength holes was calculated for various aspect ratios of the holes. The dielectric constant of gold in this calculation was described with a 


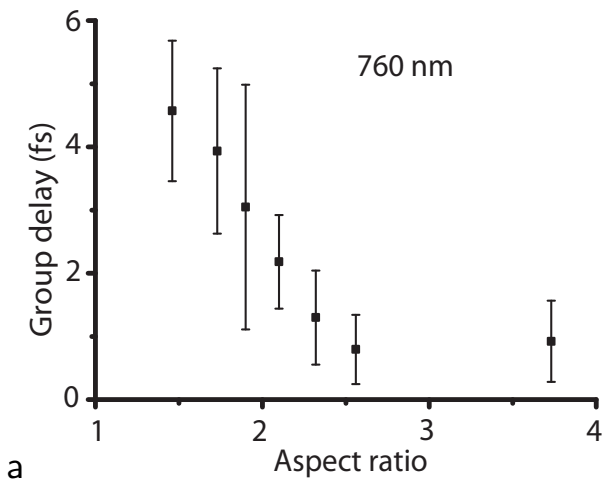

a

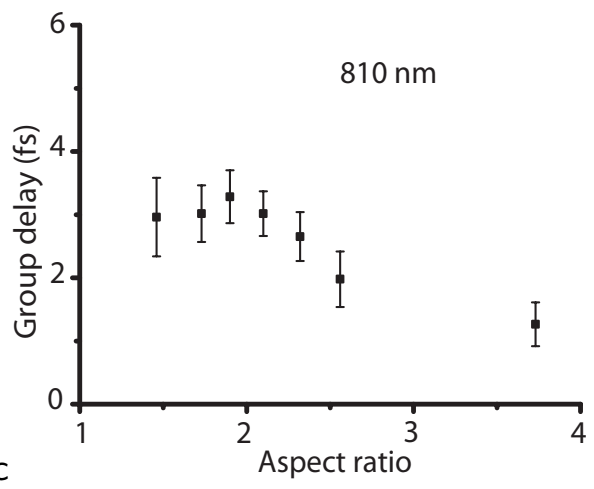

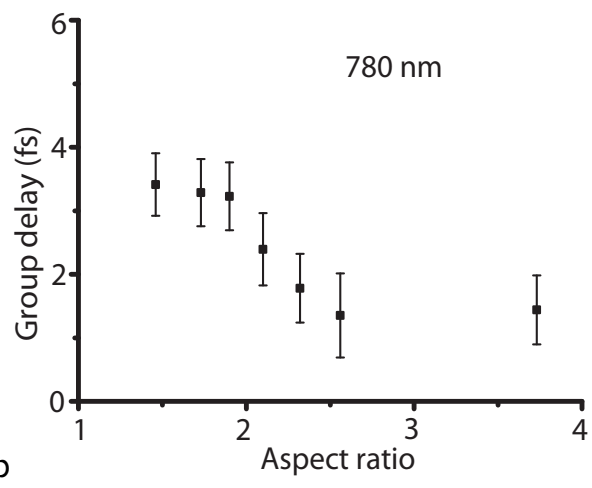

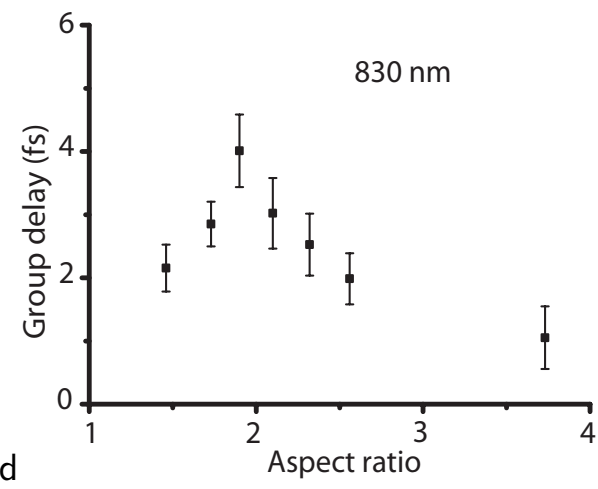

Figure 3.4: The group delay measured for hole arrays with different aspect ratio holes for a laser wavelength centered around a) $760 \mathrm{~nm}$, b) $780 \mathrm{~nm}$, c) $810 \mathrm{~nm}$, d) $830 \mathrm{~nm}$. The error bars are the standard deviation over 100 measurements. The zero delay corresponds to a measurement on a large reference hole. 
Drude model $\left(\varepsilon_{\infty}=6.3718, \omega_{p}=1.249410^{16} \mathrm{rad} / \mathrm{s}, \Gamma=0.014210^{16} \mathrm{~Hz}\right)$. The calculated field before and after the structure, respectively $E_{i n}(t)$ and $E_{\text {out }}(t)$, are Fourier transformed and used to determine the complex transfer function of the structures via $T(\omega)=\mathfrak{F}\left[E_{\text {out }}\right] / \mathfrak{F}\left[E_{\text {out }}\right]$. From which the group delay can be determined as $\tau_{g}=\mathrm{d} \arg [T(\omega)] / \mathrm{d} \omega$. The result of these calculations is shown in Figure 3.5. There is a convincing match between the measurements (see Figure 3.4) and the FDTD results. In both experiment and calculation the delay varies roughly between 0 and 6 femtoseconds. The trend of the group delay as a function of aspect ratio in both cases shows a maximum, that shifts towards higher aspect ratio for longer wavelengths. The slight deviation of the calculated values from the observed values is attributed to the small uncertainty in the experimental determination of the geometry of the holes.

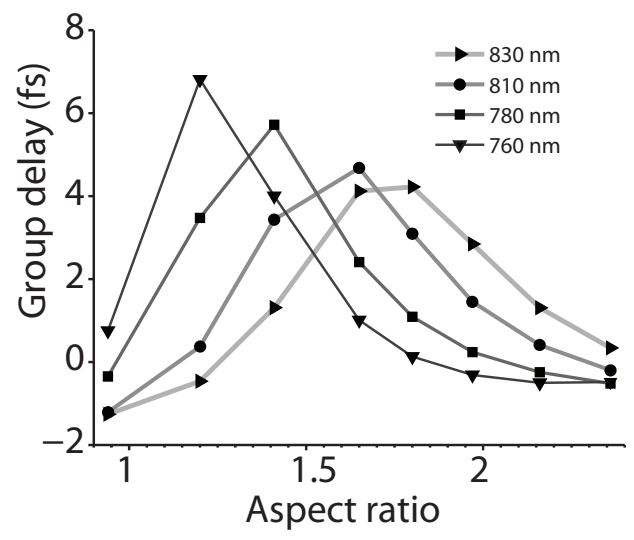

Figure 3.5: The group delay as calculated with FDTD technique as a function of aspect ratio for 4 different wavelengths, $760 \mathrm{~nm}$ (grey), $780 \mathrm{~nm}$ (red), $810 \mathrm{~nm}$ (green) and $830 \mathrm{~nm}$ (blue).

\subsection{Second harmonic generation}

In Figure 3.6 the results of the SHG experiment are plotted. Figure 3.6a shows the second harmonic generated intensity and the transmitted fundamental intensity as a function of the incoming fundamental power. As 

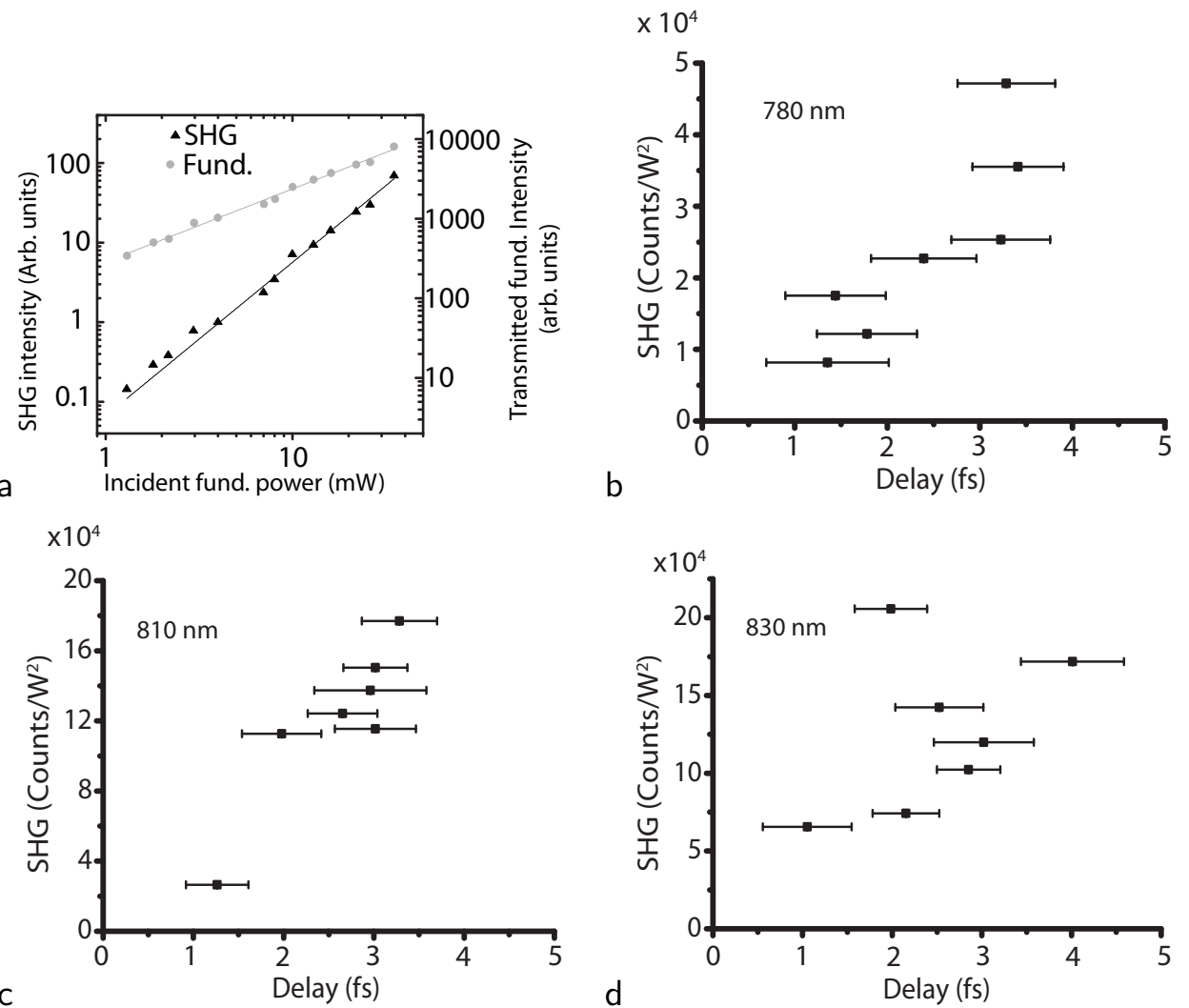

Figure 3.6: The SHG signal versus the incident power shows a quadratic dependence a). The $S H G$ generated versus the measured group delay for three different wavelengths , b) $780 \mathrm{~nm}$, c) $810 \mathrm{~nm}$, d) $830 \mathrm{~nm}$. All three graphs show an increase of the SHG signal as the group delay increases. 
expected the SHG intensity shows a quadratic dependence on the fundamental input power. Figures 3.6a, b and c show the SHG signal normalized to the incoming fundamental power squared as a function of group delay for 3 different wavelengths. In all three graphs the SHG signal shows a strong increase with group delay. This is in good agreement with our hypothesis that the group delay leads to an increase in nonlinear effects. Unfortunately, due to the small delays observed it is not possible to verify the exact relation ship between group delay and the nonlinear effect from the measured data.

\subsection{The role of attenuation}

When determining the group delay from either measured or calculated data one has to be careful in case attenuation plays a role. The reason is that a combination of group velocity dispersion and attenuation can cause a delay of the pulse envelope in addition to the delay caused by the group velocity itself. As the group delay is expected to play a role in the SHG efficiency of the hole array, it is important to find out how large the effect of attenuation is on the observed delay. To do this we emulate the performed experiment with the help of the results obtained in the FDTD calculation. We determine the delay of a 100 femtosecond Fourier-limited pulse $E_{i n}(\omega)$, using the transfer function $T(\omega)$ and the normalized transfer function $N(\omega)=T(\omega) /|T(\omega)|$. To determine the electric field of the transmitted pulse in the time domain we calculate $E_{\text {out }}(t)=\mathfrak{F}^{-1}\left[E_{\text {in }}(\omega) T(\omega)\right]$. From the calculated value of $E_{\text {out }}(t)$, the group delay is determined. This procedure is repeated for the normalized transfer function. The difference in delays calculated for the normalized and not normalized transfer function is always smaller than 150 attoseconds, well within the experimental error. More information on the role of attenuation, also in case of not Fourier-limited pulses and with the interferometric technique used, is given in Appendix A.

\subsection{Conclusion}

We have investigated the influence of hole shape on the group delay of femtosecond pulses propagating through hole arrays. We observed a maximum delay that shifts to larger wavelengths for holes with larger aspect ratio. 
This is in agreement with theoretical work that predicts the presence of a resonance at the cutoff frequency of subwavelength holes [64]. By tuning the aspect ratio of the holes, the cutoff frequency of the array is shifted. This shifts the associated resonance through the measurement window. Additionally we observed that as the group delay of the pulse increases the amount of second harmonic generated rises. We interpret the result as a strong indication that the second harmonic is generated in the localized resonances present in holes. 
Chapter 3 


\section{Chapter 4}

\section{Transition radiation}

The radiation generated by an electron moving through the interface between two media with a different dielectric constant is derived. The aim is to investigate how this transition radiation can be used as a tool to study nanoplasmonic structures.

When a charged particle moves through a medium with a velocity higher than the velocity of light in the medium it will radiate light. This radiation is called Cherenkov radiation and for the discovery and interpretation of the effect Cherenkov, Tamm and Frank received the 1958 Nobel prize. A different effect occurs when a charged particle passes through the interface between two materials with different dielectric constants. Then, irrespective of the speed of the particle, radiation is generated. This light is called transition radiation and was first theoretically described by Ginzburg and Frank in 1944 [65, 5].

In this chapter the generation mechanism of transition radiation is explained. The analysis yields insight into the relevant spatial length scales on which transition radiation is generated. The result is important for the understanding of the experimental work presented later in this thesis. In the first three sections the field of a moving point charge and the generated transition radiation field are derived and interpreted. In the fourth section a comparison is made between transition radiation and the radiation from a dipole source. The chapter ends with a discussion of the results in the perspective of using the transition radiation as an experimental technique in nano-optics. 


\subsection{The field of a moving point charge}

To derive the field of a charged particle moving through the interface between two media, we first derive the field in each medium separately. We will consider a point charge $q$ moving with a non-relativistic constant velocity $\mathbf{v}$ along the $z$-axis (see Figure 4.3). It is assumed that $\mu=1$ everywhere in space, i.e., the materials we consider are non-magnetic. The source terms

$\varepsilon$
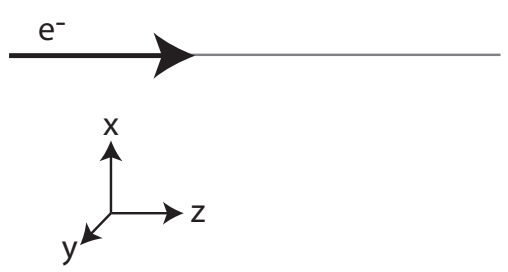

Figure 4.1: A charge moving along the z-direction in a homogeneous medium with dielectric constant $\varepsilon$.

in the Maxwell equations are

$$
\begin{gathered}
\rho=q \delta(z-v t) \delta\left(x_{0}\right) \delta\left(y_{0}\right) \text { and } \\
\mathbf{J}=q \mathbf{v} \delta(z-v t) \delta\left(x_{0}\right) \delta\left(y_{0}\right) .
\end{gathered}
$$

Where $\rho$ and $\mathbf{J}$ are the charge and current distribution. $v$ is the absolute value of the velocity vector $\mathbf{v}$. To calculate the field of a moving point charge we will use the vector potential $\mathbf{A}$ defined as

$$
\mathbf{H}=\nabla \times \mathbf{A} .
$$

where $\mathbf{H}$ is the magnetic field. Combined with Maxwell equation for the curl of $\mathbf{E}$ it is possible to write the electric field as

$$
\mathbf{E}=-\mu_{0} \frac{\partial \mathbf{A}}{\partial t}-\nabla \phi
$$

In which $\phi$ is a scalar potential. It will turn out to be convenient to use the Lorenz gauge:

$$
\nabla \cdot \mathbf{A}+\varepsilon_{0} \varepsilon \frac{\partial \phi}{\partial t}=0
$$


Substituting 4.3 and 4.4 in the Maxwell equation for the curl of $\mathbf{H}$ and using the Lorenz gauge yields one wave equation for the scalar potential and one for the vector potential,

$$
\begin{gathered}
\nabla^{2} \mathbf{A}-\frac{\varepsilon}{c^{2}} \frac{\partial^{2} \mathbf{A}}{\partial t^{2}}=-q \mathbf{v} \delta(z-v t) \delta\left(x_{0}\right) \delta\left(y_{0}\right) \text { and } \\
\nabla^{2} \phi-\frac{\varepsilon}{c^{2}} \frac{\partial^{2} \phi}{\partial t^{2}}=-\frac{q}{\varepsilon_{0} \varepsilon} \delta(z-v t) \delta\left(x_{0}\right) \delta\left(y_{0}\right) .
\end{gathered}
$$

It is useful to perform a Fourier expansion of these expressions in time and in the two spatial directions $\mathbf{x}$ and $\mathbf{y}$ perpendicular to the charge velocity. Performing these expansions leads to

$$
\begin{aligned}
k_{x}^{2} \mathbf{A}_{k, \omega}+k_{y}^{2} \mathbf{A}_{k, \omega}-\frac{\partial^{2} \mathbf{A}_{k, \omega}}{\partial z^{2}}-\frac{\varepsilon}{c^{2}} \omega^{2} \mathbf{A}_{k, \omega} & =\frac{\mathbf{v}}{v} e^{i\left(\frac{\omega}{v} z\right)} \text { and } \\
k_{x}^{2} \phi_{k, \omega}+k_{y}^{2} \phi_{k, \omega}-\frac{\partial^{2} \phi_{k, \omega}}{\partial z^{2}}-\frac{\varepsilon}{c^{2}} \omega^{2} \phi_{k, \omega} & =\frac{q}{\varepsilon_{0} \varepsilon v} e^{i\left(\frac{\omega}{v} z\right)}
\end{aligned}
$$

where we have chosen (without loss of generality) $x_{0}=0$ and $y_{0}=0$. The somewhat unusual exponent on the right hand side is due to the expansion of $\delta(z-v t)$. The subscripts $\omega$ and $k$ indicate that both potentials are now a function of these two variables. For simplicity of notation these subscripts will be omitted in the following derivation. The forced solutions to the two inhomogeneous wave equations are

$$
\begin{gathered}
\mathbf{A}=\frac{q}{c} \frac{\mathbf{v}}{v}\left(k_{x}^{2}+k_{y}^{2}+\frac{\omega^{2}}{v^{2}}-\frac{\varepsilon}{c^{2}} \omega^{2}\right)^{-1} e^{i\left(\frac{\omega}{v} z\right)} \text { and } \\
\phi=\frac{q}{\varepsilon_{0} \varepsilon v}\left(k_{x}^{2}+k_{y}^{2}+\frac{\omega^{2}}{v^{2}}-\frac{\varepsilon}{c^{2}} \omega^{2}\right)^{-1} e^{i\left(\frac{\omega}{v} z\right)} .
\end{gathered}
$$

Substituting both solutions in Equation 4.4 yields the electric field of a point charge $q$ moving with constant velocity in a homogeneous medium

$$
\mathbf{E}_{q}=\frac{q i}{\varepsilon_{0} \varepsilon v} \frac{\omega \mathbf{v}\left(\frac{\varepsilon}{c^{2}}-\frac{1}{v^{2}}\right)-\mathbf{k}_{r}}{k_{r}^{2}+\omega^{2}\left(\frac{1}{v^{2}}-\frac{\varepsilon}{c^{2}}\right)} e^{i\left(\frac{\omega}{v} z\right)},
$$

where the in-plane wavevector is given by $\mathbf{k}_{r}=\mathbf{k}_{x}+\mathbf{k}_{y}$. This equation can be separated in components perpendicular $\left(\mathbf{E}_{z}\right)$ and parallel $\left(\mathbf{E}_{r}\right)$ to the surface:

$$
E_{z, q}=\frac{-q i}{\varepsilon_{0} \varepsilon} \frac{\omega\left(\frac{1}{v^{2}}-\frac{\varepsilon}{c^{2}}\right)}{k_{r}^{2}+\omega^{2}\left(\frac{1}{v^{2}}-\frac{\varepsilon}{c^{2}}\right)} e^{i\left(\frac{\omega}{v} z\right)} \text { and }
$$




$$
E_{r, q}=\frac{-q i}{\varepsilon_{0} \varepsilon v} \frac{k_{r}}{k_{r}^{2}+\omega^{2}\left(\frac{1}{v^{2}}-\frac{\varepsilon}{c^{2}}\right)} e^{i\left(\frac{\omega}{v} z\right)}
$$

These results will be used later to derive the transition radiation. It is however interesting to take a closer look at these equations. In Figure 4.2 the amplitude of $E_{z}$ is plotted as a function of $\omega$ and $k_{r}$ in a dispersion diagram for two values of the charge velocity. For the right graph there is a large amplitude above the light line visible that corresponds to light radiated in one specific direction. This contribution arises from the pole in Equation 4.13 and 4.14

$$
k_{r}^{2}+\omega^{2}\left(\frac{1}{v^{2}}-\frac{\varepsilon}{c^{2}}\right)=0
$$

We can derive the dispersion of this contribution by following this pole in the $\omega-k_{r}$ plane. This yields

$$
k_{r, p o l e}=\frac{\omega}{c} \sqrt{\varepsilon-\frac{c^{2}}{v^{2}}}
$$

If we assume $\varepsilon$ is real, the parallel wave vector $k_{r}$ will be imaginary for velocities of the charged particle below the phase velocity of light in the medium $\left(v<\frac{c}{\sqrt{\varepsilon}}\right)$. For $v>\frac{c}{\sqrt{\varepsilon}}$ the parallel wavevector becomes real i.e. the charge starts to radiate. This condition is exactly the condition for Cherenkov radiation.

The generation of Cherenkov radiation is also visible in the real space expressions for the fields of a moving charge. These real space expressions can be found by performing the inverse Fourier expansion in the $\mathrm{x}$ - and y-direction. This unfortunately has no closed expression in Cartesian coordinates, therefore we derive the result in cylindrical coordinates, using a Fourier-Bessel Transform. For the $z$-oriented field this leads to

$$
E_{z, q}=\frac{-q i}{\varepsilon_{0} \varepsilon} \mathrm{e}^{i \omega \frac{z}{v}} K_{0}\left(r \omega \sqrt{\frac{1}{v^{2}}-\frac{\varepsilon}{c^{2}}}\right)
$$

with $K_{0}$ the modified Bessel function of the second kind and $r$ the radial position. For the field in the radial direction the Fourier Bessel transform 

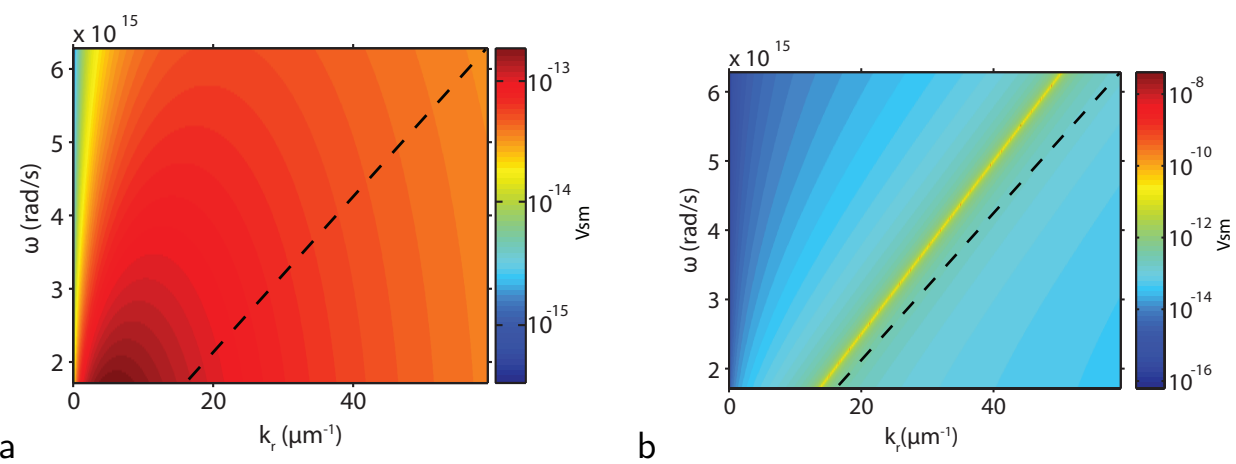

Figure 4.2: The amplitude of the electric field $E_{z}$ as a function of the parallel momentum $k_{r}$ and the frequency $\omega$, the figures can thus be considered dispersion diagrams. In both graphs the dielectric constant of the medium is $\varepsilon=8$. Notice that the color scale is logarithmic. a) Electric field for an electron velocity of $\frac{1}{3} c$. b) Electric field amplitude for an electron velocity of $\frac{2}{3}$. When the velocity is above the phase velocity of light in the medium Cherenkov radiation is generated. The black dashed line is the light line in the medium. 
leads to

$$
\begin{aligned}
E_{r, q}=\frac{-q i}{v \varepsilon_{0} \varepsilon} \mathrm{e}^{i \omega \frac{z}{v}}( & -\frac{1}{2} \pi \omega \sqrt{\frac{1}{v^{2}}-\frac{\varepsilon}{c^{2}}} I_{0}\left(r \omega \sqrt{\frac{1}{v^{2}}-\frac{\varepsilon}{c^{2}}}\right) \\
& \left.+r^{-1}{ }_{1} F_{2}\left(1 ; \frac{1}{2}, \frac{1}{2} ; \frac{1}{4} r^{2} \omega^{2}\left(\frac{1}{v^{2}}-\frac{\varepsilon}{c^{2}}\right)\right)\right),
\end{aligned}
$$

with $I_{0}$ the modified bessel function of the first kind and ${ }_{1} F_{2}$ a hypergeometric function. For a real valued argument, both $I_{0}$ and $K_{0}$ are monotonically decreasing functions and have no oscillating terms. This is in agreement with the fact that the field of an electron moving with constant speed is not radiative. The hypergeometric function is also decreasing monotonically as a function of radius. The modified Bessel function of the first kind $I_{0}$ contains the term $\sqrt{\frac{1}{v^{2}}-\frac{\varepsilon}{c^{2}}}$ in its argument. When the velocity becomes higher than the phase velocity in the medium i.e. $v>\frac{c}{n}$ this term becomes imaginary. An imaginary component in the argument of the modified Bessel functions of the first kind will lead to an oscillatory behavior, i.e. the field will become radiative. For the conditions we are interested in in this thesis the Cherenkov radiation will turn out to be a minor effect. For gold as a medium at wavelengths above $500 \mathrm{~nm}$ and an electron velocity of $\frac{c}{3}$ the imaginary part of the term $\sqrt{\frac{1}{v^{2}}-\frac{\varepsilon}{c^{2}}}$ is less than 5 percent of the real part of this term. In vacuum this term is obviously completely real. Therefore, within the experiments described in this thesis very little Cherenkov radiation is expected to be generated. However, the derivation of transition radiation in the next section will not make any assumptions that will exclude Cherenkov radiation.

\subsection{The field of the transition radiation}

In the previous section we have seen that the field of the electron depends on the dielectric constant $\varepsilon$. Transition radiation occurs because of the mismatch between the field of the electron above and below the interface separating two media with different dielectric constant. In a mathematical sense, the expression for transition radiation is derived by imposing that the field of the electron in the two media plus the field of the transition radiation should fulfill the usual boundary conditions at the interface. Please note 
that as a consequence, it is the interface that acts as the source for the transition radiation. The field of the transition radiation in both media is described by

$$
\begin{gathered}
E_{t r, z}=a_{1,2} \mathrm{e}^{ \pm \mathrm{i} z \sqrt{\frac{\varepsilon_{1,2} \omega^{2}}{c^{2}}-k_{r}^{2}}} \text { and } \\
\mathbf{E}_{t r, r}=\mp a_{1,2} \frac{\mathbf{k}_{\mathbf{r}}}{k_{r}^{2}} \sqrt{\frac{\varepsilon_{1,2} \omega^{2}}{c^{2}}-k_{r}^{2}} \mathrm{e}^{ \pm \mathrm{i} z \sqrt{\frac{\varepsilon v \omega^{2}}{c^{2}}-k_{r}^{2}}} .
\end{gathered}
$$

One has to be careful choosing the signs in Equation 4.20 to be sure to use

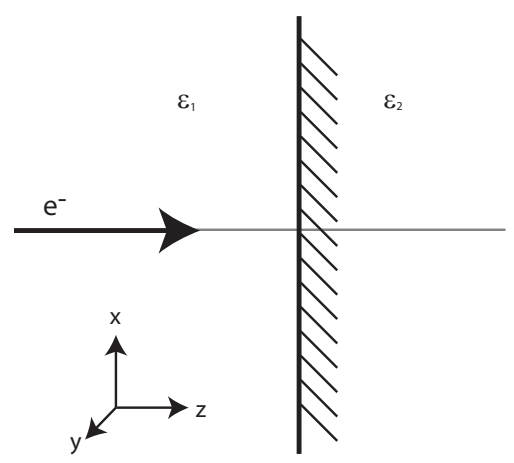

Figure 4.3: A charge moving along the $z$-direction across an interface between two media with dielectric constant $\varepsilon_{1}$ and $\varepsilon_{2}$.

waves propagating away from the interface, therefore for $z>0$ the upper signs and for $z<0$ the lower signs should be used. The coefficients $a_{1}$ and $a_{2}$ for the first and second medium can be derived from the boundary conditions for the field perpendicular and parallel to the interface $(z=0)$ which in our notation reads

$$
\begin{gathered}
\varepsilon_{1} E_{z}^{(1)}=\varepsilon_{2} E_{z}^{(2)}, \\
\mathbf{k}_{\mathbf{r}} \cdot \mathbf{E}_{r}^{(1)}=\mathbf{k}_{\mathbf{r}} \cdot \mathbf{E}_{r}^{(2)} .
\end{gathered}
$$


Solving this set of equations for $a_{1}$ using Equations 4.14, 4.13, 4.20 and 4.19 leads to the following expression

$$
a_{1}=\frac{\frac{\mathrm{i} q}{\omega \varepsilon_{0}} \frac{v}{c} \frac{k_{r}^{2} c^{2}}{\omega^{2}} \frac{\varepsilon_{2}-\varepsilon_{1}}{\varepsilon_{1}}\left(1-\frac{v^{2}}{c^{2}} \varepsilon_{1}+\frac{v}{c} \sqrt{\varepsilon_{2}-\frac{k_{r}^{2} c^{2}}{\omega^{2}}}\right)}{\left(1-\frac{v^{2}}{c^{2}} \varepsilon_{1}+\frac{k_{r}^{2} v^{2}}{\omega^{2}}\right)\left(1+\frac{v}{c} \sqrt{\varepsilon_{2}-\frac{k_{r}^{2} c^{2}}{\omega^{2}}}\right)\left(\varepsilon_{1} \sqrt{\varepsilon_{2}-\frac{k_{r}^{2} c^{2}}{\omega^{2}}}+\varepsilon_{2} \sqrt{\varepsilon_{1}-\frac{k_{r}^{2} c^{2}}{\omega^{2}}}\right)} .
$$

This equation, substituted in Equations 4.20 and 4.19 completely describes the transition radiation in the half-space $z<0$. These results are plane wave expansions of the transition radiation field and are therefore functions of $k_{r}$.

\subsubsection{Dispersion diagrams for the transition radiation}

In this section we will describe more specifically the situation in which an electron moves through the interface between vacuum and gold. For the numerical evaluation we use a measured value of the dielectric constant of gold [10]. The expressions for the fields $E_{z}$ and $E_{r}$ are functions of the frequency $\omega$ and the parallel wave vector $k_{r}$. In Figure 4.4 the amplitude of the electric fields is plotted as a function of $\omega$ and $k_{r}$ in a dispersion diagram for two different values of the distance $z$ above the interface. For distances high above the surface the field is mostly located above the light line $(z=10 \mu \mathrm{m}$, Figures $4.4 \mathrm{a}, \mathrm{b})$. This is fully expected since at this height all evanescent modes have decayed and only propagating light is present. At very short distances above the surface $(z=1 \mathrm{~nm}$, the two top figures) the most conspicuous feature is the sharp maximum that lies close to the light line. This maximum behaves like a surface bound mode as it drops as a function of altitude. In the next section we will see that this turns out to be the surface plasmon polariton (SPP) mode. At larger wavevectors, far below the light line, the values of $\left|E_{z}\right|$ and $\left|E_{z}\right|$ clearly have a non-negligible value (see also the cross section in Figure 4.5). The 'waves' associated with these large wavevectors are not surface plasmon polaritons or light in one of the media. They are essential in fulfilling the boundary condition on the surface when the highly localized field of the electron passes through the interface. These type of 'waves' were also noticed in theoretical work on dipoles above metallic surfaces [21] in which context they were named lossy surface waves. These waves were also discussed in section 2.5. 

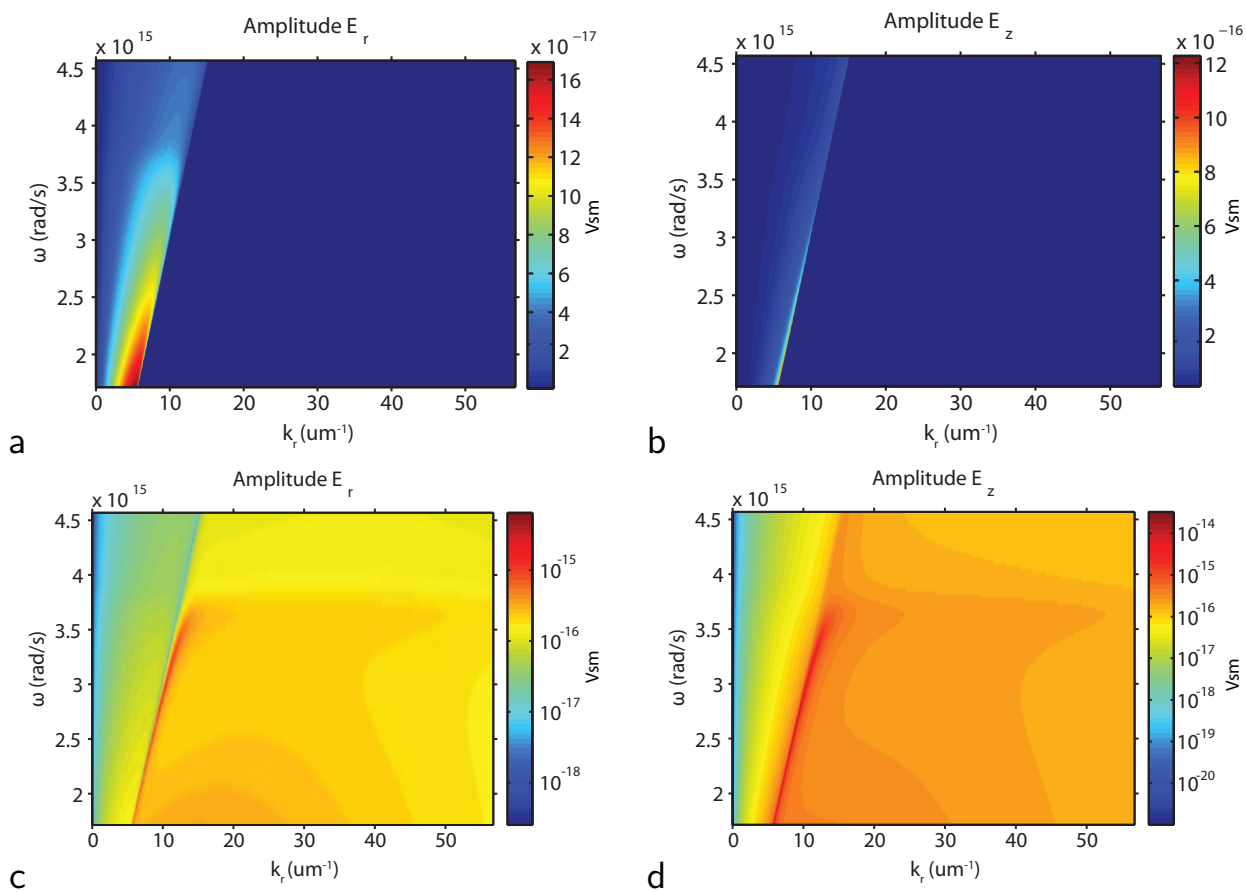

Figure 4.4: The amplitude of the electric field as a function of the parallel momentum $k_{r}$ and the frequency $\left.\omega . a, b\right)$ at a distance of $10 \mu \mathrm{m}$ above the interface, $c, d)$ at $1 \mathrm{~nm}$ above the surface. The left figures are for the field components parallel to the interface $\left(E_{r}\right)$, the figures on the right side are for the perpendicular components of the field $\left(E_{z}\right)$ 

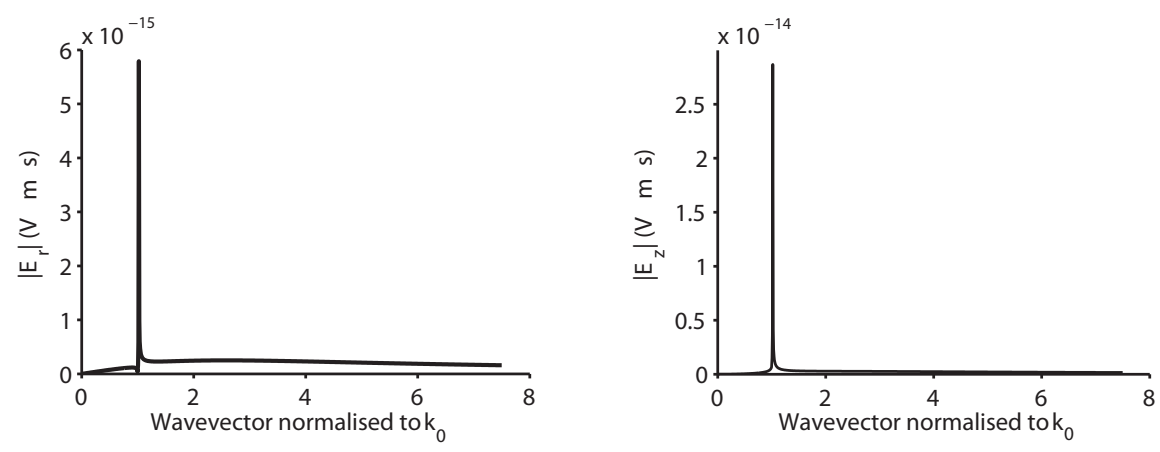

Figure 4.5: Cross sections of dispersion diagrams at $1 \mathrm{~nm}$ above the surface for a wavelength of $800 \mathrm{~nm}$. a) $E_{r}$, b) $E_{z}$. Clearly visible is the resonance of the surface plasmon

\subsubsection{The expression for transition radiation}

More insight in the physics of transition radiation is obtained by taking a closer look at Equation 4.23. We first consider the poles of the equation. The pole introduced by the third term in the denominator in 4.23 gives rise to the SPP contribution. This term is identical to the denominator in the Fresnel reflection coefficient for P-polarized light [12] and its dispersion is well known to describe the dispersion of SPPs. The two other terms in the denominator cause poles as well. As it turns out, both poles have the same dispersion as the Cherenkov radiation (see Section 4.1). These poles describe the Cherenkov radiation generated in the upper and lower half space.

It is important to realize that we are dealing with a forced solution of the Maxwell equations since the position and speed of the charge are fully specified. The homogenous solutions of the system are propagating plane waves in both media and the surface plasmons polaritons at the interface. The solution in Equation 4.23 is a solution of the inhomogeneous system. When the driving field - the moving electron - overlaps well with one of the homogeneous solutions of the system, for instance an surface plasmon polariton mode, it is intuitively clear that this mode will be efficiently excited. This explains the high field amplitudes at the surface plasmon dispersion curve in Figure 4.4. The large wavevector contribution - the lossy 
surface waves - are no solution of the homogenous system and therefore they will decay quickly in space.

The concept of the matching boundary conditions and specifically the matching of parallel momentum gives us a conceptual picture of what happens in more complicated structures. The moving electron being a point source will in a plane wave expansion have an infinitely broad distribution of wavevectors. Consequently, the boundary mismatch between the field of the electron above and below the surface will in general have a broad distribution in k-space. In any structure transition radiation fields will be generated that enable the boundary conditions to be fulfilled. In the system studied until now we can distinguish 3 different regimes according to the size of the wavevector. The first regime is that of plane waves described by $k_{r}<\sqrt{\varepsilon} \frac{\omega}{c}$. To match the boundary condition for these smallest wavevectors, plane waves have to be generated. In the region in k-space just below the light line surface plasmon polaritons are 'used' to fulfill the boundary conditions. To satisfy the conditions for the field close to the electron, the wavevectors with $k_{r}>\sqrt{\varepsilon} \frac{\omega}{c}$ are needed. These are the lossy surface waves described before. We can now understand that using the term 'wave' for these is perhaps somewhat misleading as our wave-like interpretation originates from that fact that we derived the transition radiation field as a plane wave expansion. They mainly represent the highly localized field of the electron. This contribution can however be scattered, for instance in structures where the dielectric constant varies rapidly in space.

\subsection{Transition radiation in real space}

To further understand the transition radiation microscopy technique we need to know the field in real space. Especially the excitation of surface waves by the moving electron is of interest since these surface waves can scatter at plasmonic structures on the surface. In Section 4.2 we obtained Equation 4.23 describing the electric field of transition radiation as a plane wave expansion. Deriving the electric field in real space implies performing a double inverse Fourier transform of this equation in the $k_{x}$ and $k_{y}$ direction. For the parallel component of the electric field this equates:

$$
E_{r}(r)=\iint_{-\infty}^{\infty} E_{r}\left(k_{r}\right) e^{\mathrm{i}\left(k_{x} x+k_{y} y\right)} d k_{x} d k_{y}
$$


where

$$
E_{r}\left(k_{r}\right)=\frac{\frac{\mathrm{i} q}{\varepsilon \varepsilon_{0}} \frac{v}{c} \frac{k_{r} c^{2}}{\omega^{2}} \frac{\varepsilon_{2}-\varepsilon_{1}}{\varepsilon_{1}} \sqrt{\frac{\varepsilon_{1} \omega^{2}}{c^{2}}-k_{r}^{2}} \mathrm{e}^{\mathrm{i} z \sqrt{\frac{\varepsilon_{1} \omega^{2}}{c^{2}}-k_{r}^{2}}\left(1-\frac{v^{2}}{c^{2}} \varepsilon_{1}+\frac{v}{c} \sqrt{\varepsilon_{2}-\frac{k_{r}^{2} c^{2}}{\omega^{2}}}\right)}}{\left(1-\frac{v^{2}}{c^{2}} \varepsilon_{1}+\frac{k_{r}^{2} v^{2}}{\omega^{2}}\right)\left(1-\frac{v}{c} \sqrt{\varepsilon_{2}-\frac{k_{r}^{2} c^{2}}{\omega^{2}}}\right)\left(\varepsilon_{1} \sqrt{\varepsilon_{2}-\frac{k_{r}^{2} c^{2}}{\omega^{2}}}+\varepsilon_{2} \sqrt{\varepsilon_{1}-\frac{k_{r}^{2} c^{2}}{\omega^{2}}}\right)}
$$

with $k_{r}^{2}=k_{x}^{2}+k_{y}^{2}$. For the perpendicular component of the field a similar expression can be derived. Performing the Fourier integral analytically is very challenging. A comparable problem, the field of a dipole radiating above a surface, has not been solved analytically yet, which makes us believe that a fully analytical solution of our problem is not likely to be obtained. We can find part of the solution analytically however as we will show. For the complete solution we will perform the integral numerically.

\subsubsection{Numerical solution of the Fourier integral}

Making use of the rotational symmetry of the problem, the two fold integral of Equation 4.25 can be shown to reducible to a single integral that is equivalent to a Fourier-Bessel integral. The field then equates:

$$
E_{r}(r)=2 \pi \int_{0}^{\infty} E_{r}\left(k_{r}\right) k_{r} J_{0}\left(k_{r} r\right) d k_{r}
$$

where $J_{0}$ is the zeroth order Bessel function of the first kind. As discussed before the value of $E_{r}\left(k_{r}\right)$ has a non negligible contribution for large k values (although $\lim _{k_{r} \rightarrow \infty} E_{r}\left(k_{r}\right)=0$ ). To perform the integral numerically we introduce an smooth cutoff in reciprocal space at $k_{\max }$. This automatically means that the result for distances below $r=\frac{2 \pi}{k_{\max }}$ will be incorrect. We have to note here that there are physical reasons that at these small distances our model is no longer valid as well; in the regime described by these large wavevectors spatial dispersion plays a role and this is not included in our model. A complication in performing the integral numerically arises as the Bessel function $J_{0}\left(k_{r} r\right)$ for large values of $r$ becomes a rapidly oscillating function. Using a quadrature method the integral was found to converge independently of the cutoff value $k_{\max }$ chosen in the range between $100 \mathrm{~nm}$ and $5 \mu \mathrm{m}$. The results are shown in Figure 4.7. 


\subsubsection{Analytical solution of the Fourier integral}

Although a complete analytic solution will not be derived here we will show that it is possible to find an analytic expression for the contribution of the poles of Equation 4.25. The most feasible way of solving integral 4.24 is probably by performing a contour integral [27]. Firstly we will rewrite the integral, using some useful properties of the Bessel function $J_{0}$ (see for instance [20]),

$$
E_{r}(r)=\pi \int_{-\infty}^{\infty} E_{r}\left(k_{r}\right) k_{r} H_{0}\left(k_{r} r\right) d k_{r}
$$

where $H_{0}$ is the zero order Hankel function of the first kind. In Figure 4.6 the path of the contour integral we want to perform is shown. As usual the integral over the contour for the half circle will not contribute to the integral. There are however 3 poles and 3 branch-cuts within the plane all of which have a contribution to the integral [26] therefore the field in real space will be

$$
E_{r}(r)=E_{c h 1}+E_{c h 1}+E_{s p}+\sum \int_{\Gamma_{n}} f\left(k_{r}\right) d k_{r}
$$

where $E_{c h 1}, E_{c h 1}$ and $E_{s p}$ are the contributions of the poles to the contour integral and the integrals over the paths $\Gamma_{n}$ are the contributions arising from the integrals around the branch cuts. We will not evaluate the contribution of the branch cut integrals, but the contribution of the poles can be determined by determining their residues. The positions of the poles are

$$
\begin{aligned}
& k_{c h 1}= \pm k_{0} \sqrt{\varepsilon_{1}-\frac{c^{2}}{v^{2}}}, \\
& k_{c h 2}= \pm k_{0} \sqrt{\varepsilon_{2}-\frac{c^{2}}{v^{2}}} \text { and } \\
& k_{s p}= \pm k_{0} \sqrt{\frac{\varepsilon_{2} \varepsilon_{1}}{\varepsilon_{1}+e_{2}}}
\end{aligned}
$$

with $k_{0}=\omega / c$. The first two poles originate from the Cherenkov terms the third is the surface plasmon polariton. The contribution of the poles to the integral is in all cases of the form

$$
E_{\text {pole }}=\beta_{\text {pole }} H_{0}\left(k_{\text {pole }} r\right)
$$




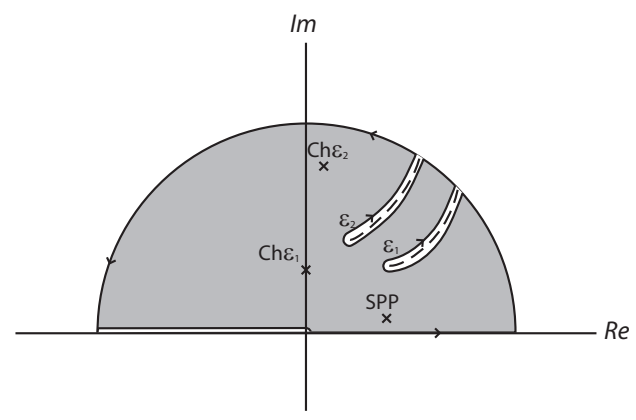

Figure 4.6: Schematic of the paths of the contour integration in the complex plane of $k_{r}$. The crosses indicate the positions of the poles. The two branchcuts are indicated with the dashed lines.

where $\beta_{\text {pole }}$ is a complex coefficient that is an elaborate function of the electron velocity and the dielectric constants of both media. We give here only the result for the surface plasmon pole on the surface $(\mathrm{z}=0)$

$$
E_{s p}=\frac{2 q v \pi^{2} \omega}{\varepsilon_{0} c^{3}} \frac{\frac{\left(\varepsilon_{1} \varepsilon_{2}\right)^{\frac{3}{2}}}{\left(\varepsilon_{1}+\varepsilon_{2}\right)^{\frac{5}{2}}}\left(1-\frac{v^{2}}{c^{2}}+\frac{v}{c} \sqrt{\frac{\varepsilon_{2}^{2}}{\varepsilon_{1}+\varepsilon_{2}}}\right)}{\left(1-\frac{v^{2}}{c^{2}} \frac{\varepsilon_{1}^{2}}{\varepsilon_{1}+\varepsilon_{2}}\right)\left(1+\frac{v}{c} \sqrt{\frac{\varepsilon_{2}^{2}}{\varepsilon_{1}+\varepsilon_{2}}}\right)} H_{0}\left(k_{s p} r\right) .
$$

In analogy with the work by Lalanne et al. and Gravel et al. $[24,26]$ we assume that in this way we have isolated the contribution of the surface plasmon polariton. Strictly speaking the remaining integrals for the branch cuts could completely change the final result. To verify this we will compare the contribution of the poles with the results from the numerical integration.

\subsubsection{Surface waves generated by transition radiation}

In Figure 4.7 the results of the analytic and numerical approach are plotted for 6 different wavelengths. As can be seen the result of the numerical calculation (dots) and the contribution of the SPP pole (dashed line) overlap very well, especially in the region $r>\lambda / 2$. In principle the contribution of the two other poles to the result should lead to an even more accurate description. Strangely enough this is not the case as the numerical results 
deviate considerably from the red line. In this regime however the contribution of the branch cut integrals is expected to be considerable as well, as these describe the evanescent field of the moving electron. This is the large wavevector contribution discussed in the end of section 4.2.2. Notice that for the longest wavelength $(905 \mathrm{~nm})$ the difference between the numerical results and the SPP pole is an oscillating function. This difference remains visible up to a distance of $3 \mu \mathrm{m}$. We believe that this difference is the same surface wave as Lalanne and Co-workers noticed (see Section 2.5). From the presented results we conclude that the field on the surface is very well described by surface plasmon polaritons at distances larger than $\lambda / 2$. Below $\lambda / 2$ we have numerical results that give us reliable values for the fields at the surface up to distances of $100 \mathrm{~nm}$ to the source.

\subsection{Comparison of the transition radiation with the field of a dipole}

Since the electron acts as a very local source one might wonder whether the field generated is similar to the field generated by an oscillating dipole close to the surface. First of all this could be of interest in a conceptual way. Radiation from a dipole source is something many people in the nano-optics field are more familiar with than transition radiation. Secondly, a range of applications exists in which dipoles close to a plasmonic surface play an important role. Since it is experimentally difficult to know where dipoles are located on the surface, it would be useful if the transition radiation technique -in which the electron beam can be pointed at any arbitrary position- is capable of measuring similar properties. A comparison between the radiation pattern of a oscillating dipole oriented perpendicular to the surface and the radiation pattern of the transition radiation is therefore useful.

The power of the transition radiation radiated in the far-field ([66] p.30) is

$$
W_{q}(\theta) \sim \sin ^{2}(\theta)\left|\frac{1+r}{1-\frac{v}{c} \cos (\theta)}-\frac{\sqrt{1-r^{2}}}{n-n^{2} \frac{v}{c} \sqrt{1-\frac{\sin ^{2}(\theta)}{n^{2}}}}\right|^{2},
$$

where $n$ is the refractive index of the medium, $r$ the Fresnel reflection coefficient for p-polarized light, $\theta$ the angle with respect to the surface 

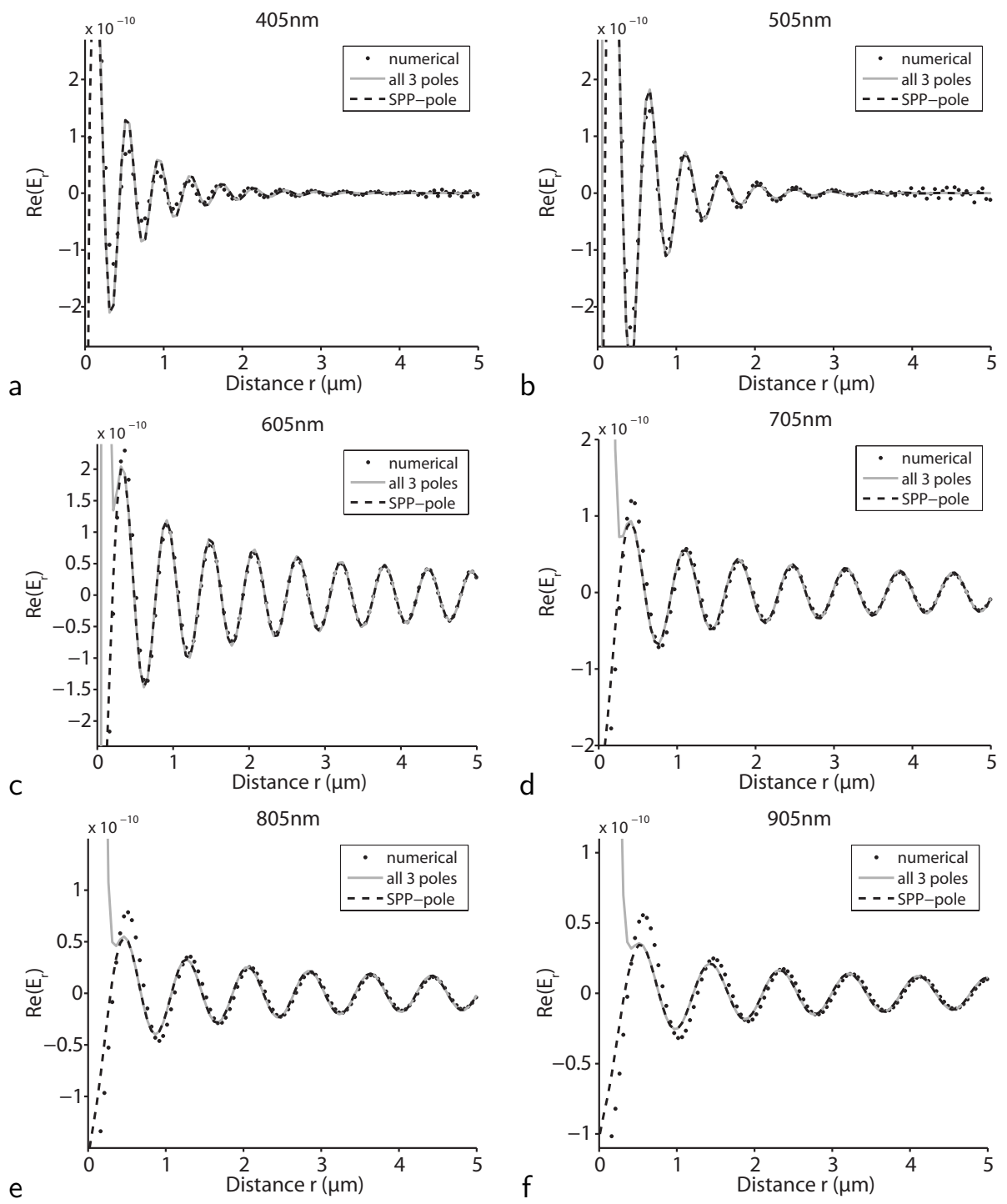

Figure 4.7: Theoretical calculations of the parallel component of the transition radiation on the surface for 6 wavelengths (405, 505, 605, 705, 805 and $905 \mathrm{~nm}$ ). Numerical results (dotted-line), SPP-pole contribution (dashedline), Contribution of all 3 poles (red-continuous-line). 
normal. The power radiated in the far-field for a dipole in vacuum, oriented perpendicular to a surface is given by ([12] p.350):

$$
W_{d i p} \sim \sin ^{2}(\theta)\left|\mathrm{e}^{-\mathrm{i} z_{0} n k_{0} \cos (\theta)}+r \mathrm{e}^{\mathrm{i} z_{0} n k_{0} \cos (\theta)}\right|^{2},
$$

with $k_{0}=\frac{w}{c}$ and $z_{0}$ the hight of the dipole above the surface. When we take the limit of $z<<\frac{2 \pi}{k_{0}}$ in the case of the dipole and $\frac{v}{c}<<1$ in case of the transition radiation the radiation patterns are identical for $r \approx 1$. The radiation pattern of the transition radiation can therefore be considered the same as the pattern of an dipole oriented with its dipole moment perpendicular to the interface placed much closer than a wavelength to the interface when the electron velocity is low and the interface is highly reflective. For gold at wavelengths above $600 \mathrm{~nm}$ this last condition is certainly satisfied.

The resemblance of the radiation patterns is a promising fact. The more interesting contribution of SPPs and lossy surface waves is however not described by them. A more thorough approach is is therefore worth some effort. The field of a dipole close to a surface can be derived using a Green tensor method. The parallel component of the electric field for a dipole with its dipole moment oriented in the $z$ direction is then given by $[12]$

$$
\begin{aligned}
E_{r, \text { dip }}= & \frac{i \mu_{\text {dip }}}{8 \pi^{2} \varepsilon \varepsilon_{0}} \mathbf{k}_{\mathbf{r}}\left(\mp e^{ \pm i \sqrt{\frac{\omega^{2}}{c^{2}} \varepsilon-k_{r}^{2}}\left(z-z_{0}\right)}+\right. \\
& \left.\frac{\varepsilon_{1} \sqrt{\varepsilon_{2}-\frac{k_{r}^{2} c^{2}}{\omega^{2}}}-\varepsilon_{2} \sqrt{\varepsilon_{1}-\frac{k_{r}^{2} c^{2}}{\omega^{2}}}}{\varepsilon_{1} \sqrt{\varepsilon_{2}-\frac{k_{r}^{2} c^{2}}{\omega^{2}}}+\varepsilon_{2} \sqrt{\varepsilon_{1}-\frac{k_{r}^{2} c^{2}}{\omega^{2}}}} e^{i \sqrt{\frac{\omega^{2}}{c^{2}} \varepsilon-k_{r}^{2}}\left(z+z_{0}\right)}\right) .
\end{aligned}
$$

Here $\mu_{\text {dip }}$ is the dipole transition moment, $z_{0}$ is the height of the dipole above the surface and the upper signs apply for $z>z_{0}$ and the lower signs apply for $z<z_{0}$. This equation should be compared to 4.25. Most importantly it has same denominator as is present in Equation 4.25. The pole introduced by $\varepsilon_{1} \sqrt{\varepsilon_{2}-\frac{k_{r}^{2} c^{2}}{\omega^{2}}}+\varepsilon_{2} \sqrt{\varepsilon_{1}-\frac{k_{r}^{2} c^{2}}{\omega^{2}}}=0$ is also in this case related with excitation of surface plasmons. With a z-oriented dipole efficient excitation of SPPs is therefore expected as well. Of course no Cherenkov radiation term is expected in the expression for the dipole radiation, which explains the absence of any other poles. The lossy surface waves are also 
present in radiation of the dipole [21]. Their amplitude is mathematically not identical to their amplitude in the case of transition radiation. In both cases however the behavior under the light line away from the SPP pole is smooth and slowly converging to 0 for $k_{r} \rightarrow \infty$. To conclude, the transition radiation and the radiation of a dipole on the surface most definitely have many features in common and the analogy between both is a useful one, both are however not equivalent.

\subsection{Transition radiation for nano-optics}

The attractive part of the use of transition radiation as a microscopy tool in nano-optics is the extremely high resolution with which an electron beam can be focused and positioned. In a typical electron microscope this resolution can be smaller than $10 \mathrm{~nm}$. The scale at which optical information is acquired, depends on the length scales on which the optical properties of the structure under investigation vary. In principle when the structure has features that are abrupt they are capable of scattering the large wavevectors produced around the impact position of the electron. Therefore also the resolution of this technique will be close to $10 \mathrm{~nm}$. Another way of picturing the high resolution of the transition radiation microscopy, is that the near field components of the electron can be scattered by the structure.

The analytical method to derive the electromagnetic fields of transition radiation on a plain surface was chosen because it gives the best insight into the physical processes that play a role. Numerical techniques are available that are more suitable for calculating transition radiation on metal surfaces with plasmonic structures. The boundary element method (BEM) is the current state of the art technique for performing such calculations [67, 68]. This method is however up to now only practical for cylindrical symmetric and 2 dimensional systems [69].

In the end of Section 4.2.2 the boundary conditions on the interface were employed as a tool to intuitively understand the generation of transition radiation. In that section the emphasis was on the boundary conditions in k-space. In many nano-optic structures it might be more suitable to view the same effect in real space, where the continuity of the perpendicular component of $\mathbf{D}$ and the parallel component of $\mathbf{E}$ across the surface are the boundary condition. This way one can understand that the moving electron is also capable of exciting for instance localized plasmonic resonances. In 
this thesis we will investigate small holes in a plain gold layer. When considering transition radiation as a microscopy tool on such structures we can consider two regimes. When the electron passes through the interface far away from the hole, we can consider the hole as a small perturbation with respect to the situation discussed in this chapter, an infinitely large plain surface. When the electron passes through the surface close to the hole we can no longer consider the hole as a small perturbation. A discussion involving the more practical aspects that play a role in the interpretation of transition radiation microscopy data is given in Chapter 5 . 


\section{Chapter 5}

\section{Transition radiation microscopy}

Practical information on the use of transition radiation as a microscopy tool is discussed. The principle of operation is explained. Technical specifications of the optical system and the electron microscope are given.

\subsection{Introduction}

The past 20 years the field of nano-photonics has been a rapidly growing field. The spectacular improvements in nano-fabrication techniques went hand in hand with the development of new methods to study optical systems beyond the diffraction limit. Techniques such as near-field scanning optical microscopy [70] and specialized fluorescence microscopy techniques [71] are nowadays well established experimental techniques. In nano-plasmonics, apart from the all optical techniques available in nanophotonics, various techniques have been developed that use electron beams to acquire optical information. These techniques all employ the high spatial resolution that can be obtained with electron microscopy. Although techniques to study surface plasmon polaritons with electron beams have been available since the 1960s [72], only recently the value of these techniques for research on nanoplasmonic systems has become widely recognized in the plasmonics community $[4,73,74,75]$.

The measurement technique described in this chapter is based on the 
detection of light that is generated by the impact of a focused electron beam on a nanoplasmonic sample [76, 77]. This method of detection of electron beam induced light is historically referred to as cathodoluminescence. This term is however somewhat misleading because the light detected in these methods does not originate from the cathode and is not necessarily luminescence either. In the experiments described in this thesis the light generated is transition radiation (see Chapter 4), therefore throughout the rest of this thesis the term transition radiation will be used.

In this chapter the method of measuring transition radiation as used in this thesis will be described. Firstly there will be a compact general introduction into the basic principle of transition radiation microscopy for nano-optics. Second there will be a description of the used setup, and the experimental technique including some basic results.

\subsection{Principles of operation}

In the experiments described in this thesis the observed light is transition radiation. There are many other mechanisms by which optical radiation can be formed under impact of an electron beam, for instance thermal, fluorescent or phosphorescent processes [78], Cherenkov radiation and bremsstrahlung. In principle all of these processes can be used as a microscopy tool as described in this chapter. The samples investigated in this thesis have gold as their main constituent. For this material no fluorescent or phosphorescent processes are expected in the wavelength regime of interest. Thermal effects can be excluded based on the fact that even after long periods of measurement the samples under investigation show no thermal damage. Brehmstrahlung is under normal conditions in a SEM perpendicular incidence of the electron beam and non-relativistic electron velocities - much weaker than transition radiation [79]. Cherenkov radiation was shown in Chapter 4 to have a minor contribution but in all cases it is included in the models that we will use to interpret our experiments.

\subsection{Transition radiation microscopy}

The experimental technique is illustrated in figure 5.1. Two contributions of transition radiation generated by the electron beam to the detected light can be distinguished. Firstly there is a directly radiated part that emerges 


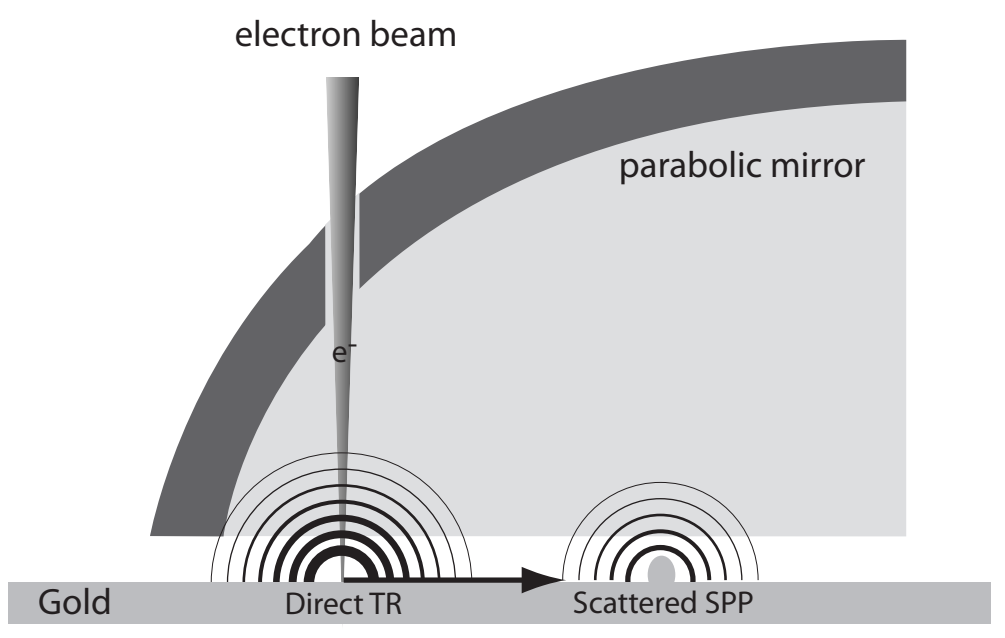

Figure 5.1: Schematic illustration of the measurement mechanism. At the position of the electron beam direct transition radiation (TR) is generated as well as surface waves (mostly surface plasmon polaritons). The SPPs can scatter to light at for instance a protrusion on the surface. Both radiating components are collected by the parabolic mirror. In this schematic illustration the distance between the position where the electron beam hits the sample and the protrusion where the SPP is scattered, is out of proportion with respect to the size of the parabolic mirror. In the actual configuration the collection spot of the parabolic mirror is much larger than the distance between electron beam and protrusion. 
from the point of electron impact. Secondly, a surface wave part that on a planar surface - will manifest as an outward traveling surface wave. At distances larger than the wavelength of the light this contribution is mainly due to surface plasmon polaritons excited by the electron beam. The generated surface waves can scatter on for instance a roughness on the surface and thus couple to free radiation that will be detected. The two contributions to the generated light are collected with a parabolic mirror and directed towards a spectrometer. The measured light is therefore the interference between the light generated directly by the electron beam and the light generated by the scattering of surface waves [80]. The small focus of the electron beam enables a highly localized excitation process, therefore the generated light is characteristic of the local properties of the sample at the position of the electron beam. By raster scanning the position of the electron beam over the sample a spectrum can be recorded at every position of the sample. In this experimental technique the exact position where the light is emitted is not known. The high resolution is achieved by using a local 'excitation' of the sample rather than a local detection.

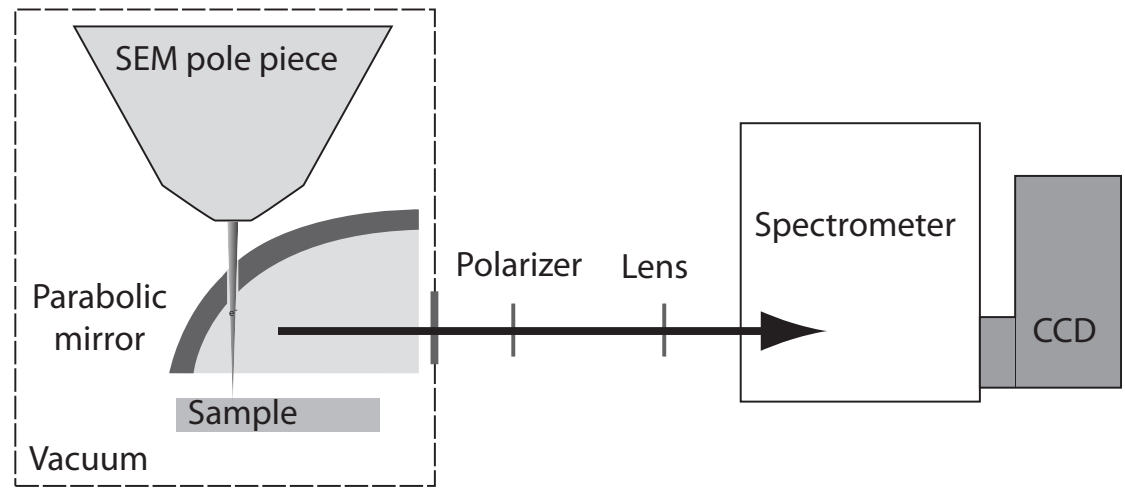

Figure 5.2: Schematic representation of the setup for the detection of electron beam-induced light. In the vacuum chamber of the SEM the sample is bombarded with a focused beam of electrons. The light generated is collected with a parabolic mirror. This mirror has a small hole in it, that allows the electron beam to pass through the mirror to the sample. The light collected by the parabolic mirror is directed through a vacuum window to spectrometer that is equipped with a CCD camera. 


\subsection{Instrumental information}

The setup used for the measurements is a FEI XL30 SFEG scanning electron microscope equipped with a cathodoluminescence system by Gatan. A parabolic mirror with a working distance of $1 \mathrm{~mm}$ is placed under the pole piece of the SEM. In the parabolic mirror there is a hole with a diameter of $1 \mathrm{~mm}$ to enable access of the electron beam to the sample. The spectrometer used is a $300 \mathrm{~nm} \mathrm{f/4.2} \mathrm{imaging} \mathrm{spectrometer} \mathrm{with} \mathrm{a} \mathrm{Prince-}$ ton Instruments Spec10:100 front illuminated liquid nitrogen cooled CCD. The dispersing grating used has 150 lines $/ \mathrm{mm}$ which results in a spectrum ranging in wavelength roughly from 400 to $950 \mathrm{~nm}$. The entrance slit is set to $2 \mathrm{~mm}$ to collect maximal signal, the corresponding spectral resolution is limited by the point spread function of the optical system and is estimated to be $10 \mathrm{~nm}$.

The measurements are done with settings of the SEM that maximize the amount of signal collected. Therefore the highest acceleration voltage of $30 \mathrm{kV}$ is used, which leads to an electron velocity of approximately a third of the velocity of light. Further settings of the microscope are chosen to maximize the beam current. According to the specifications of the microscope the corresponding beam current is approximately $40 \mathrm{nA}$. The beam waist at the point of the point of impact is $20 \mathrm{~nm}$.

The parabolic mirror collects light in $1.4 \pi$ steradians [81]. Because the parabolic mirror is asymmetric along the axis of the paraboloid, the optical system has a slight asymmetry in the angle-dependent collection efficiency along one axis.

In Figure 5.3a a typical spectrum measured on a plain surface of gold is shown. Since we know the transition radiation spectrum from theory we can compare the measured spectrum with the spectrum derived from theory. There are a few unknown factors that relate the experiment with the theory. Firstly the efficiency of the detection system, which includes the transmission of the lens system, the reflection at 5 mirrors, the efficiency of the grating and the quantum efficiency of the CCD of the spectrometer. Secondly the exact beam current is unknown. Using the beam current given by the specification of the SEM we calculate the detection efficiency of the optical system (see Figure 5.3b). The maximum efficiency we derive is $1.4 \%$. This efficiency is very reasonable considering the large amount of optics between sample and detector. We conclude from this that the experimental conditions are well controlled and that the derived expression 

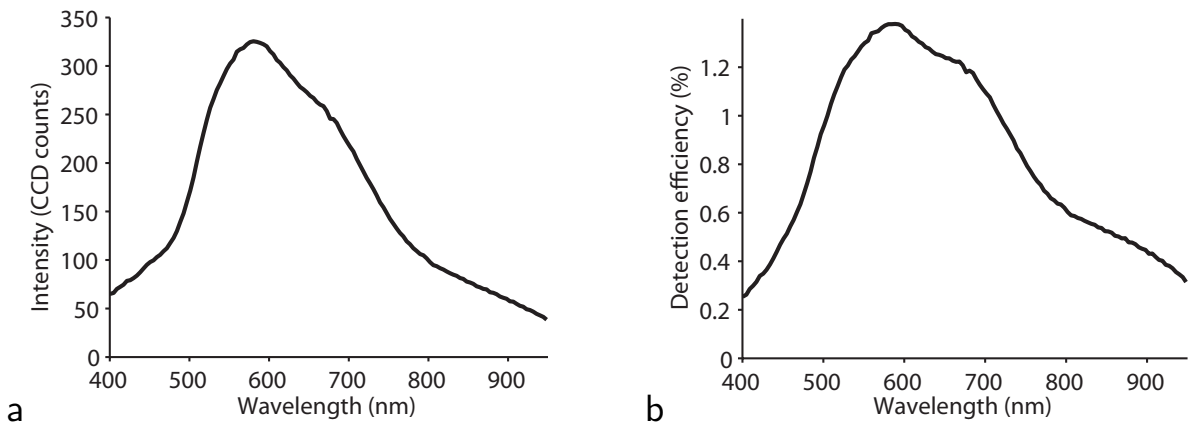

Figure 5.3: (a) Typical spectrum collected in measurement on a plain gold film. (b) Detection efficiency spectrum of the optical system of the experimental setup derived assuming a beam current of $40 \mathrm{nA}$. The efficiency curve resembles the known quantum efficiency spectrum of the CCD detector.

for the transition radiation gives an accurate description of the mechanism resulting in the observed light. 


\section{Chapter 6}

\section{Scattering of surface plasmon polaritons on single holes}

We investigate the scattering of surface plasmon polaritons on single cylindrical holes in metal layers. We use transition radiation microscopy to launch surface plasmon polaritons and measure the interference between the direct transition radiation and the scattered surface plasmon polariton. We propose a simple model describing the scattering properties of a hole by an effective dipole. A good qualitative match between model and experiment is found, allowing an estimate of the effective polarizability of a single hole.

\subsection{Introduction}

The scattering of surface plasmon polaritons (SPP) on subwavelength holes is a topic of great interest to the plasmonics community [82]. This is due mainly to the large interest in the extraordinary transmission phenomenon [1] (see also Section 2.6). It is generally accepted that the launching of SPPs by light incident on a hole or emanating from a hole and the subsequent scattering of these SPPs at neighboring holes is essential in describing this effect [25]. Experimental results on the scattering properties of SPPs are important as they could be used to verify and improve the proposed models. Experimental investigations of the scattering of SPPs to light by single holes 
and the launching of SPPs by light are however very challenging [41, 22].

In Chapter 4 we have shown that at distances larger than roughly $\lambda / 4$ the field on the surface of a metal, created by the passing of an electron is mainly due to the SPPs that are excited with the transition radiation microscopy technique. Within the wavelength range investigated, the contribution of lossy surface waves was shown to be small. We thus have a method to create a well-defined source of SPPs that can be used to study the scattering properties of subwavelength holes.

In this chapter we investigate the scattering of SPPs on single cylindrical holes in metal layers. We describe the experiment and discuss the results. Furthermore, a model is proposed describing the scattering properties of a hole by an effective dipole.

\subsection{Transition radiation near a single hole}

The sample under investigation is a cylindrically shaped aperture with a diameter of $260 \mathrm{~nm}$ in a $200 \mathrm{~nm}$ thick layer of gold on a silicon substrate. The measurement described in this chapter is a line scan of the electron beam from the center of the aperture outwards (see Figure 6.1). At each point along the line a spectrum is collected. Since in this chapter we are interested in the contribution of the scattered surface plasmon polariton we will discuss results obtained moving the electron beam further than 200 nm away from the aperture.

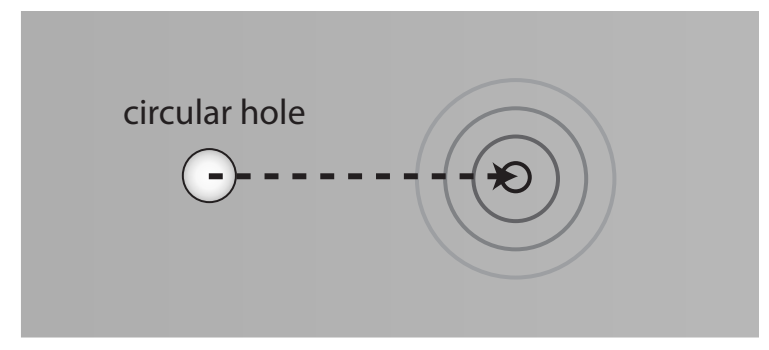

Figure 6.1: Schematic representation of the measurement performed. The electron beam was moved along a straight line from the center of the hole outwards. At every position along the line a spectrum is collected.

In Figures 6.2, 6.3 and 6.4 the results of the performed measurement are 
shown. In Figure 6.2 we plot the value $S(d, \lambda)=\left(I_{\text {det }}(d, \lambda)-I_{\text {plain }}(\lambda)\right) / I_{\text {plain }}(\lambda)$ where $I_{\text {det }}(d, \lambda)$ is the detected spectrum at a distance $\mathrm{d}$ from the center of the hole and $I_{\text {plain }}(\lambda)$ is the spectrum detected far from the aperture. The figure shows a normalized map of the spectrum as a function of the distance from the center of the hole. In the map of $S(d)$, fringes are visible as a function of distance that have roughly a period $\lambda / 2$. Cross sections of the detected intensity $I_{\text {det }}(d, \lambda)$ are shown for 4 different wavelengths in Figure 6.3. In this graph a high intensity peak near the edge of the aperture is visible. As this peak contains more contributions than the SPP alone, we do not consider it here. To get a better view on the fringes in Figure 6.4 the value $I_{d e t}(d, \lambda)-I_{\text {plain }}(\lambda)$ is plotted. Here one can observe that the period of the fringes is roughly $\lambda / 2$. The spacing between the minima and maxima in the graph obtained for $597 \mathrm{~nm}$ are indicated with arrows. Their spacing is 150, 120 and $120 \mathrm{~nm} \pm 20 \mathrm{~nm}$. A more detailed analysis of the fringes is difficult due to the low signal and low number of fringes observed. Figure 6.4 also shows that the fringes are placed on a background that decays as the distance from the hole increases.

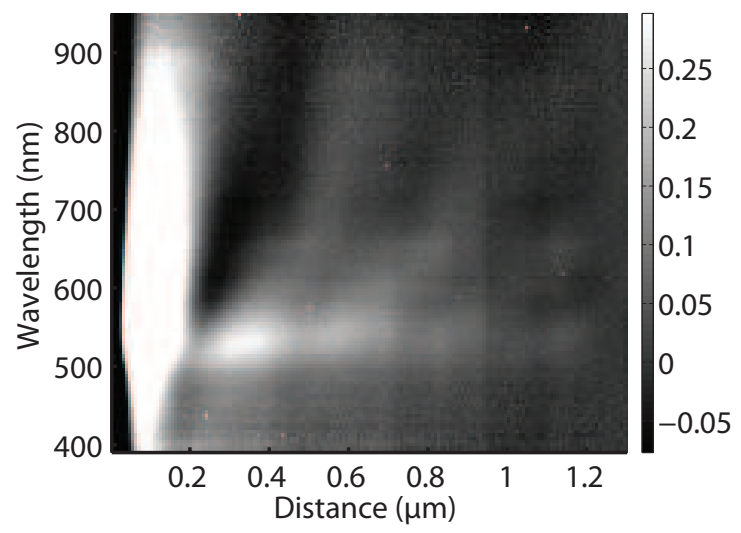

Figure 6.2: Map of $S(d, \lambda)=\left(I_{\text {det }}(d, \lambda)-I_{\text {plain }}(\lambda)\right) / I_{\text {plain }}(\lambda)$ where $I_{\text {det }}(d, \lambda)$ is the detected spectrum at each position and $I_{\text {plain }}(\lambda)$ is the spectrum collected on a plain gold surface. Fringes with a period equal to half the wavelength are visible as a function of the distance. To enhance the visibility of the fringes the color scale is saturated. 


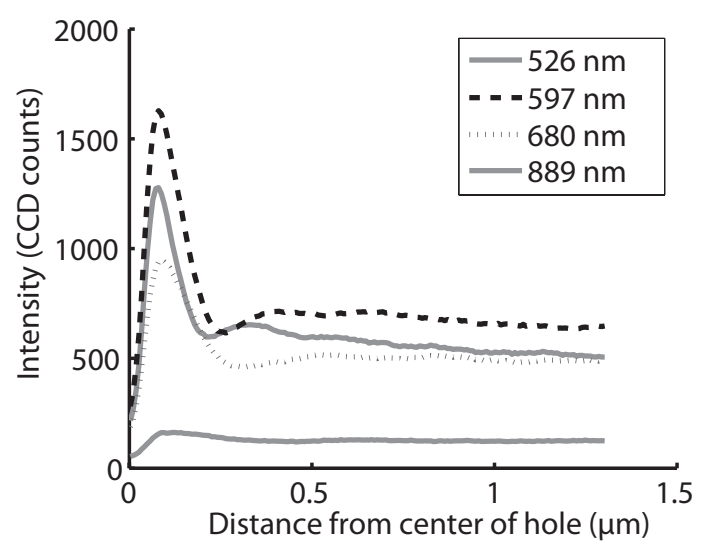

Figure 6.3: Intensity of the collected transition radiation as a function of distance to the center of the hole for 4 different wavelengths. The acquisition time for each data point was 2 seconds.

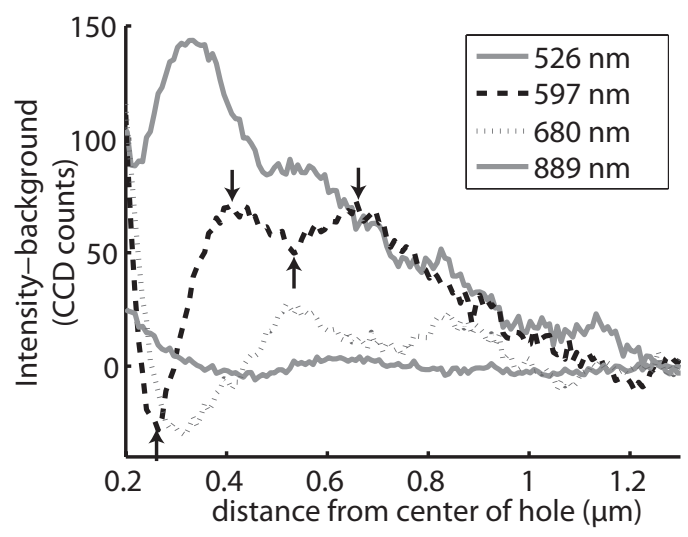

Figure 6.4: Intensity of the collected transition radiation as a function of distance to the center of the hole for 4 different wavelengths. The same data as 6.3 but with the transition radiation from a plain gold surface subtracted such that all graphs end at 0 CCD counts at $1.3 \mu \mathrm{m}$ distance. 


\subsection{The effective dipole scattering cross section}

We propose a simple model to interpret the results described in the previous section. In analogy with the results of Lalanne et al. [24] that showed good agreement between the surface plasmon polariton scattering properties of a slit and a line dipole oriented parallel to the surface, we model the small hole as a dipole. The induced effective dipole moment is $\mathbf{p}=\alpha(\omega) \mathbf{E}$, where $\alpha(\omega)$ is the polarizability of the hole. $\mathbf{E}$ is the electric field of the transition radiation at the position of the hole. We will use the fields on the surface as calculated in Chapter 4 as the driving field of the effective dipole, thereby assuming the hole to be a small perturbation on the unstructured surface. The effective dipole can be either oriented in the plane of the surface pointing towards the plasmon source (x-orientation) or oriented perpendicular to the surface (z-orientation). In the first case the dipole is driven by the longitudinal component of the SPP field, while in the second case it is driven by the transverse component of the SPP field. It is expected that $\alpha$ is frequency dependent as the scattering properties will be dependent on de dielectric constant contrast between the hole and vacuum. The intensity we detect is the far-field interference of the direct transition radiation with the radiation scattered by the effective dipole. We use the plane wave expansion of the expression for transition radiation obtained in chapter 4 and the plane wave expansion of the field of a dipolar light source on a surface [12]. We integrate over all the values of the inplane wavevectors $k_{x}$ and $k_{y}$ implicitly assuming that the parabolic mirror collects the light in $2 \pi$ steradians. Additionally we used the detection efficiency as derived in Section 5. In this model the polarizability $\alpha(\omega)$ is the only free parameter, the amplitude of which is tuned manually to obtain correspondence to the experimental results.

The results of this model are depicted in Figure 6.6 for a dipole oriented in the plane of the surface and perpendicular to the surface respectively. To enable a direct comparison with the experimental results depicted in Figure 6.4 , in both figures $I_{\text {det }}-I_{\text {plain }}$ is plotted. The polarizabilty chosen in these models is $2 \cdot 10^{-26} \mathrm{Cm}^{2} / \mathrm{V}$ for the z-oriented dipole and $1 \cdot 10^{-27} \mathrm{Cm}^{2} / \mathrm{V}$ for the x-oriented dipole independent of the wavelength. The polarizabilty can have a complex value. Varying the phase factor leads to changes in the phase of the fringe pattern that is observed in the figures, but not in the general decaying trend. Therefore the phase of $\alpha$ in both figures was set to zero. 


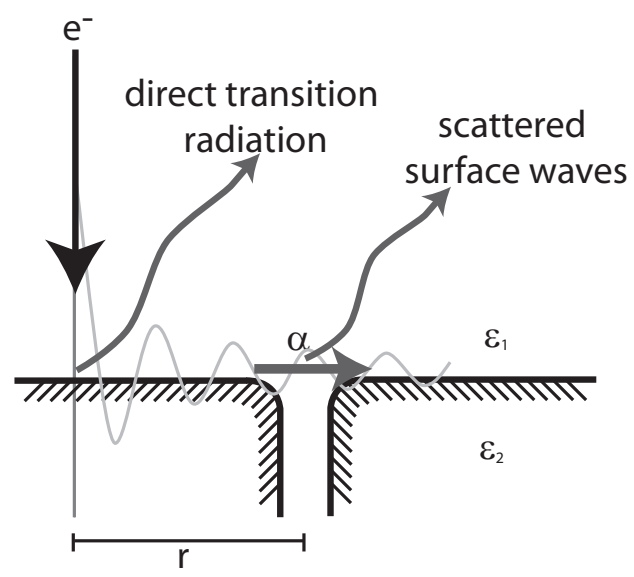

Figure 6.5: Schematic overview of the mechanism adopted in the model. At the position of the electron beam both direct transition radiation and a surface wave, consisting of mainly the surface plasmon polariton, are generated. The surface wave is scattered at the hole, this scattering is modeled by a dipole with polarizability $\alpha$ that is driven by the field of the SPP at the position of the hole.
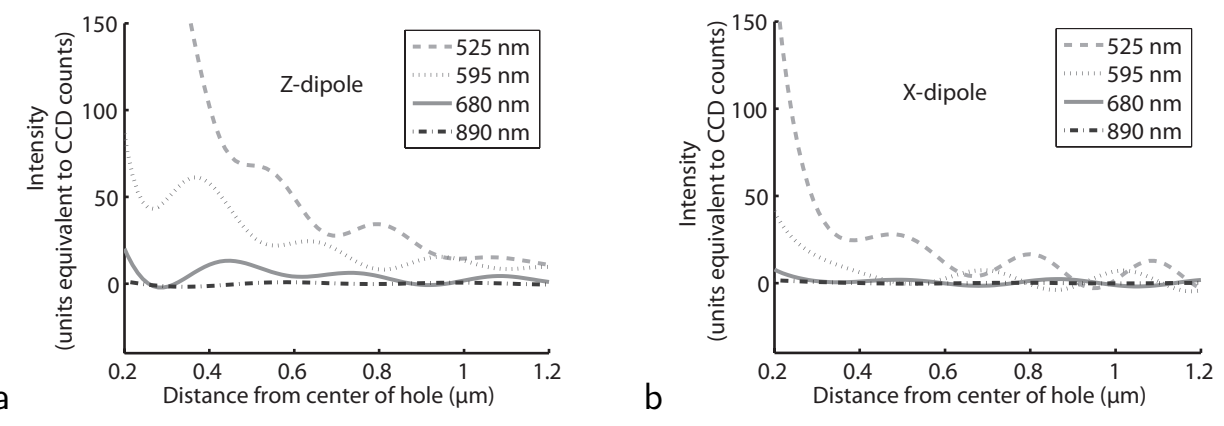

Figure 6.6: The distance dependence of the collected intensity for 4 different wavelengths assuming (a) a dipole oriented perpendicular to the surface with a polarizability $\alpha_{z}=2 \cdot 10^{-26} \mathrm{Cm}^{2} / V$. (b) A dipole oriented in the plane of the surface with its dipole moment pointing towards the hole and a polarizability $\alpha_{x}=1 \cdot 10^{-27} \mathrm{Cm}^{2} / \mathrm{V}$ 
The model describes the basic characteristics observed in the measurement. Both in the measurement and the model oscillations with a period equal to roughly half the wavelength are visible on a decaying background. Additionally the relative strengths of the curves for different wavelengths are correct; in both the measurement and the model the highest intensity is observed for the wavelengths around $525 \mathrm{~nm}$ while the lowest signals are observed at $890 \mathrm{~nm}$. Most importantly it is possible to obtain an estimate of the effective polarizability of the aperture. By changing the value of $\alpha$ such that the intensities in the model are comparable to the measured value (in the range of 10 to $150 \mathrm{CCD}$ counts) we obtain a value of $\alpha \approx 10^{-27}-10^{-26} \mathrm{Cm}^{2} / \mathrm{V}$.

Based on the model and the experimental results the current understanding of the results is as follows. The light scattered at the hole and the direct transition radiation both have different radiation patterns. The detected signal can be considered to consist of a part where both radiation patterns overlap and a part where only light from either the direct transition radiation or the dipole is observed. In the direction where the radiation patterns overlap the interference of the two contributions leads to an oscillation on the measured amplitude as a function of distance. The period of this oscillation is dependent on the exact radiation patterns and the angles collected by the optical system [80]. The directions in which the radiation patterns don't overlap lead to an offset contribution in the measured signal which gives rise to the increasing background as the distance to the hole increases.

\subsection{Discussion}

Our model successfully describes the observed features in the experiment and yields an estimate of the effective polarizability of a hole. This result shows that we have a good understanding of the physics that plays a role in our experiment. This is highly promising, but a better match between measurement and theory would be valuable. The ultimate goal, obtaining an accurate value for the effective polarizability is worth the effort. Several improvements can be suggested for both the model and the experiment. Further improvement of the experiment should be focused mainly on improving signal to noise, for instance by improvements of the setup leading to a larger detection efficiency. Alternatively longer integration times could 
be used. The most prominent improvements of the model could be done by taking the acceptance angles of the mirror into account. Last but not least, it would be worthwhile to check in a separate experiment or theory what the orientation of the effective dipole is depending on hole size and shape. 


\section{Chapter 7}

\section{Local investigation of rectangular holes}

The optical properties of rectangular subwavelength holes are studied with transition radiation microscopy. Along the edges of the hole, high peaks in the generated transition radiation are observed. The dependence of the spectrum of these peaks on the dimensions of the hole is investigated systematically. It is proposed that the measurement technique probes the scattering strength of the hole and that this strength depends on the existence of waveguide modes in the hole.

Subwavelength apertures play a prominent role in nano-plasmonics because of their ability to couple far-field radiation to surface plasmon polaritons and as such have been the subject of intensive study [1, 25, 82]. To gain insight into the electromagnetic fields around the aperture, numerical techniques are available $[83,56]$. Experimentally however, it is very challenging to measure the fields in and around apertures in the visible regime. Some results have been obtained with near-field scanning probe techniques $[84,40]$, but convolution with the probe collection efficiency limits the resolution that is obtained such that the effect of hole shape is difficult to investigate.

Transition radiation microscopy has a superior resolution and can be used to gain insight into the physics very close to these apertures [2]. In this chapter we will present results of measurements on subwavelength holes in gold in the near-field regime; when the distance to the hole is smaller than $\lambda / 2$. We will present results of measurements on a rectangular hole and a 
systematic analysis of the influence of hole shape.

\subsection{Rectangular holes}

The structures under investigation in this chapter are rectangular holes fabricated with focused ion beam (FIB) milling in a $200 \mathrm{~nm}$ thick layer of gold on a silicon substrate (see Figure 7.1). The actual dimensions of these small holes are difficult to determine. From scanning electron microscopy images (see for instance Figure 9.4) it is visible that the holes are slightly tapered and that the edges of the holes have a smooth curvature. This was confirmed by atomic force microscope measurements. Due to the taper and the radius of curvature, it is hard to define the location of the edge from AFM data. Therefore, the dimensions of the holes as quoted in the presentation of the results in this chapter, are the original design parameters. The deviation from design parameters is small, 10 to $25 \mathrm{~nm}$, and is caused mainly by the spot size of the focused ion beam and a scaling factor that is chosen in the FIB milling. Since these deviations are small and systematical, the design parameters give an accurate description of the hole size.

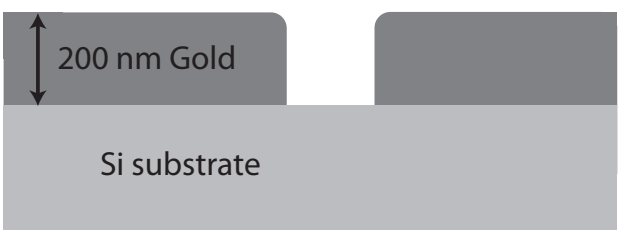

Figure 7.1: Schematic side view of the system under investigation. A subwavelength hole in a $200 \mathrm{~nm}$ thick layer of gold on a silicon substrate

\subsection{Transition radiation microscopy on a single rectangular hole}

The results of a transition radiation microscopy measurement on a rectangular hole with dimensions $100 \times 260 \mathrm{~nm}$ is presented in Figures 7.2 to 7.4. Figure $7.2 \mathrm{a}$ shows the intensity of light collected while raster scanning the 
electron beam over the sample. In this image the color indicates the total number of integrated counts collected by the detector for each position of the electron beam. Two cross-sections of this image are shown in Figure 7.3a. Both cross-sections run through the center of the hole, one along its short axis and one along its long axis. Simultaneously with the optical signal a SEM signal is collected, depicted in Figure 7.4. In the SEM image 3 positions are indicated by letters A,B and C. Spectra collected at these positions are given in the graph in Figure $7.3 \mathrm{~b}$. Figure $7.2 \mathrm{~b}$ shows the average wavelength at every pixel position. This data is constructed from the spectra collected at every pixel position by taking the weighted average of the corresponding spectrum. Because the spectra in these measurements always show only one maximum an image of the average spectrum gives a good qualitative insight into the measured spectra.
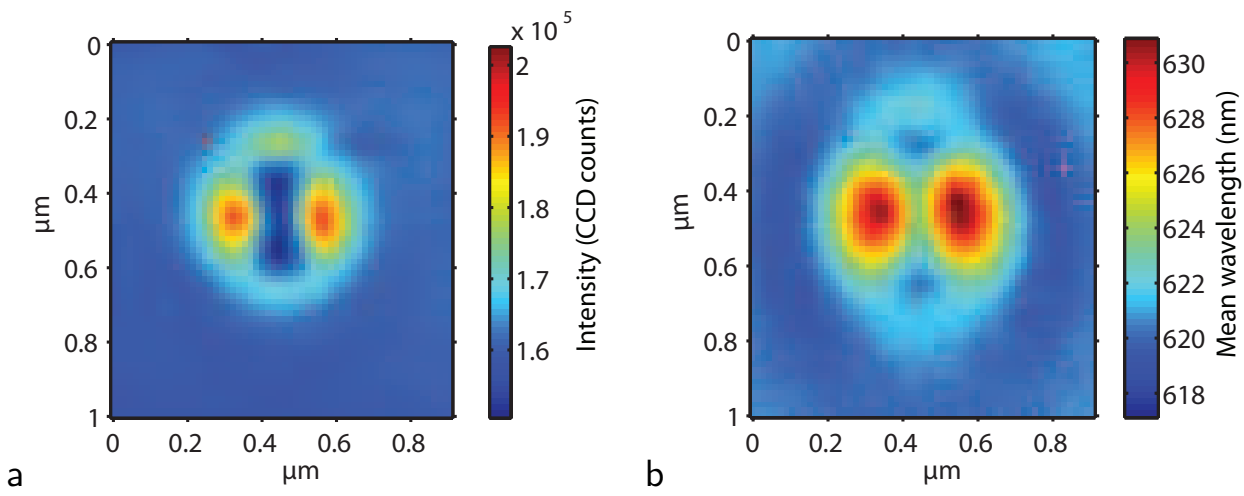

Figure 7.2: (a) Intensity map of a rectangular hole of $100 \times 260 \mathrm{~nm}$. The total intensity collected by the spectrometer is plotted, the graph contains no spectral information. Maxima are visible along the long and short sides of the hole.(b) Map of the average wavelength collected on a rectangular hole of $100 \times 260 \mathrm{~nm}$. This map is constructed from the measured data by calculating the weighted average of the spectrum at each position. This gives good qualitative insight into the observed spectra. The spectra along the long side are clearly red shifted with respect to the spectra collected further from the hole.

In the measurements the most conspicuous features are the two large maxima at the long side of the hole (see Figure 7.2a). At these positions 

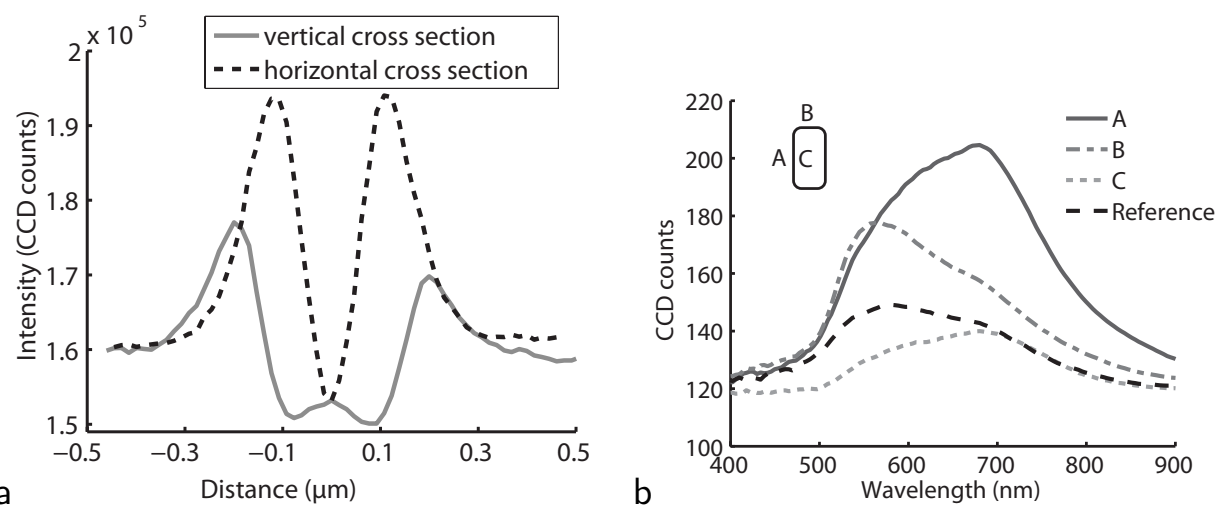

Figure 7.3: (a) Cross sections of figure 7.2. Intensity of transition radiation along two lines through the center of the rectangular hole. Cross section along the short axis: dashed line. Cross section along the long axis: continuous line. (b) Four spectra acquired at different positions on a rectangular hole. A) Spectrum at position $A$ at the peak in intensity at the long side of the hole. B) Spectrum at the peak in intensity at the top side of the hole. C) Spectrum at the center of the hole. Reference) Spectrum corresponds to the background transition radiation obtained at the far corners of the image. 


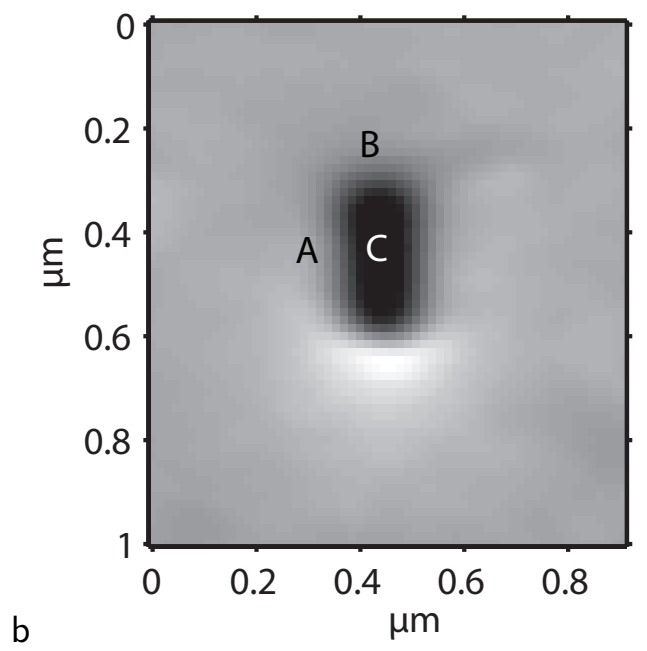

Figure 7.4: Scanning electron microscopy image acquired simultaneously with the intensity map of figure 7.2. With the letters $A, B$ and $C$ positions are indicated where the spectra are as shown in figure 7.36 .

the signal collected is $25 \%$ higher than the signal collected on a plain gold surface. The spatial extent of these maxima is very small; the full width half maximum of these peaks is $120 \mathrm{~nm}$ (see Figure 7.3a). Apart from the two maxima along the long side, also two smaller maxima along the short side of the hole are visible in the intensity map. These two maxima have a difference in intensity that based on the symmetry of the hole is not expected. We attribute this small asymmetry to an angle dependent collection efficiency of the parabolic mirror along one axis.

The spectra at the positions of the intensity maxima of the long side (position A) and top side (position B) are shown in Figure 7.3b together with a spectrum collected on a plain gold surface. Clearly there is a large difference in the spectra at these different positions. The spectrum along the long edge has a peak around $680 \mathrm{~nm}$ while the spectrum at the top side peaks at $560 \mathrm{~nm}$. All spectra measured have only a single maximum. In Figure $7.2 \mathrm{~b}$ it can be seen that indeed around the long edges a red shift with respect to the spectrum far from the hole is visible. At the top and bottom peaks along the short side of the hole the spectrum is blue shifted.

The results discussed for this particular hole are very general for all 
small $(<500 \mathrm{~nm})$ rectangular holes. All holes show single peaked maxima that are more intense as the side of the rectangle is longer. At the sides of holes larger than $500 \mathrm{~nm}$, the maximum along the side of the hole shows multi modal behavior; for a single wavelength multiple peaks can be observed in the spatial map. The results of these measurements are not presented in this thesis. The high peaks at the side of the hole are reminiscent of the high fields that are found on the sides of the hole in theoretical calculations [42]. Since the spectra at the long and short side of a rectangular hole differ considerably, it is tempting to link these peaks and their spectra with the two orthogonal waveguide modes of a rectangular hole.

\subsection{Systematic investigation of the effect of hole shape}

To perform a systematic study of the role of hole shape a sample was made in which the width and height of holes was varied between 50 and $600 \mathrm{~nm}$. At more than 100 holes with different dimensions a spectrum was collected at the region besides the hole as indicated schematically in Figure 7.6.

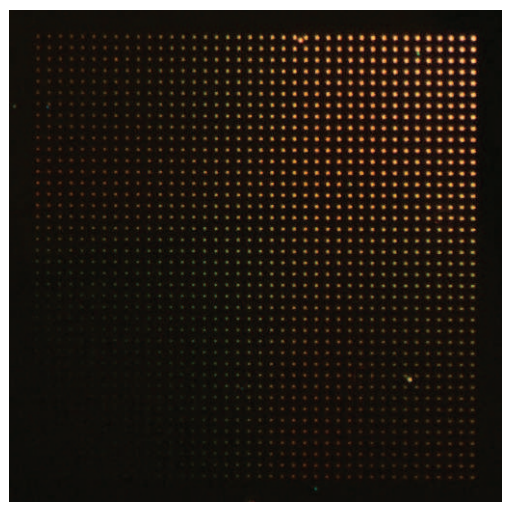

Figure 7.5: Dark field image of a sample in which the hole size is systematically varied. Along the horizontal axis the width of the holes is varied between 50 and $600 \mathrm{~nm}$, while along the vertical axis the height is varied over the same scale. The center to center hole separation is $4 \mu \mathrm{m}$.

The results of these measurements are shown in Figures 7.6, 7.7, 7.8 
and 7.9. As before, all the observed spectra are single peaked. We use the mean wavelength measured in each spectrum as a qualitative measure of the observed spectrum. This quantity gives a good qualitative view on the measured spectra. Figure 7.6 is a color scale plot of the entire data set. It shows the mean wavelength as a function of hole width and height. Cross sections through this data set are shown in Figures 7.7 and 7.8. These two figures show the mean wavelength as a function of the hole width and height. Each figure contains multiple graphs for holes of respectively different height and different width. Figure 7.7 shows the mean spectrum

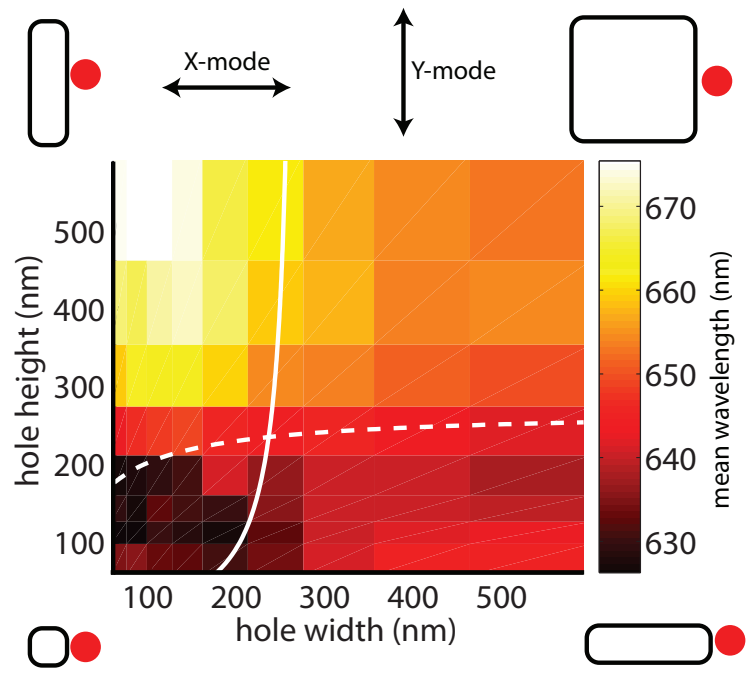

Figure 7.6: measured mean wavelength as a function of the hole dimensions. The schematic figures of the hole illustrate how the size of the hole varies throughout the figure, the red dot indicates the position where was measured. The two white lines indicate the cutoff for $650 \mathrm{~nm}$ for the $X$ mode (horizontal cutoff line) and Y-mode (vertical cutoff line).

at the right side of the hole as the width of the hole is changed. Clearly in the range 300-600 $\mathrm{nm}$ the width of the hole has very little influence on the spectrum collected. Below a width of $300 \mathrm{~nm}$ all the curves have a basic different behavior depending on their height. Below $210 \mathrm{~nm}$ height the mean spectrum decreases as the width is reduced. Above $210 \mathrm{~nm}$ height, the mean spectrum first increases as the width is decreased in the region 
$300-100 \mathrm{~nm}$. Finally in the region below $100 \mathrm{~nm}$ width a small decrease is visible for the 3 spectra with largest heights.

Looking at Figure 7.8 we see the mean spectrum detected as we excite at the right side and vary the length of this side. Each curve corresponds to a series of holes with a constant width. For the holes with widths 60 and $125 \mathrm{~nm}$ the mean spectrum decreases steeply for heights below $300 \mathrm{~nm}$. For holes with larger heights this trend is much less clear.

Furthermore the effect of the hole dimensions on the intensity of the signal can be observed in Figure 7.9. In the figure the averaged intensity over a region around the right side of the aperture is plotted versus the height of this top side. Again the figure contains multiple graphs for different hole widths. The results show that as the hole height increases, the intensity increases until it saturates at a width of $300 \mathrm{~nm}$. This behavior is similar for holes with different width.

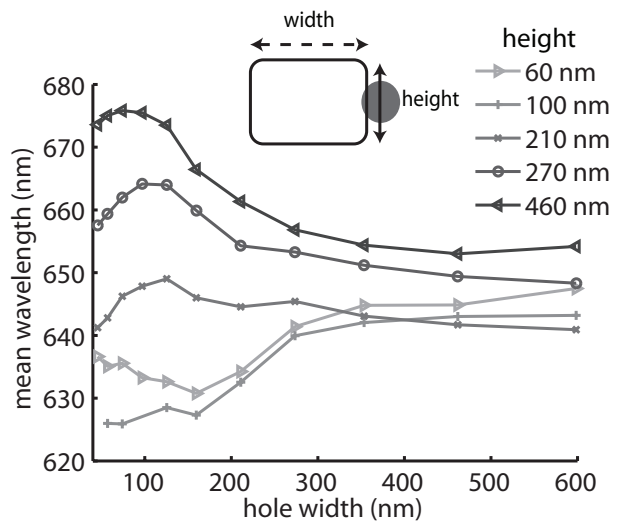

Figure 7.7: Mean wavelength of the spectrum collected at the right side of the hole as a function of the hole width, for different hole heights. In the five curves the hole height is $60 \mathrm{~nm}(\triangleright), 100 \mathrm{~nm}(+), 210 \mathrm{~nm}(\times), 270$ $\mathrm{nm}(\mathrm{O})$ and $460 \mathrm{~nm}(\triangleleft)$ respectively. Above a width of $300 \mathrm{~nm}$ the mean spectrum shows little dependence on the width of the hole. Below $300 \mathrm{~nm}$ the width of the hole has a large influence on the mean spectrum: below a hole height of $200 \mathrm{~nm}$ there is a decrease in mean wavelength while for holes with larger height a decrease in width leads to a red shift of the mean spectrum. 


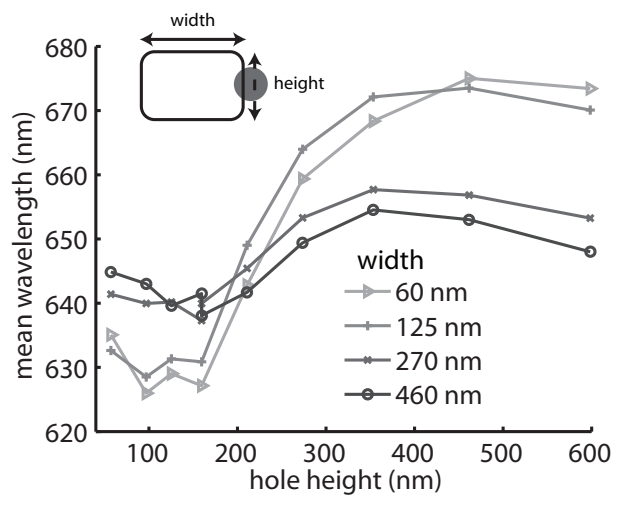

Figure 7.8: Mean wavelength of the spectrum collected at the right side of the hole as a function of the hole height, for different hole widths. In the four curves the hole width is $60 \mathrm{~nm}(\triangleright), 125 \mathrm{~nm}(+), 270 \mathrm{~nm}(\times), 460$ nm (०) respectively. 


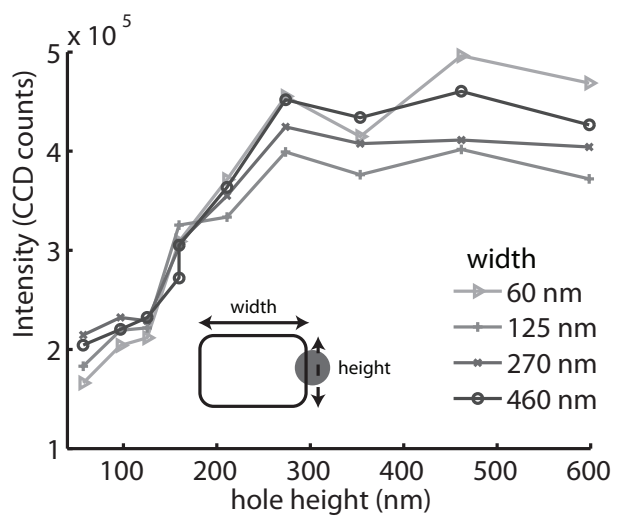

Figure 7.9: Measurement of the intensity of the transition radiation at the right maximum of a rectangular hole as a function of the hole width, for different hole heights. In the four curves the hole width is $60 \mathrm{~nm}(\triangleright), 125$ $\mathrm{nm}(+), 270 \mathrm{~nm}(\times), 460 \mathrm{~nm}(\mathrm{O})$ respectively. The intensity is a dependent on the height, especially in the range below $300 \mathrm{~nm}$. The intensity is less dependent on the hole width.

\subsection{Interpretation}

In Chapter 4 we found that the spectrum of the surface wave and the direct transition radiation are not identical. Figure 7.10 shows the normalized spectrum of the transition radiation and the surface wave at two different distances from the impact site of the electron. We observe that the spectrum of the transition radiation has a mean wavelength that is to the red of the spectra of the surface waves. Thus one would naively expect the spectrum measured near a hole to become blue shifted if the hole scatters the surface. This is opposite to what was observed in Figure $7.2 \mathrm{~b}$ where the spectra near the hole have a mean wavelength that is $630 \mathrm{~nm}$ while the spectra further away have a mean wavelength of $620 \mathrm{~nm}$. We conclude from this that the shape of the holes must have an important influence on the measured spectra.

The ability of subwavelength holes to scatter light to SPPs was discussed in Section 2.6.1. Experiments by Baudrion and co-workers [41] showed that the scattering of light to SPPs is considerably more efficient for larger holes than for smaller holes. Moreover the highest efficiency for SPP launching by 


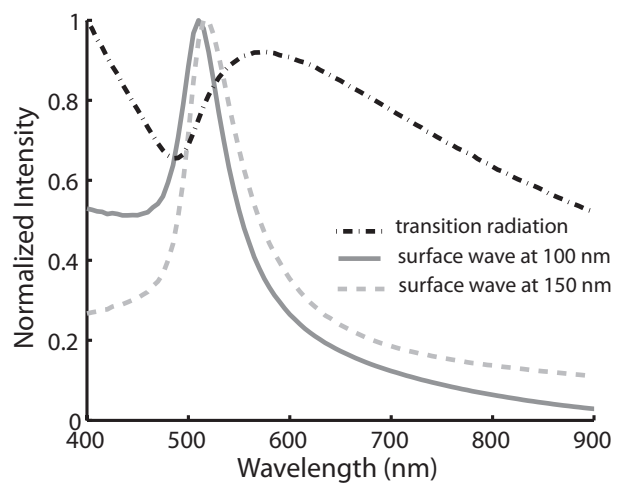

Figure 7.10: Transition radiation spectra at 3 different positions, normalized to the peak value. Transition radiation spectrum, spectrum at the surface $100 \mathrm{~nm}$ from the electron impact site, spectrum at the surface $150 \mathrm{~nm}$ from the electron impact site. The detection efficiency is not taken into account in these spectra.

the hole was observed at the cutoff wavelength of the hole. In an analogous way the scattering of the field of the electron at the interface will depend critically on the hole and the modes of the hole. A very small hole, for which al wavelengths are above the cutoff wavelength will show less scattering than a hole that has propagating waveguide modes. In Figure 7.6 the mean wavelength as a function of hole size is plotted. The two curves indicate the hole dimensions with a cutoff at $650 \mathrm{~nm}$ using the model that was introduced in Section 2.6.1.

With the discussed behavior of the cutoff in mind we propose the following explanation of the observed dependence of the mean spectrum on the dimensions of the hole. The transition radiation generated close to the hole increases as there is a waveguide mode in the hole available. This might seem counter intuitive as one might naively think that if there is a waveguide mode light can only disappear. However, the fact that there is a mode also means that the scattering of the hole increases. This increased scattering leads to a larger intensity in the measurement. The spectrum measured close the hole, is thus influenced by the waveguide modes of the hole.

We now consider Figure 7.6. For the holes on the top right side, most 
of the wavelengths are above cutoff, decreasing their width, moving to the top right side of the graph will lead to a red shift of the cutoff wavelength, leading to a larger contribution of the long wavelengths to the spectrum scattered from the hole. Starting in the lower right side, we are already below cutoff for all wavelengths. Decreasing the width of the hole we observe a blue shift of the spectrum. Here the reasoning is that a non-resonant scatterer will always scatter short wavelengths more efficiently than long wavelengths. Therefore decreasing the size of holes that are already in cutoff will lead to a blue shift in the observed spectrum.

The qualitative reasoning above simplified the explanation to low scattering below cutoff and high scattering above cutoff. These statements can be refined further by realizing that the scattering depends on the specific propagation properties of the waveguide and not solely on it being above or below cutoff. Our approach is a useful tool to understand transition radiation microscopy measurements near small plasmonic structures. 


\section{Chapter 8}

\section{Polarization-resolved scattering from subwavelength holes}

The polarization of the transition radiation generated close to a small rectangular hole is analyzed. Under the assumption that the light generated, is mostly scattered perpendicular to the surface, the results show that the polarization of the light that is generated is mainly perpendicular to the edge at which the electron beam is placed. This is in agreement with the formulated expectations.

\subsection{Introduction}

The optical properties of gold are such that polarization effects play an important role in the interaction of light with plasmonic nano-structures made of this noble metal. Knowledge of the polarization of light scattered by rectangular subwavelength holes is therefore of great value to further the understanding of the interaction of light with these structures. Far-field methods have been used to obtain the polarization dependent transmission through rectangular holes [2] and the coupling efficiency of the impinging light to surface plasmon polaritons [41]. Also polarization resolved nearfield scanning optical microscopy on single holes has been performed [84]. Both far-field and near-field methods are however unable to resolve how hole shape influences this behaviour. With transition radiation microscopy, 
polarization dependent information with a much higher resolution can be obtained, as was shown by Degiron and co-workers [2] for circular holes. In this chapter we will discuss polarization resolved measurements on a rectangular hole.

\subsection{Induced dipole moments in a rectangular hole}

A helpful concept that is often used in the description of polarization effects in metal holes is the induced surface charge distribution in the quasi-static limit. The quasi-static limit is valid when the structure is much smaller than the wavelength of the light, which is a reasonable assumption for the rectangular hole with dimensions $100 \times 260 \mathrm{~nm}$ that we study. The surface charge distribution induced by the illumination of a hole with linearly polarized light is depicted schematically in Figure $8.1[85,86]$. The corresponding induced dipole moment is oriented parallel to the inducing field. If we excite the structure at an edge it is impossible to induce a dipole moment along the edge due to the good conductivity of the metal. Across the hole a dipole moment can be induced however and this means that the preferential polarization of the field radiated by the induced dipole is perpendicular to the edge.

An alternative way to argument what the polarization scattered from the hole will be -giving the same result- is the efficiency to launch surface plasmon polaritons at a metal edge. To efficiently launch surface plasmon polaritons at an extended metal edge, the polarization of the light has to be chosen perpendicular to the edge. The reason for this is that the symmetry of the applied field has to match the symmetry of the electric field of the plasmon. For light polarized parallel to the edge this is not the case. In the experiments described in this thesis, the inverse process is studied: by means of the electron beam, surface waves are generated that scatter from the hole to the far-field. Based on reciprocity this will lead to scattered light with a specific polarization.

\subsection{Polarization selection}

The setup (see Chapter 5, Figure 5.2) has the possibility to introduce a polarizer in the beam path. This way the contributions of the different 


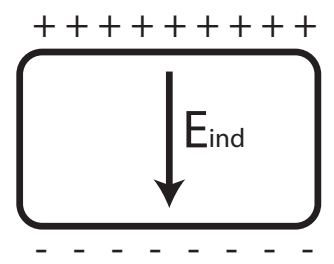

Figure 8.1: Surface charge distribution induced by light impinging on a hole with its polarization oriented along the arrow.

polarizations can be analyzed. The polarization dependent collection properties of the parabolic mirror are however not trivial. In a simple example we will illustrate that one has to be careful while interpreting polarization resolved measurements. We consider a dipole source $\alpha$ in the focal point of a parabolic mirror, with its dipole moment oriented along the axis of the paraboloid (see Figure 8.2). Following the rays from the dipole perpendicular to its dipole moment, the reflected rays form a collimated beam emerging from the mirror. This beam will be radially polarized. Measuring the intensity of the beam depending on the angle of the analyzer will give no clear information on the orientation of the dipole. From this we could conclude that the polarization of the light emitted by the dipole is lost. However, when the light is scattered from the structure in a preferential direction it is very well possible that information on the polarization is still present. Additionally when unpolarized light impinges on the aluminium parabolic mirror the reflected light will not become polarized. This suggests that if polarization dependencies are observed they have to be related with a polarization dependent emission.

Based on the previous discussion it is difficult to argue what the relation between the polarization of the emitted light and the polarization of the collected light is. We will assume here that the light scattered at the subwavelength hole is predominantly directed straight up from the surface. Then the polarization emitted and collected are related to each other as indicated by the $\mathrm{X}$ and $\mathrm{Y}$ polarization drawn in the schematic illustration of Figure 8.2. 

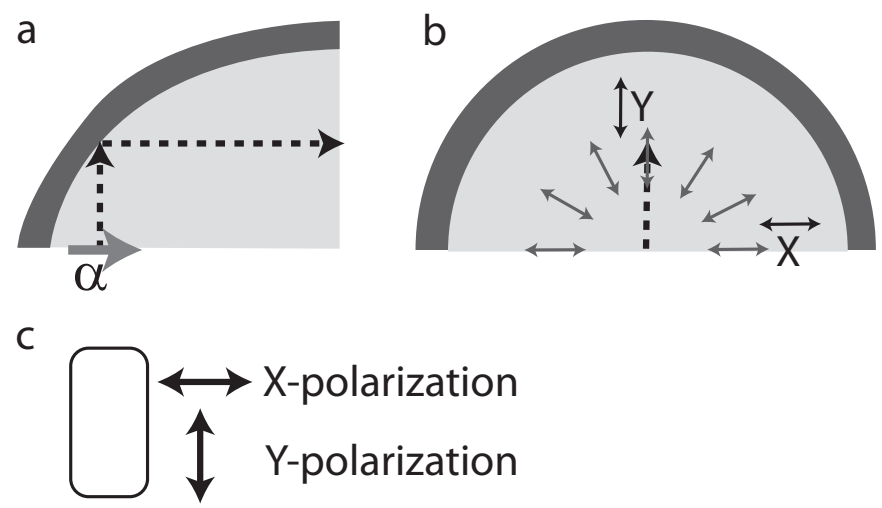

Figure 8.2: Two cross sections of the parabolic mirror (figure a and b). The dashed arrows indicate a light path, the thin arrows indicate polarization directions. A dipole $\alpha$ oriented parallel to the surface, with its dipole moment pointing along the axis of the paraboloid, will emit light that is radially polarized after the mirror. In the interpretation of the measurements presented in this chapter, it is assumed that most of the collected light is scattered by the hole straight up from the surface. This means that the $X$ and $Y$-polarization emitted from the sample indicated schematically by the rectangular hole (figure $c$ ) are mapped on the $X$ and $Y$-polarization as indicated in the cross section on the right side (figure $b$ ). 


\subsection{Results}

Figure 8.3 shows the result of 16 intensity maps measured of the same hole, rotating the analyzer after the parabolic mirror in steps of 22.5 degrees over 360 degrees. Clearly the light collected by the system has polarization dependent properties as the maps differ distinctively for different orientations of the polarizer. The collected light consists of a not-linearly polarized part and a linearly polarized part. Generally the not-linearly polarized part consists of an unpolarized part, a circular polarized part and a part that arises from the spatial variation of the linear polarization within the beam. The current measurements can not be used to distinguish these contributions.

Per pixel position the function $S_{\theta}=I_{u}+I_{p} * \cos ^{2}\left(\theta-\theta_{p}\right)$ is fitted to the measurements, where $S_{\theta}$ is the intensity measured on each pixel for different orientation angles $\theta$ of the analyzer. $I_{u}, I_{p}$ and $\theta_{p}$ are fit parameters that correspond to the intensity of the not-linearly polarized part, the intensity of the linearly polarized part and the orientation of the linearly polarized part respectively. Figure 8.4 shows the obtained values of $I_{u}$ and $I_{p}$ in identical color scales. Note that the polarized intensity $I_{p}$ is larger than the unpolarized. In Figure 8.5 the intensity $I_{p}$ and the angle $\theta_{p}$ are plotted as headless arrows. The polarization of the emission is oriented perpendicularly to the edges of the hole on the right, left and lower part of the hole. In the upper part of the hole the polarization is oriented parallel to the edge of the hole, but here the linearly polarized contribution is lower in amplitude. The asymmetry that is observed on the top and bottom side is most probably related to the asymmetric collection efficiency of the parabolic mirror.

\subsection{Conclusion and discussion}

Although one must be careful in interpreting the results of polarizationdependent measurements obtained with a parabolic mirror, the choice made in relating the angle of the polarizer to the polarization of the emitted light seems to be correct, as the results presented in Figure 8.4 are in good agreement with the expectations formulated in Section 8.2. The scattered field is polarized perpendicular to the edge from which it is scattered. This is also in correspondence with an induced surface charge distribution as schematically depicted in Figure 8.1. 

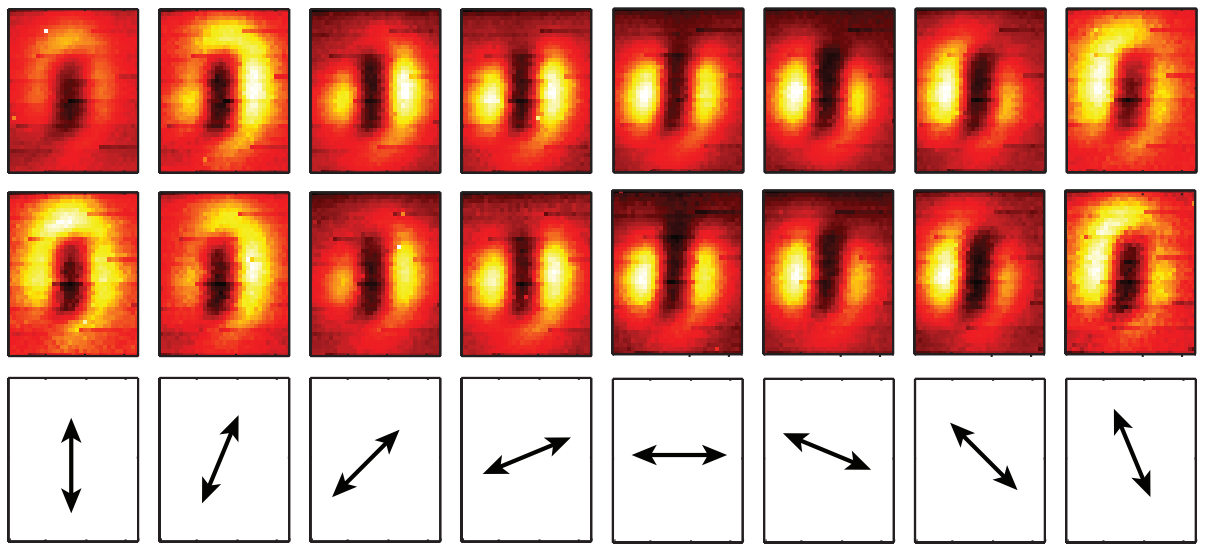

Figure 8.3: 16 intensity maps of the same rectangular hole for 16 settings of the analyzer, rotating the polarizer over $2 \pi$ radiants.

a
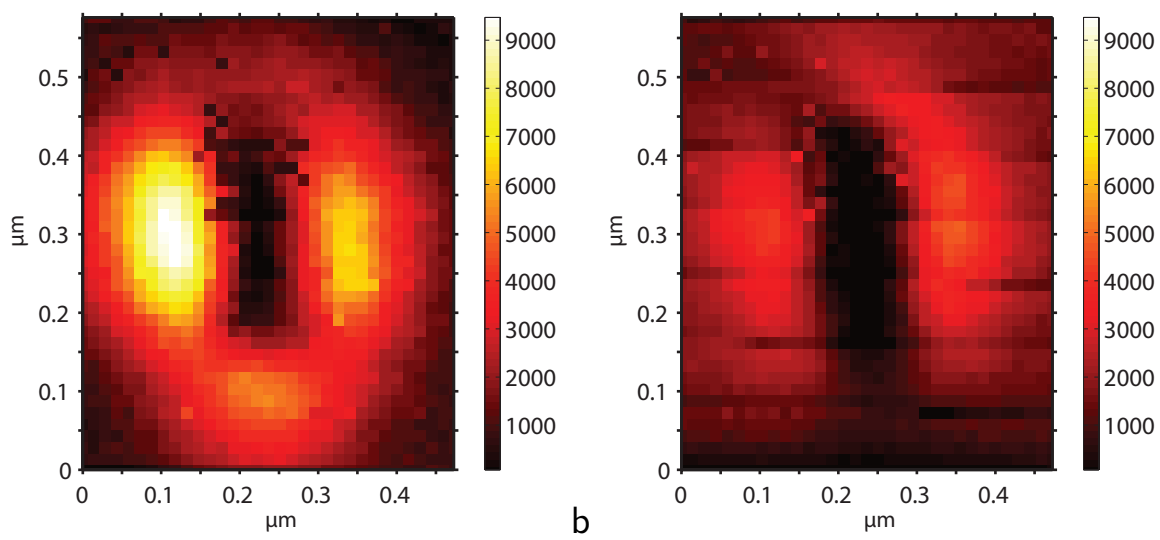

Figure 8.4: Polarization distribution reconstructed from 16 measurements of a single hole. a) The intensity map of $I_{p}$, the amplitude of the linearly polarized contribution. b) The intensity map of $I_{u}$, the amplitude of the unpolarized contribution. 


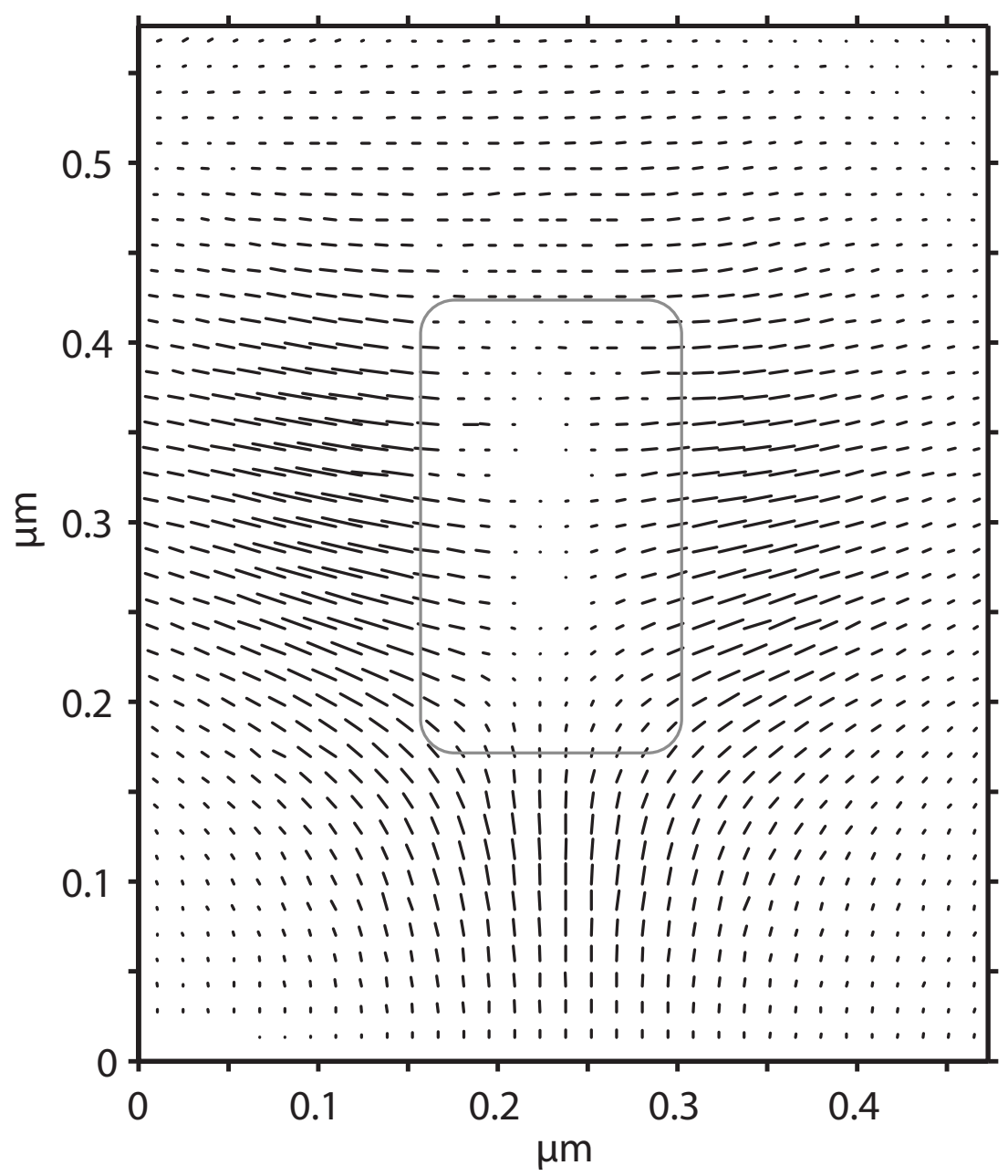

Figure 8.5: Polarization distribution reconstructed from 16 measurements of a single hole. At every position a headless arrow indicates the magnitude and orientation of the linearly polarized part $\left(I_{p}\right)$. 


\section{Chapter 9}

\section{Scattering from multiple holes}

Transition radiation microscopy measurements on structures consisting of three subwavelength holes are discussed. A large influence of hole spacing is found. The collected spectra are shifted by $75 \mathrm{~nm}$ by changing the spacing between the holes from 240 to $350 \mathrm{~nm}$. This shows that no long range periodicity is necessary to achieve considerable shifts in the spectral response of these structures.

\subsection{Introduction}

When an array of subwavelength holes is illuminated by a plane wave each hole acts as a scatterer at which surface waves are generated. These surface waves can subsequently scatter or reflect at the other holes present in the structure. This scattering process leads to the reflection and transmission of light from the complete structure. In this chapter we try to decompose this complicated problem by looking at only 3 holes. Similar structures have been measured using far-field methods where the entire array is illuminated [87]. In such experiments all the holes are driven in unison. With transition radiation microscopy we can locally excite this relatively simple geometry. In the experiment we will vary the distance between these holes and investigate the spectra measured at several different positions. 


\subsection{Results}

In Figure 9.1 transition radiation microscopy intensity images of three configurations of three subwavelength holes are shown. In each image the holes have a width of 260 and a height of $100 \mathrm{~nm}$. The distance D between the holes is different for each triplet.
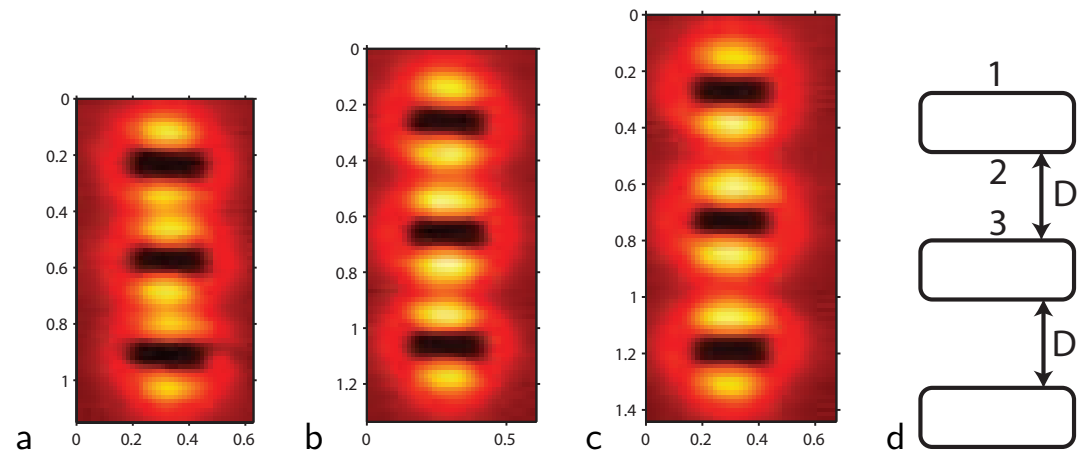

Figure 9.1: Intensity maps of 3 configurations of rectangular holes and a schematic overview. The figures $a, b$ and $c$ all have holes of the size 100 $\times 260 \mathrm{~nm}$. The distance between the holes is 240 (a), 300 (b) and 350 $n m$ (c). In the schematic overview (d) the positions 1, 2 and 3 indicate the positions where spectra are shown in figure 9.2. All three pictures have identical scaling, the axes are in $\mu \mathrm{m}$.

Figure 9.2a shows the spectra acquired when the electron beam is positioned at the top side of the upper hole of the triplet (position 1 in the schematic overview in Figure 9.1d). The graphs a, c and e are for the three triplets with different spacing. This same data is shown in graphs $b, d$ and $f$ but here the spectra are normalized: the plotted value is $N(\lambda)=I_{\text {det }}(\lambda) / I_{\text {ref }}(\lambda)$ with $I_{\text {det }}(\lambda)$ the detected spectrum and $I_{r e f}(\lambda)$ a reference spectrum obtained at the long side of a single isolated hole of the same shape. The graphs in both figures show the spectra and normalized spectra at respectively position 1 (a,b), 2 (c,d) and 3 (e,f) (Figure 9.1d). The positions 1, 2 and 3 are all located in the high intensity peaks just above or below the hole.

The first observation to be made is that all normalized spectra have values mainly above 1 . The intensity of the spectra increases going from 


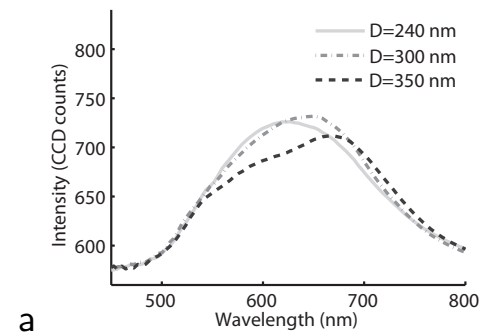

a

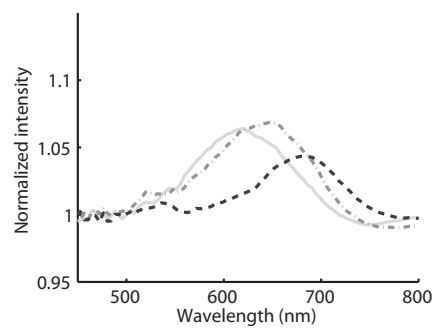

C
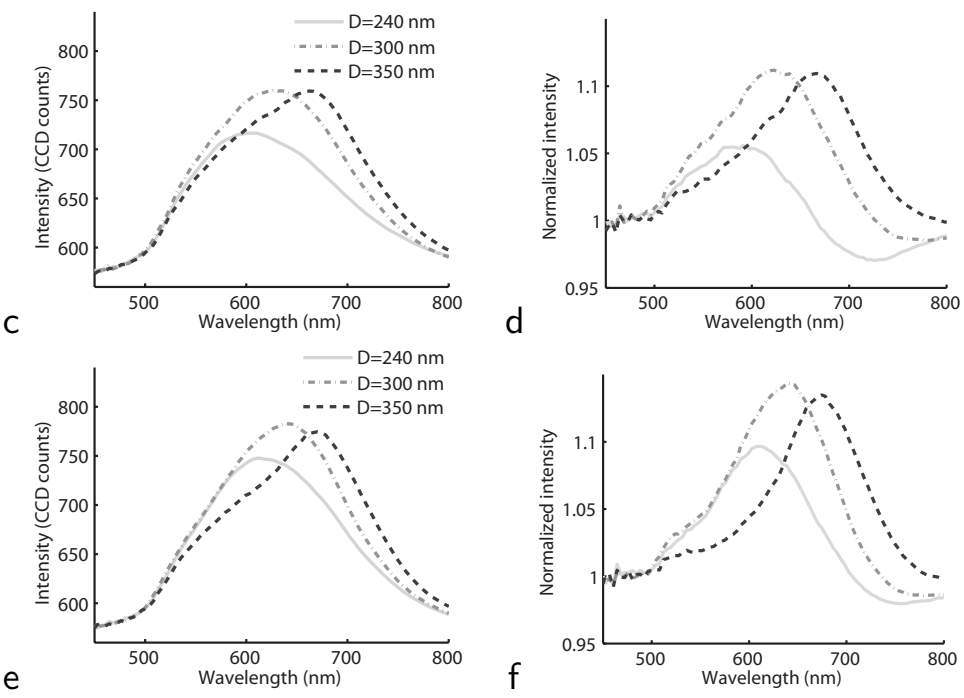

Figure 9.2: Spectra at 3 positions for each position varying the hole spacing (a,c,e) and normalized spectra at 3 positions for each position varying the hole spacing $(b, d, f)$. (a) The spectra at position 1 at the top side of the upper hole. (c) The spectra at the position 2 at the bottom side of the top hole. (e) The spectra at the top side of the top side of the middle hole. (b) The spectra at position 1 at the top side of the upper hole. (d) The spectra at the position 2 at the bottom side of the top hole. (f) The spectra at the top side of the top side of the middle hole. The plotted value is $N(\lambda)=I_{\text {det }}(\lambda) / I_{\text {ref }}(\lambda)$ with $I_{\text {det }}(\lambda)$ the detected spectrum and $I_{r e f}(\lambda)$ the spectrum collected at the top maximum of a isolated hole of the same size and shape. All spectra were obtained in the maxima visible in Figure 9.1, just above or below the hole. 
position 1 to position 2 to position 3 (figures a,b,c). The amount of light radiated from the structure within the measured range of wavelengths, is thus larger than for a single isolated hole. This statement needs some refinement, as it is not true for all wavelengths. The geometry with the smallest spacing gives rise to a small decrease in the spectrum with respect to the single hole, for a small band of wavelengths. Especially at position 2 (Figure 9.2b) a decrease in the wavelengths around $700 \mathrm{~nm}$ can be observed. Another trend visible in the data, is that the peak in both the spectrum and the normalized spectrum shifts to the red when the spacing of the holes is increased. The positions of the peaks in the spectra as a function of the hole spacing are plotted in Figure 9.3.

The observed intensity at every position is generally higher than the intensity measured at a single isolated hole. This behavior of the intensity is in good correspondence with what one intuitively might expect: more holes leads to more scattering of surface waves and thus to a higher observed intensity. At position 2 and 3 from a geometric point of view, more scattering is expected than from position 1 , as the latter position neighbors a large empty field of gold. Thus, the closer to the center of the triplet the more scattering will occur.

The fact that the radiated spectrum changes as the spacing between the holes is varied, is an indication that there is an interference effect leading to the response of the entire structure. Surprisingly, no long range periodicity is necessary to achieve a considerable $(75 \mathrm{~nm})$ shift in the peak position. This is in good agreement with the observation of clear peaks in the transmission spectrum of quasi-periodic structures [88]. Here as well, short range order plays an important role as these structures lack long range periodicity.

We note here that the system under investigation has similarities with an antenna array, as was pointed out by Alaverdyan et al. [87]. The holes act as scatters with a well defined phase difference, given by their distance. In our experiment we can preferentially excite one of the elements of the antenna array. Yet the observed spectrum is the response of the entire structure rather than the response of a single hole. 


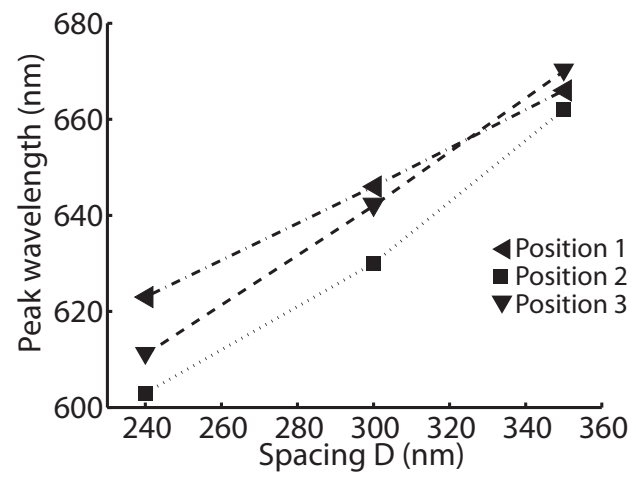

Figure 9.3: Positions of the peaks as a function of hole spacing D for position 1 (४, top side of the top hole), position $2(\mathbf{\square}$, lower side of the top hole) and position 3 ( $\mathbf{\nabla}$, upper side of the middle hole)

\subsection{Conclusions}

Scattering plays an important role in structures consisting of just three holes. The different configurations gives rise to large $(75 \mathrm{~nm})$ shifts of the peaks in the observed spectra. The role of spacing is very important in these structures, the experiments suggest however that long range periodicity is not necessary to change the spectral behavior of structures consisting of subwavelength holes. 

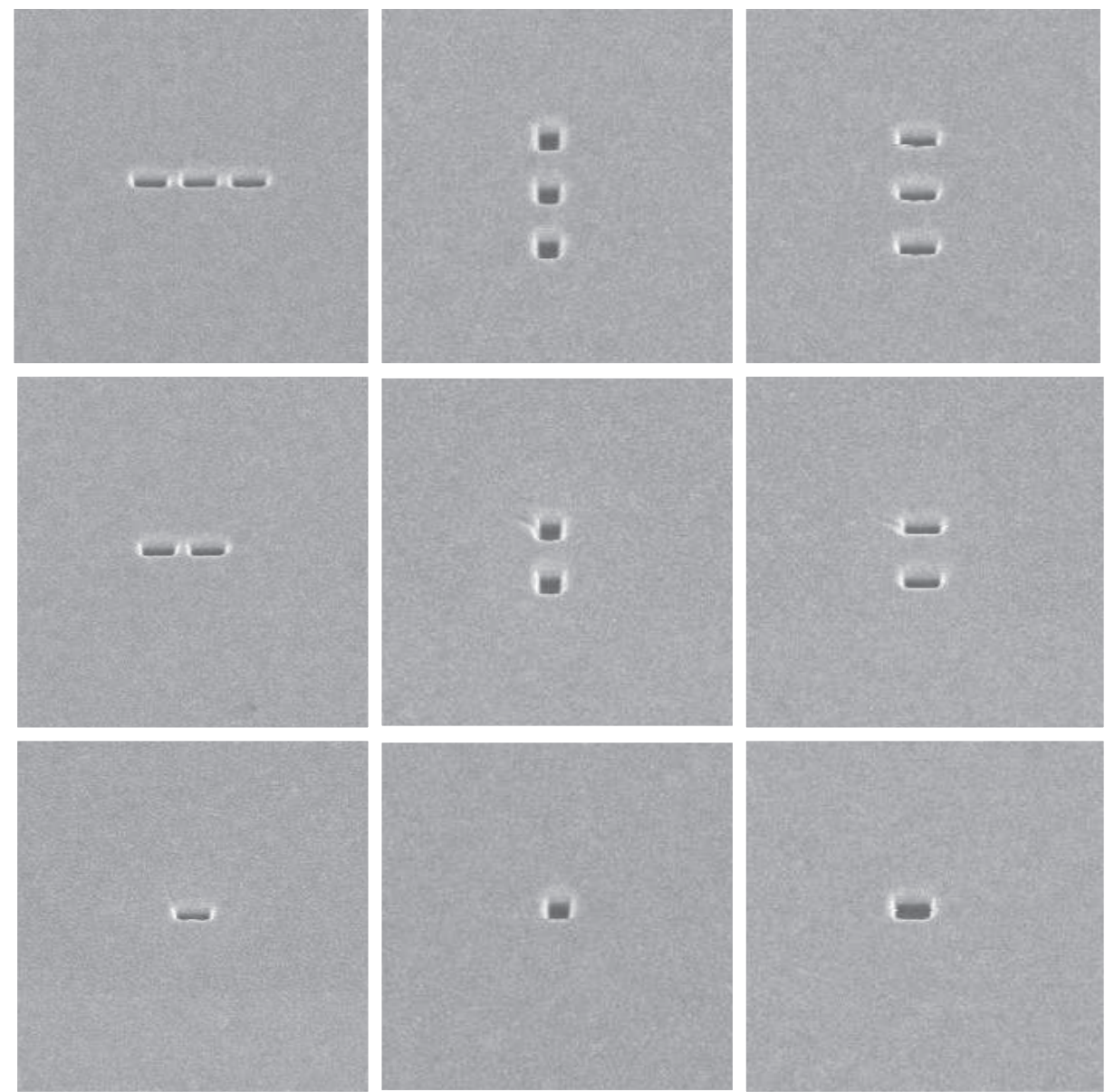

Figure 9.4: Several SEM pictures of holes, taken under 52 degrees. 


\section{Appendix A}

\section{Group delay through attenuating media}

The time delay a pulse experiences when it propagates through a subwavelength hole array yields insight into the dynamic response of the structure. One specific interest in the research of time dynamics in photonic nanostructures is the increase of nonlinear effects when a light pulse has a low velocity [89]. Effects such as second harmonic generation are expected to be enhanced when a pulse resides longer in a structure. One has to be careful however in interpreting the delay of pulses propagating though attenuating media. In such media the common definition of group velocity $v_{g}=\frac{\mathrm{d} \omega}{\mathrm{d} k}[90]$ is not equivalent to the velocity of the envelope of the pulse. This stems from the fact that a combination of group velocity dispersion and attenuation can also lead to a delay of the envelope of a pulse as well. This delay is however related with the reshaping of the pulse and not with a longer presence of the field in the medium. It will therefore cause no enhancement of light matter interactions as slow light does.

In the measurements performed in this thesis the delay of the envelope of the pulse through a subwavelength hole array is determined. The transmission amplitude of these structures has a large wavelength dependence. In this appendix the role of this wavelength-dependent transmission on the propagation of the envelope is investigated. The aim is to formulate the theoretical background for the description and measurement of femtosecond (fs) pulse propagation through media in which the transmission varies as a function of frequency. We will see that an interferometric measurement 
technique has properties that make it a very practical technique to measure group delay. Additionally, we will derive the lowest order term that describes the deviation of the velocity of the envelope from $\frac{\mathrm{d} \omega}{\mathrm{d} k}$. This term will alow us to determine the effect of attenuation on the measurement of group delay.

\section{A.1 Group velocity in absorbing media}

The field of a pulse, propagating along the $x$-direction through an isotropic absorbing medium, can be written as an integral over many harmonic waveforms using a Fourier expansion,

$$
E(x, t)=\int_{-\infty}^{\infty} A(\omega) \mathrm{e}^{\mathrm{i}(k(\omega) x-\omega t)} \mathrm{d} \omega,
$$

where $A(\omega)$ is the complex spectrum of the field. The propagation of the wave in a medium is described by the factor $\exp i k(\omega) x$. Note that $k(\omega)$ is dependent on the frequency $\omega$. In general $k$ is a complex quantity in which the real part $k^{\prime}$ describes the phase evolution while the imaginary part $k^{\prime \prime}$ describes the attenuation in the material. Throughout this chapter this notation using ' for the real part and " for the imaginary part of a complex quantity will be used. We will consider here the situation in which $A(\omega)$ is sufficiently narrow in bandwidth such that we can approximate the wavevector $k$ with a Taylor series around $\omega=\omega_{0}$.

$$
k(\omega)=\beta_{0}^{\prime}+\beta_{1}^{\prime}\left(\omega-\omega_{0}\right)+\mathrm{i} \beta_{0}^{\prime \prime}+\mathrm{i} \beta_{1}^{\prime \prime}\left(\omega-\omega_{0}\right)+\ldots
$$

To simplify the notation, different orders of derivation are written as $\beta_{n}=$ $\left.\frac{\mathrm{d}^{n} k}{\mathrm{~d} \omega^{n}}\right|_{\omega 0}$. In this section we will firstly limit ourselves to the first two terms. The expression for $k(\omega)$ can be substituted in Equation A.1. This yields

$$
E(x, t)=\int_{-\infty}^{\infty} \tilde{A}(\omega) \mathrm{e}^{-\beta_{0}^{\prime \prime} x-\beta_{1}^{\prime \prime}\left(\omega-\omega_{0}\right) x} \mathrm{e}^{\beta_{0}^{\prime} x-\omega_{0} t} \mathrm{e}^{\mathrm{i}\left(\beta_{1}^{\prime} x-t\right)\left(\omega-\omega_{0}\right)} d \omega .
$$

Now it is possible to separate the carrier wave from the envelope,

$$
E(x, t)=\mathrm{e}^{\mathrm{i}\left(\beta_{0}^{\prime} x-\omega_{0} t\right)} \mathrm{e}^{-\beta_{0}^{\prime \prime} x} \int_{-\infty}^{\infty} \tilde{A}(\omega) \mathrm{e}^{-\beta_{1}^{\prime \prime}\left(\omega-\omega_{0}\right) x} \mathrm{e}^{\mathrm{i}\left(\beta_{1}^{\prime} x-t\right)\left(\omega-\omega_{0}\right)} d \omega .
$$

The first term outside the integral describes a wave with the phase velocity of the carrier wavelength. The second term outside the integration, containing the term $\beta_{0}^{\prime \prime}$, describes the attenuation in the medium. This term 
has no influence on the pulse delay but only on the transmitted amplitude. The complex exponent inside the integral describes the propagation of the envelope where the group velocity is $v_{g}=\frac{1}{\beta_{1}^{\prime}}=\frac{\mathrm{d} \omega}{\mathrm{d} k^{\prime}}$. Though we are not taking into account group velocity dispersion, the frequency-dependent losses given by $\beta_{1}^{\prime \prime}$, might cause deformation of the initial envelope depending on the exact form of $A(\omega)$.

\section{A.1.1 The Fourier-limited pulse}

The effect of the attenuation on the propagation of Fourier-limited and chirped pulses with a Gaussian spectrum can be described analytically. We first consider a Fourier-limited pulse. The expression for the spectral amplitude is then

$$
A(\omega)=\mathrm{e}^{-\frac{\left(\omega-\omega_{0}\right)^{2}}{\Delta \omega^{2}}},
$$

where $\Delta \omega$ describes the spectral width of the pulse. The integration in Equation A.1 can be performed changing the integration variable $\omega^{\prime}=$ $\omega-\omega_{0}$,

$$
E(x, t)=\mathrm{e}^{\mathrm{i}\left(\beta_{0}^{\prime} x-\omega_{0} t\right)} \mathrm{e}^{-\beta_{0}^{\prime \prime} x} \int_{-\infty}^{\infty} \mathrm{e}^{-\frac{\omega^{\prime 2}}{\Delta \omega^{2}}} \mathrm{e}^{-\beta_{1}^{\prime \prime} \omega^{\prime} x} \mathrm{e}^{\mathrm{i}\left(\beta_{1}^{\prime} x-t\right) \omega^{\prime}} d \omega^{\prime} .
$$

The expression for the field then yields

$$
E(x, t)=\mathrm{e}^{\mathrm{i}\left(\beta_{0}^{\prime} x-\omega_{0} t\right)} \mathrm{e}^{-\beta_{0}^{\prime \prime} x} \Delta \omega \pi \mathrm{e}^{-\frac{1}{4} \Delta \omega^{2}\left(t-\beta_{1}^{\prime} x\right)^{2}} \mathrm{e}^{\frac{1}{4} \Delta \omega^{2}\left(\beta_{1}^{\prime \prime} x\right)^{2}} \mathrm{e}^{\frac{1}{2} \mathrm{i} \Delta \omega^{2}\left(t-\beta_{1}^{\prime} x\right) \beta_{1}^{\prime \prime} x} .
$$

In which an envelope moving with velocity $1 / \beta_{1}^{\prime}$ can be recognized in the exponent $\exp \left(-\frac{1}{4} \Delta \omega^{2}\left(t-\beta_{1}^{\prime} x\right)^{2}\right)$. The term $\exp \left(\frac{1}{4} \Delta \omega^{2}\left(\beta_{1}^{\prime \prime} x\right)^{2}\right)$ causes a spatial broadening of the pulse. Such a broadening does not influence the velocity of the envelope since it does not displace the center of the pulse. The last exponent is a pure phase factor that will not influence the propagation velocity of the envelope. We conclude from this that the group delay of the Fourier-limited pulse is not influenced by attenuation.

\section{A.1.2 The chirped pulse}

In case of a chirped pulse with a Gaussian spectrum the delay is no longer independent of the attenuation. We consider a chirped pulse,

$$
\tilde{A}(\omega)=\mathrm{e}^{\frac{\left(\omega-\omega_{0}\right)^{2}}{\Delta \omega^{2}(1+\mathrm{i} \gamma)}}
$$


where $\gamma$ is a quadratic phase factor that describes the frequency chirp of the pulse. We then obtain an expression for the field

$$
E(x, t)=\mathrm{e}^{\mathrm{i}\left(\beta_{0}^{\prime} x-\omega_{0} t\right)} \mathrm{e}^{-\beta_{0}^{\prime \prime} x} \pi \Delta \omega \mathrm{e}^{-\frac{1}{4} \Delta \omega^{2}(1+\mathrm{i} \gamma)\left(t-\left(\mathrm{i} \beta_{1}^{\prime \prime}+\beta_{1}^{\prime}\right) x\right)^{2}}
$$

from which we can derive the expression for the envelope

$$
|E(x, t)|=\pi \Delta \omega \mathrm{e}^{-\beta_{0}^{\prime \prime} x} \mathrm{e}^{-\frac{1}{4} \Delta \omega^{2}\left(\left(t-\beta_{1}^{\prime} x\right)^{2}+\left(\beta_{1}^{\prime \prime} x\right)^{2}\right)} \mathrm{e}^{-\frac{1}{4} \Delta \omega^{2} \gamma \beta_{1}^{\prime \prime} x\left(t-\beta_{1}^{\prime} x\right)} .
$$

Now the last term describes a deformation of the Gaussian pulse shape due to the chirp factor $\gamma$. It contains terms of both $t$ and $\beta_{1}^{\prime \prime}$. This means that the attenuation term leads to a deformation of the envelope that shifts the center of the envelope. From this we conclude that the delay of the pulse is dependent on its initial chirp.

\section{A.2 Measurement method for the group delay}

As we have seen, a combination of an absorbing medium and the initial chirp of the input pulse can lead to a change in pulse delay. This suggests that an involved characterization of the pulses used to measure the group delay through a structure is necessary. To avoid such complicated procedure, we chose to use a linear interferometric method. As we will show this method eliminates the effect of the initial chirp of the pulse. Still there is an effect of attenuation on the observed delay in this technique as well. We will derive an equation that will allow us to determine the magnitude of this effect.

\section{A.2.1 Transfer function}

In the previous section we considered pulse propagation through isotropic media. Here, instead of using a detailed picture of how the light propagates through the medium, we will describe the sample and other components by their complex transfer function $T(\omega)$. This formalism considers a component as a 'black box' with a specific frequency response. In this way we will be able to describe the effect of attenuation on the observed group delay as general as possible.

The propagation through a structure leads to a group delay that can be deduced from the complex transfer function. Using

$$
T_{s}(\omega)=\mathrm{e}^{\mathrm{i}\left(k^{\prime}(\omega)+\mathrm{i} k^{\prime \prime}(\omega)\right) L},
$$


where $k(\omega)$ is the wavevector in an effective medium with thickness $L$. The group velocity $\frac{d \omega}{d k}$ can be calculated from $T_{s}(\omega)$

$$
\begin{aligned}
& k^{\prime}(\omega)=\arg \left[T_{s}(\omega)\right] / L, \\
& \frac{d \omega}{d k}=L \frac{d \omega}{d \arg \left[T_{s}(\omega)\right]} .
\end{aligned}
$$

The group delay follows from this

$$
\tau_{g}=L / v_{g}=\frac{d \arg \left[T_{s}(\omega)\right]}{d \omega} .
$$

This last relations gives the group delay, which is not equivalent to the delay of the envelope of the pulse in media with attenuation, as was made clear in the previous section. In the most general case the effect of a medium on the propagation of a pulse is described by a transfer function written in the form

$$
T(\omega)=\mathrm{e}^{\mathrm{i} \alpha(\omega)},
$$

with $\alpha(\omega)$ a complex function of the frequency. Taking the first 3 terms of the Taylor expansion of $\alpha(\omega)$ around $\omega_{0}$ this can be written as

$$
T_{t o t}(\omega) \approx \mathrm{e}^{\mathrm{i} \alpha_{0}+\mathrm{i} \alpha_{1}\left(\omega-\omega_{0}\right)+\mathrm{i} \frac{1}{2} \alpha_{2}\left(\omega-\omega_{0}\right)^{2}}
$$

with $\alpha_{0}=\alpha\left(\omega_{0}\right), \alpha_{1}=\left.\frac{d \alpha}{d \omega}\right|_{\omega 0}$ and $\alpha_{2}=\left.\frac{d^{2} \alpha}{d \omega^{2}}\right|_{\omega 0}$ all complex quantities. This notation will be used in the following section.

\section{A.2.2 Interferometric detection}

A schematic representation of the linear interferometric setup is depicted in Figure A.1. The sample is placed in one of the branches of a MachZehnder interferometer, the input light source is a laser producing femtosecond pulses. By scanning the path length of one of the branches, an interferogram of the pulses is measured. The maximum of this interferogram will be measured when the pulses in the two branches have an exactly equal optical path length. The delay line position for which this occurs depends on the velocities with which the pulses traveled through both branches or equivalently on the difference in optical path length. The maximum of the interferogram will be found therefore at a different delay-line position if the propagation through the sample is fast with respect to when it is slow. This 
way, from the position of the interferogram information on the propagation velocity through the sample is obtained. By comparing the position of the interferograms to an interferogram measured on a reference structure, the group delay can be determined.

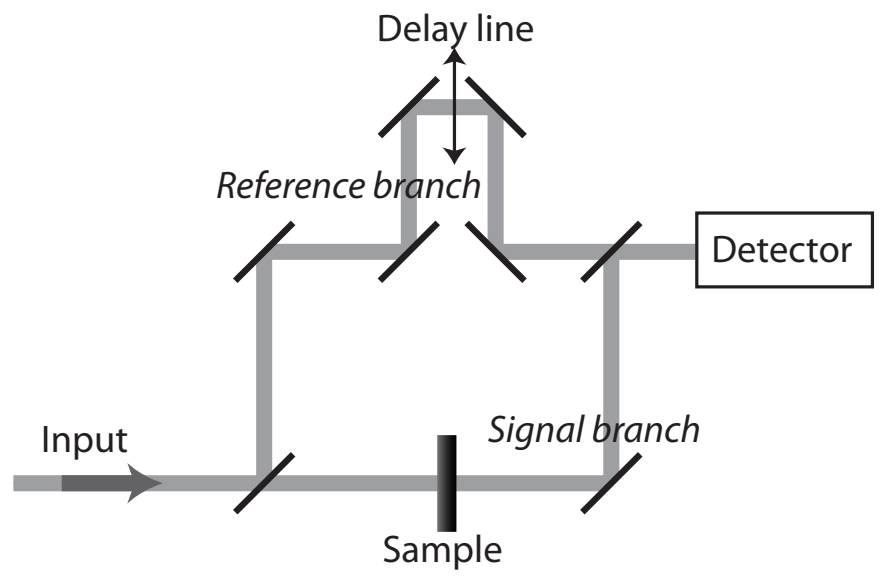

Figure A.1: Schematic showing the interferometric detection setup for the measurement of group delay.

The technique used to measure the group delay makes use of a lock-in detection technique that allows us to determine the full complex interferogram. More detailed technical information of this technique is given in Chapter 3. The detected voltage is given by (see [91] p. 19-22 or [62])

$$
V(\tau)=2 C_{\text {det }} \mathfrak{F}^{-1}\left\{E_{\text {ref }}^{*}(\omega) E_{\text {sig }}(\omega)\right\},
$$

where $C_{d e t}$ is a constant describing the detection efficiency. This expression is valid under the assumption that the integration time of the detection is much longer than the pulse duration. The input signal before the beam splitter in the interferometer is assumed to be a pulse with a Gaussian spectrum and possibly a non-negligible chirp.

$$
A(\omega)=\tilde{A} \mathrm{e}^{\sigma\left(\omega-\omega_{0}\right)^{2}+\mathrm{i} \gamma(\omega)}
$$

where $\gamma(\omega)$ is any real function of $\omega$ describing the phase of the pulse and $\sigma=\frac{1}{\Delta \omega^{2}}$ describes the width of the pulse. The transfer function for the 
sample can be written as,

$$
T_{s}(\omega)=\mathrm{e}^{\mathrm{i} s(\omega)},
$$

with $s(\omega)$ a complex function. The real part of $s(\omega)$ describes the phase evolution while the imaginary part describes the wavelength dependent transmission. In a similar way the propagation over the delay line can be described by

$$
T_{d}(\omega)=\mathrm{e}^{\mathrm{i} \omega \tau} .
$$

The fields interfering at the detector are

$$
E_{r e f}(\omega)=A(\omega) T_{d}(\omega),
$$

that describes the light that propagated through the reference branch and

$$
E_{\text {sig }}(\omega)=A(\omega) T_{s}(\omega)
$$

which describes the light that propagated through the sample. The expression for the detected voltage then becomes (dropping the $\omega$ dependence for sake of simplicity in notation)

$$
\begin{aligned}
V(\tau) & =2 * C_{\operatorname{det}} \mathfrak{F}^{-1}\left\{A^{*} T_{d}^{*} A T_{s}\right\} \\
& =2 * C_{\operatorname{det}} \mathfrak{F}^{-1}\left\{|A|^{2} T_{d}^{*} T_{s}\right\} .
\end{aligned}
$$

A very elegant consequence of using the linear interferogram technique becomes clear now: the complex amplitude of the incoming pulse $A$ appears in the expression for the detected voltage as its absolute value squared $|A|^{2}$. Any chirp or other phase factors that the pulse might have had before it was split in signal and reference branch will therefore no longer play a role in the observation of the group delay.

We will now make some explicit assumptions and evaluate the effect of attenuation in the sample on the observed delay. Writing the combined effect of the transfer functions $T_{d}^{*}$ and $T_{s}$ in one transfer function $T_{t o t}$, and taking the first 3 terms of the Taylor expansion around $\omega_{0}$ :

$$
\begin{aligned}
T_{t o t} & =T_{d}^{*} T_{s} \\
& =\mathrm{e}^{\mathrm{i}\left(\alpha^{\prime}+\mathrm{i} \alpha^{\prime \prime}\right)} \\
& \approx \mathrm{e}^{\mathrm{i} \alpha_{0}+\mathrm{i} \alpha_{1}\left(\omega-\omega_{0}\right)+\mathrm{i} \frac{1}{2} \alpha_{2}\left(\omega-\omega_{0}\right)^{2}} \\
& =\mathrm{e}^{\mathrm{i}\left(s_{0}^{\prime}+\mathrm{i} s_{0}^{\prime \prime}-\omega_{0} \tau\right)+\mathrm{i}\left(s_{1}^{\prime}+\mathrm{i} s_{1}^{\prime \prime}-\tau\right)\left(\omega-\omega_{0}\right)+\mathrm{i} \frac{1}{2}\left(s_{2}^{\prime}+\mathrm{i} s_{2}^{\prime \prime}\right)\left(\omega-\omega_{0}\right)^{2}} .
\end{aligned}
$$


Performing the Fourier transform of Equation A.23 leads to

$$
V(\tau) \propto \frac{\mathrm{e}^{\mathrm{i} \alpha_{0}} \mathrm{e}^{\mathrm{i} \omega_{0} \tau}}{\sqrt{\frac{1}{2} \sigma-\frac{1}{2} \mathrm{i} \alpha_{2}}} \mathrm{e}^{\frac{1}{4}\left(\frac{\mathrm{i}\left(\tau+\alpha_{1}\right)}{2 \sigma+\mathrm{i} \frac{1}{2} \alpha_{2}}\right)^{2}}
$$

This expression can be rewritten using the expressions for $\alpha_{n}$ which leads to a quite involved expression. We are interested only in the factors that determine the position of the envelope. These are determined by the term $\tau_{d}$ if we rewrite the expression in the form

$$
V\left(\tau_{d}\right) \propto \mathrm{e}^{-\xi\left(\tau-\tau_{d}\right)^{2}}
$$

where both $\xi$ and $\tau_{d}$ are real. Then

$$
\begin{aligned}
\tau_{d} & =s_{1}^{\prime}-s_{1}^{\prime \prime} \frac{\operatorname{Im}\left(\left(4 \sigma-s_{2}^{\prime \prime}+\mathrm{i} s_{2}^{\prime}\right)^{2}\right)}{\operatorname{Re}\left(\left(4 \sigma-s_{2}^{\prime \prime}+\mathrm{i} s_{2}^{\prime}+\mathrm{i} b_{2}\right)^{2}\right)} \\
& =s_{1}^{\prime}-s_{1}^{\prime \prime} \frac{\left(4 \sigma-s_{2}^{\prime \prime}\right)\left(s_{2}^{\prime}\right)}{\left(4 \sigma-s_{2}^{\prime \prime}\right)^{2}-\left(s_{2}^{\prime}\right)^{2}} .
\end{aligned}
$$

The factor $s_{1}^{\prime}$ is the group delay introduced by the sample, this is the factor we are interested in for the measurement. The second term describes how the wavelength-dependent transmission of the sample $\left(s_{1}^{\prime \prime}\right)$ leads to an observed delay of the pulse. We can use this expression further on in this thesis to check whether the observed delay has any contribution that originates from attenuation.

\section{A.3 Conclusion}

Equation A.27 allows us to determine the influence of attenuation on the delay of the envelope of the pulse, based on the first 3 terms in the Taylor series of a complex transfer function. Additionally it gives us insight in the way attenuation and group velocity dispersion lead to a change in delay of the envelope of a pulse. 


\section{Bibliography}

[1] T. W. Ebbesen et al. Extraordinary optical transmission through subwavelength hole arrays. Nature 391, 667 (1998).

[2] A. Degiron et al. Optical transmission properties of a single subwavelength aperture in a real metal. Optics Communications 239, 61 (2004).

[3] N. Yamamoto, H. Sugiyama and A. Toda. Cherenkov and transition radiation from thin plate crystals detected in the transmission electron microscope. Proceedings of the Royal Society of London Series AMathematical Physical and Engineering Sciences 452, 2279 (1996).

[4] N. Yamamoto, K. Araya and F. de Abajo. Photon emission from silver particles induced by a high-energy electron beam. Physical Review B 6420, 205419 (2001).

[5] I. Frank and G. V. Radiation of a uniformly moving electron due to its transition from one medium into another. Journal of Physics 9, 353 (1945).

[6] H. Lezec et al. Beaming light from a subwavelength aperture. Science 297, 820 (2002).

[7] S. Kim et al. High-harmonic generation by resonant plasmon field enhancement. Nature 453, 757 (2008).

[8] J. Pendry. Negative refraction makes a perfect lens. Physical Review Letters 85, 3966 (2000).

[9] J. Pendry, D. Schurig and D. Smith. Controlling electromagnetic fields. Science 312, 1780 (2006). 
[10] H. Mertens. Ellipsometry measurements performed with Hans Mertens at the AMOLF institute.

[11] F. Wooten. Optical properties of solids. Academic Press (1972).

[12] L. Novotny and B. Hecht. Principles of Nano-Optics. Cambridge University Press (2006).

[13] H. Reather. Surface Plasmons. Springer-Verlag (1988).

[14] A. Zayats and I. Smolyaninov. Near-field photonics: surface plasmon polaritons and localized surface plasmons. Journal of Optics A-Pure and applied optics 5, S16 (2003).

[15] C. Bohren and D. Huffman. Absorption and scattering of light by small particles. Wiley (1983).

[16] D. Fromm et al. Gap-dependent optical coupling of single "bowtie" nanoantennas resonant in the visible. Nano Letters 4, 957 (2004).

[17] M. Anderson. Locally enhanced raman spectroscopy with an atomic force microscope. Applied Physics Letters 76, 3130 (2000).

[18] A. Sommerfeld. Propagation of waves in wireless telegraphy. Annalen der Physik 333, 665 (1909).

[19] J. Stratton. Electromagnetic Theory. McGraw-Hill book company (1941).

[20] R. Collin. Hertzian dipole radiating over a lossy earth or sea: Some early and late 20th-century controversies. Ieee Antennas and Propagation Magazine 46, 64 (2004).

[21] G. Ford and W. Weber. Electromagnetic interactions of molecules with metal surfaces. Physics Reports 113, 195 (1984).

[22] H. J. Lezec and T. Thio. Diffracted evanescent wave model for enhanced and suppressed optical transmission through subwavelength hole arrays. Optics Express 12, 3629 (2004).

[23] F. J. Garcia-Vidal, S. G. Rodrigo and L. Martin-Moreno. Foundations of the composite diffracted evanescent wave model. Nature Physics $\mathbf{2}$, 790 (2006). 
[24] P. Lalanne and J. P. Hugonin. Interaction between optical nano-objects at metallo-dielectric interfaces. Nature Physics 2, 551 (2006).

[25] H. T. Liu and P. Lalanne. Microscopic theory of the extraordinary optical transmission. Nature 452, 728 (2008).

[26] Y. Gravel and Y. Sheng. Rigorous solution for the transient surface plasmon polariton launched by subwavelength slit scattering. Opt. Express 16, 21903 (2008).

[27] P. M. Morse and H. Feshbach. Methods of Theoretical Physics - Part 1. Feshbach Publishing (1953).

[28] K. J. K. Koerkamp et al. Strong influence of hole shape on extraordinary transmission through periodic arrays of subwavelength holes. Physical Review Letters 92, 183901 (2004).

[29] R. Gordon et al. Strong polarization in the optical transmission through elliptical nanohole arrays. Physical Review Letters 92, 037401 (2004).

[30] H. Bethe. Theory of diffraction by small holes. Physical Review 66, 163 (1944).

[31] C. Bouwkamp. On the diffraction of electromagnetic waves by small circular disks and holes. Philips Reasearch Reports 5, 401 (1950).

[32] D. Griffiths. Introduction to Electrodynamics. Prentics -Hall (1989).

[33] R. Gordon and A. G. Brolo. Increased cut-off wavelength for a subwavelength hole in a real metal. Optics Express 13, 1933 (2005).

[34] E. N. Economou. Surface plasmons in thin films. Phys. Rev. 182, 539 (1969).

[35] S. Collin, F. Pardo and J.-L. Pelouard. Waveguiding in nanoscale metallic apertures. Optics Express 15, 4310 (2007).

[36] K. L. van der Molen et al. Role of shape and localized resonances in extraordinary transmission through periodic arrays of subwavelength holes: Experiment and theory. Physical Review B 72, 045421 (2005).

[37] F. J. Garcia-Vidal et al. Transmission of light through a single rectangular hole. Physical Review Letters 95, 103901 (2005). 
[38] F. J. Garcia-Vidal et al. Transmission of light through a single rectangular hole in a real metal. Physical Review B 74, 153411 (2006).

[39] A. K. Sarychev et al. Resonance transmittance through a metal film with subwavelength holes. Ieee Journal Of Quantum Electronics 38, 956 (2002).

[40] L. Yin et al. Surface plasmons at single nanoholes in au films. Applied Physics Letters 85, 467 (2004).

[41] A.-L. Baudrion et al. Coupling efficiency of light to surface plasmon polariton for single subwavelength holes in a gold film. Opt. Express 16, 3420 (2008).

[42] E. Popov et al. Surface plasmon excitation on a single subwavelength hole in a metallic sheet. Applied Optics 44, 2332 (2005).

[43] S. Chang, S. Gray and G. Schatz. Surface plasmon generation and light transmission by isolated nanoholes and arrays of nanoholes in thin metal films. Optics Express 13, 3150 (2005).

[44] J. Alegret, P. Johansson and M. Kall. Green's tensor calculations of plasmon resonances of single holes and hole pairs in thin gold films. New Journal of Pysics 10, 105004 (2008).

[45] E. Popov et al. Theory of light transmission through subwavelength periodic hole arrays. Physical Review B 62, 16100 (2000).

[46] L. Martin-Moreno et al. Theory of extraordinary optical transmission through subwavelength hole arrays. Physical Review Letters 86, 1114 (2001).

[47] Q. Cao and P. Lalanne. Negative role of surface plasmons in the transmission of metallic gratings with very narrow slits. Physical Review Letters 88, 057403 (2002).

[48] W. Barnes et al. Surface plasmon polaritons and their role in the enhanced transmission of light through periodic arrays of subwavelength holes in a metal film. Physical Review Letters 92, 107401 (2004).

[49] U. Fano. Effects of configuration interaction on intensities and phase shifts. Phys. Rev. 124, 1866 (1961). 
[50] C. Genet, M. van Exter and J. Woerdman. Fano-type interpretation of red shifts and red tails in hole array transmission spectra. Optics Communications 225, 331 (2003).

[51] H. F. Ghaemi et al. Surface plasmons enhance optical transmission through subwavelength holes. Physical Review B 58, 6779 (1998).

[52] D. E. Grupp et al. Crucial role of metal surface in enhanced transmission through subwavelength apertures. Applied Physics Letters 77, 1569 (2000).

[53] J. Pendry, L. Martin-Moreno and F. Garcia-Vidal. Mimicking surface plasmons with structured surfaces. Science 305, 847 (2004).

[54] A. Dogariu et al. Delay in light transmission through small apertures. Optics Letters 26, 450 (2001).

[55] A. Dogariu et al. Optical pulse propagation through metallic nanoapertures. Applied Physics B-Lasers And Optics 74, S69 (2002).

[56] R. Muller, C. Ropers and C. Lienau. Femtosecond light pulse propagation through metallic nanohole arrays: The role of the dielectric substrate. Optics Express 12, 5067 (2004).

[57] G. A. Wurtz, R. Pollard and A. V. Zayats. Optical bistability in nonlinear surface-plasmon polaritonic crystals. Physical Review Letters 97, 057402 (2006).

[58] M. Airola, Y. Liu and S. Blair. Second-harmonic generation from an array of sub-wavelength metal apertures. Journal Of Optics A-Pure And Applied Optics 7, S118 (2005).

[59] R. Boyd. Nonlinear Optics. Academic Press (2003).

[60] J. I. Dadap et al. Second-harmonic rayleigh scattering from a sphere of centrosymmetric material. Phys. Rev. Lett. 83, 4045 (1999).

[61] J. A. H. van Nieuwstadt et al. Strong modification of the nonlinear optical response of metallic subwavelength hole arrays. Physical Review Letters 97, 146102 (2006). 
[62] M. Sandtke et al. Novel instrument for surface plasmon polariton tracking in space and time. Review of scientific instruments 79, 013704 (2008).

[63] R. Moerland. FDTD calculations performed by Robert Moerland at the University of Twente.

[64] A. Mary et al. Theory of light transmission through an array of rectangular holes. Physical Review B 76, 195414 (2007).

[65] V. Ginzburg and I. Frank. ZhETF 16, 15 (1946).

[66] V. Ginzburg and V. Tsytovich. Transition radiation and transition scattering. Adam Hilger (1990).

[67] F. J. García de Abajo and A. Howie. Relativistic electron energy loss and electron-induced photon emission in inhomogeneous dielectrics. Phys. Rev. Lett. 80, 5180 (1998).

[68] F. J. García de Abajo and A. Howie. Retarded field calculation of electron energy loss in inhomogeneous dielectrics. Phys. rev. B 65, 115418 (2002).

[69] E. J. R. Vesseur et al. Surface plasmon polariton modes in a singlecrystal au nanoresonator fabricated using focused-ion-beam milling. Applied Physics Letters 92, 083110 (2008).

[70] E. Betzig et al. Breaking the diffraction barrier: Optical microscopy on a nanometric scale. Science 251, 1468 (1991).

[71] S. W. Hell. Far-field optical nanoscopy. Science 316, 1153 (2007).

[72] R. H. Ritchie. Plasma losses by fast electrons in thin films. Phys. Rev. 106, 874 (1957).

[73] A. Kubo et al. Femtosecond imaging of surface plasmon dynamics in a nanostructured silver film. Nano Letters 5, 1123 (2005).

[74] E. J. R. Vesseur et al. Direct observation of plasmonic modes in au nanowires using high-resolution cathodoluminescence spectroscopy. Nano Letters 7, 2843 (2007). 
[75] J. Nelayah et al. Mapping surface plasmons on a single metallic nanoparticle. Nature Physics 3, 348 (2007).

[76] J. T. van Wijngaarden et al. Direct imaging of propagation and damping of near-resonance surface plasmon polaritons using cathodoluminescence spectroscopy. Applied Physics Letters 88, 221111 (2006).

[77] M. Bashevoy et al. Generation of traveling surface plasmon waves by free-electron impact. Nano Letters 6, 1113 (2006).

[78] L. Ozawa. Cathodoluminescence. Kodansha (1990).

[79] F. J. Garcia de Abajo. unpublished .

[80] N. Yamamoto and T. Suzuki. Conversion of surface plasmon polaritons to light by a surface step. Applied Physics Letters 93, 093114 (2008).

[81] M. Kuttge. Calculated by Martin Kuttge at the AMOLF institute.

[82] C. Genet and T. W. Ebbesen. Light in tiny holes. Nature 445, 39 (2007).

[83] T. Sondergaard. Modeling of plasmonic nanostructures: Green's function integral equation methods. Physica Status Solidi B-Basic Solid State Physics 244, 3448 (2007).

[84] H. Fischer et al. Characterization of the polarization sensitivity anisotropy of a near-field probe using phase measurements. Journal of Microscopy-Oxford 230, 27 (2008).

[85] B. Sepulveda et al. Shape effects in the localized surface plasmon resonance of single nanoholes in thin metal films. Optix Express 16, 5609 (2008).

[86] E. Prodan et al. A hybridization model for the plasmon response of complex nanostructures. Science 302, 419 (2003).

[87] Y. Alaverdyan et al. Optical antennas based on coupled nanoholes in thin metal films. Nature Physics 3, 884 (2007).

[88] F. Przybilla, C. Genet and T. W. Ebbesen. Enhanced transmission through penrose subwavelength hole arrays. Applied Physics Letters 89, 121115 (2006). 
[89] M. Soljacic and J. D. Joannopoulos. Enhancement of nonlinear effects using photonic crystals. Nature Materials 3, 211 (2004).

[90] L. Brillouin. Wave propagation and group velocity. Academic Press (1960).

[91] R. Engelen. Ultrafast investigations of slow light in photonic crystal structures. Ph.D. thesis, FOM Institute for Atomic and Molecular Physics (AMOLF) (2008). 


\section{Samenvatting}

Het onderzoeksgebied van de nano-optica is een bruisend vakgebied, dat volop in ontwikkeling is. Een van de doelen van het vakgebied is om controle over het gedrag van licht te krijgen, op een schaal kleiner dan de golflengte. Nano-plasmonische structuren zijn op dit moment de meest veel belovende materialen om dit te bereiken.

In 1998 ontdekten Ebbesen en zijn collega's dat de transmissie van licht door roosters van gaten met een afmeting kleiner dan de golflengte van licht zeer efficint kan zijn. Deze kan zelfs zo efficint zijn, dat de transmissie genormaliseerd door de open fractie van de structuur groter is dan 1. Deze ontdekking was een grote verassing, want tot op dat moment werd de transmissie van licht door sub golflengte-gaten als zeer inefficint beschouwd. Na deze ontdekking zijn subgolflengte-gaten een van de meest populaire nanoplasmonische structuren geworden. In dit proefschrift worden de dynamische en de locale eigenschappen van de interactie van licht met subgolflengtegaten bestudeerd.

De dynamische eigenschappen van de interactie van licht met subgolflengte-gaten is bestudeerd door de relatie tussen de vorm van gaten en de groepsvertraging van lichttransmissie door roosters van subgolflengte-gaten te meten. Hiervoor is een interferometrische opstelling gebouwd waarmee de vertraging van femtoseconde pulsen door de roosters kan worden gemeten. De metingen laten zien dat de tijdsvertraging maximaal is als de golflengte van het licht zo gekozen wordt, dat deze overeenkomt met de golflengte waarop de transmissie afkapt - de zogenaamde afkapfrequentie. Ook is onderzocht hoe de gemeten tijdvertraging zich verhoud tot de hoeveelheid tweede harmonische die gegenereerd wordt als een korte intense lichtpuls door het rooster van gaten beweegt. Het blijkt dat een hogere pulsvertraging gepaard gaat met een hogere tweede harmonische generatie. Dit betekent dat de afkapfrequentie gebruikt kan worden om niet-lineare 
processen te versterken.

De locale eigenschappen van de interactie van licht met subgolflengtegaten worden in dit proefschrift onderzocht met een speciale techniek die gebruik maakt van de transitiestraling. Deze straling ontstaat wanneer een elektron door het oppervlak tussen twee materialen met een verschillende brekingsindex beweegt. Met een elektronenmicroscoop is het mogelijk een gefocusseerde bundel elektronen op iedere gewenste positie op een object te richten. De transitiestraling die gegenereerd wordt, is afhankelijk van de positie van de elektronenbundel op de nano-plasmonische structuur.

Door de transitiestraling te verzamelen met een parabolische spiegel en dit licht te meten met een spectrometer kunnen we een 'afbeelding' van de nano-plasmonische structuur maken door op een raster van punten te meten. De resolutie wordt door de breedte van de gefocusseerde elektronenbundel bepaald. Deze techniek geeft dus inzicht in de optische eigenschappen van structuren met een resolutie die veel kleiner is dan de golflengte van het licht.

Met transitiestraling-microscopie zijn metingen verricht aan een enkel rond gat. De resultaten laten zien dat de transitiestraling toeneemt als de elektronenbundel dichter bij de rand van het gat komt. Bovenop deze toename is een oscillerende intensiteit als functie van afstand te zien, die afhankelijk is van de golflengte van het licht. We modeleren dit systeem door het gat te modeleren als een elektrische dipool die aangedreven wordt door het elektromagnetische veld op het oppervlak. Het verzamelde licht bestaat uit het licht dat direct gegenereerd wordt op de positie van de elektronenbundel en licht dat aan het gat verstrooid wordt. Op basis van het model kunnen we een schatting maken van de effectieve polariseerbaarheid van het gat.

Door naar rechthoekige gaten te kijken met de transitiestraling-microscopietechniek, ontdekten we dat er langs de rechte zijden van het gat maxima waarneembaar zijn. Deze maxima hebben we nader onderzocht door systematisch de gatvorm van rechthoekige gaten te variren en naar het gemeten spectrum te kijken. In dit spectrum is slechts n piek te zien, die verschuift als de gatvorm veranderd wordt. Hierbij spelen de afmetingen van beide zijden van het gat een rol. Op grond van de gemeten resultaten vermoeden wij dat er een relatie bestaat tussen het gemeten licht en de afkapfrequentie van het gat.

De polarisatie van het verzamelde licht kan worden geanalyseerd door 
in de opstelling een polarisatiefilter te plaatsen. In een meting aan een rechthoekig gat voor een aantal verschillende hoeken van de analysator observeren wij een duidelijke polarisatie-afhankelijkheid die van plaats tot plaats verschilt. Door aan te nemen dat het licht dat dicht bij het gat gemaakt wordt vooral recht omhoog van het oppervlak verstrooit, kunnen we de gemeten polarisatie aan de verstrooide polarisatie verbinden. Wij observeren dan dat de polarisatie voornamelijk loodrecht op de assen van het gat staat. Dit komt goed overeen met wat verwacht kan worden voor de verstrooiing van oppervlakteplasmonen.

In een structuur met meerdere gaten speelt ook de verstrooiing aan en tussen meerdere gaten een rol. We hebben een experiment uitgevoerd waarbij de afstand tussen een drietal rechthoekige gaten wordt gevarieerd en bekeken wordt hoe het spectrum in de maxima direct langs de zijden van het gat varieert als functie van de afstand. We vinden een opmerkelijk grote invloed van deze afstand ondanks dat de patronen die we observeren op het oog eigenlijk nauwelijks verschillen. 


\section{Dankwoord}

\begin{tabular}{|c|c|c|c|}
\hline & Miller & $\begin{array}{l}\text { Tref } \\
\text { t \& Pigno }\end{array}$ & \\
\hline Kobus & Mattec & Hinco & Dries \\
\hline Marko & Aron & Tobias & Jean \\
\hline Rob & Hans & Chris & Gijs \\
\hline \multirow[t]{2}{*}{ Femius } & René & Martin & Ernst-Jan \\
\hline & \multicolumn{2}{|c|}{ Rutger } & \\
\hline \multirow{3}{*}{ Jelle } & $\mathrm{Pa}$ & a $\mathrm{Ma}$ & \\
\hline & \multicolumn{2}{|r|}{ Maarten } & Marc Q \\
\hline & & Lou & \\
\hline
\end{tabular}

Andreas Frans Robert

Maaike Matthew

Jeroen Casper Marjolijn

Fenneke Titus Dries

Janne 